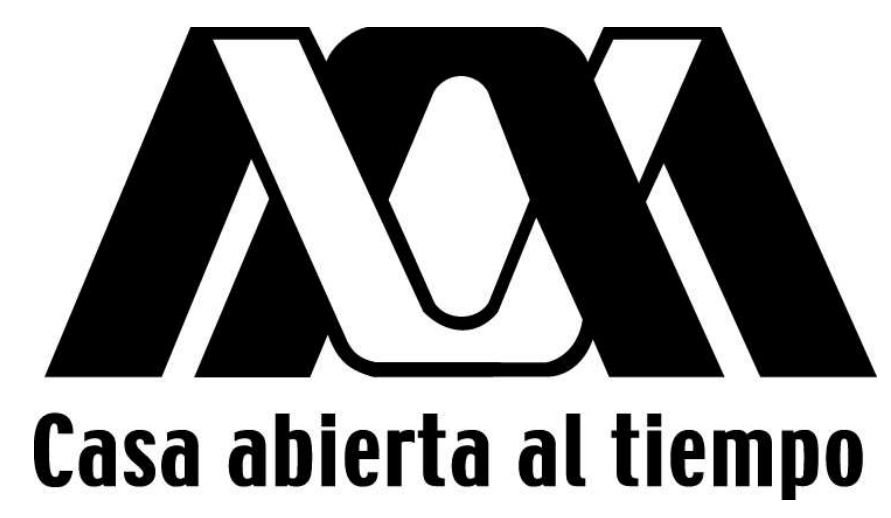

\title{
Tratamiento teórico de efectos moleculares en el poder de frenamiento de iones en materia condensada
}

\author{
Tesis que presenta:
}

M. en C. Remigio Cabrera Trujillo

para obtener el grado de Doctor en Ciencias

Departamento de Física,

División de Ciencias Básicas,

Universidad Autónoma Metropolitana-Iztapalapa,

México

Abril, 1998 
Dedicado a las dos mujeres de mi vida.

Mi madre y mi amada esposa

por siempre estar ahí por mí

$y$ darme fuerzas. 


\section{Agradecimientos}

Esta tesis es el fruto de mis estudios de doctorado en dos lugares muy especiales donde lo he llevado a cabo: el Departamento de Física de la UAM-I y el Departamento de Química de la Universidad de Odense, Dinamarca. Quiero agradecer a toda esa gente entre la que me rodeé y con la que compartí momentos agradables enseñándome y discutiendo cosas. En especial quiero agradecer a mi asesor el Dr. Salvador Cruz Jiménez por siempre motivarme y responder sin descanso a mis preguntas una y otra vez. De la misma forma quiero agradecer a mis asesores externos, el Dr. Jens Oddershede en Dinamarca y el Dr. John R. Sabin de la Universidad de Florida en E.U.A. por haberme tolerado y mostrado otra forma de ver el mundo de la física, muchas gracias. También quiero agradecer a un sinúmero de personas entre quienes llegué a tener una muy bonita amistad, en especial a Torben Rasmunssen, mi compañero de oficina en Dinamarca, que sin su cálida amistad, el clima frio me hubiera hecho más efecto y por lo que seguramente hubiera dedicado más tiempo a mi trabajo; pero que ha sido un excelente amigo. Sin querer olvidar a los demás quiero agradecer a Sheela Kirphekar, Stephan Sauer, Trond Saue, Luuk Visscher, Thomas Enevoldsen, y de manera muy especial a H. A. Jørgens sin quien por su experiencia en DALTON, me hubiera sido difícil llevar a cabo mi trabajo computacional en Dinamarca, y a mucha otra gente tanto aquí en México como en Dinamarca que me ayudaron durante mi trabajo. Aquí en México quiero agradecer a mi compañera de oficina, Rebeca Sosa, por haberme soportado y tolerar mis regaños, así como a todos los miembros del laboratorio donde tengo mi mazmorra.

De igaul manera deceo agradecer a los sinodales, Dr. Marcelo Lozada Cassou, Dr. John R. Sabin, Dra. María del Carmen Cisneros Gudiño, Dr. Eleuterio Castaño Tostado quienes con una gran paciencia y entusiasmo han ido corrigiendo y comentando este trabajo, haciendolo aun más valioso para mi. 
Por otra parte quiero agradecer al CONACyT el apoyo económico prestado para el desarrollo de mi doctorado a través de la beca crédito Registro 89450, así como del proyecto de investigación No. 1405-E9207, durante mi estancia en Dinamarca. De igual manera agradesco al gobierno Danés, principalmente a la "Danish Research Academy" y a la "Danish Natural Science Research Council" por su valioso apoyo en mi estancia en Dinamarca.

Y finalmente agradezco a aquella gente, que no he nombrado y sin ser menos han contribuido en mi desarrollo.

Muchas gracias a todos ellos!.

R. C. T. 


\section{Contenido}

Agradecimientos $\quad$ v

1 Introducción 1

1.1 Propósito de este trabajo . . . . . . . . . . . . . . . . 3

2 Poder de frenamiento molecular 5

2.1 Resumen . . . . . . . . . . . . . . . . . 5

2.2 Introducción . . . . . . . . . . . . . . . . . . 5

2.3 Teoría cinética . . . . . . . . . . . . . . . . . 6

2.4 Modelo OLPA/FSGO . . . . . . . . . . . . . . . . . 10

2.4.1 Aproximación de Plasma Local (LPA) . . . . . . . . . . . . 10

2.4.2 Aproximación de plasma local por orbital (OLPA) . . . . . . 11

2.5 Orbitales moleculares FSGO . . . . . . . . . . . . . . . . . 13

2.6 Implementación al poder de frenamiento . . . . . . . . . . . . . . . 14

2.7 Análisis de los resultados . . . . . . . . . . . . . . . . . 16

2.8 Conclusiones. . . . . . . . . . . . . . . . . . . . . . . . . . 23

3 Teoría de Bethe: proyectiles con estructura 25

3.1 Resumen ........................ 25

3.2 Introducción . . . . . . . . . . . . . . . . . . . 25

3.3 El poder de frenamiento: Desarrollo General . . . . . . . . . . . . . 27

3.3.1 La aproximación de Bethe . . . . . . . . . . . . . 33

3.4 Criterio adiabático para $N_{1}(v) \ldots \ldots \ldots$. . . . . . . . 34

3.5 Expresión analítica para $S_{e}(v) \ldots \ldots . \ldots . \ldots . \ldots$

3.6 Análisis y discusión . . . . . . . . . . . . . . . . . . . . . . . . . . . 39

3.6.1 La precisión de la suposición adiabática . . . . . . . . . . 41 
viii

3.6.2 Comparación con el experimento . . . . . . . . . . . 43

3.7 Conclusiones . . . . . . . . . . . . . . . . . . . . . 48

4 BSR en la aproximación RPA $\quad 49$

4.1 Resumen ....................... . . 49

4.2 Introducción . . . . . . . . . . . . . . . . . . . . 49

4.3 Las ecuaciones de Hartree-Fock . . . . . . . . . . . . . . . . . . 51

4.4 Modelo RPA . . . . . . . . . . . . . . . . . . . . . . 55

4.5 La regla de suma de Bethe en RPA . . . . . . . . . . . . . 58

$\begin{array}{lll}5 & \text { BSR en un campo EM } & 63\end{array}$

5.1 Resumen ....................... . . 63

5.2 Introducción . . . . . . . . . . . . . . . 63

5.3 Hamiltoniano para un campo electromagnético . . . . . . . . 65

5.4 Regla de suma de Bethe . . . . . . . . . . . . . . . 67

5.5 Análisis perturbativo . . . . . . . . . . . . . . . 69

$\begin{array}{lll}6 & \text { Conclusiones generales } & 75\end{array}$

$\begin{array}{ll}\text { A Teoría de Bethe estandard } & 79\end{array}$

B Simplificación algebraica de $S_{e}(v) \quad 83$

$\begin{array}{lll}\text { C } & \text { Tensiones de Oscilador Generalizado } & 87\end{array}$

$\begin{array}{ll}\text { D Regla de suma parcial } & 91\end{array}$

$\begin{array}{lll}\text { E Integración de } S_{e} \text { para q pequeñas } & 93\end{array}$

$\begin{array}{ll}\text { Bibliografía } & 95\end{array}$

Artículo I:

R. Cabrera-Trujillo, S. A. Cruz and J. Soullard.

"Bond stopping cross sections for protons incidents on molecular targets within the OLPA/FSGO implementation of the kinetic theory."

Nucl. Instr. and Meth. B93, pp. 166 to 174 (1994).

Artículo II: 
R. Cabrera-Trujillo, S. A. Cruz, Jens Oddershede and John R. Sabin.

"Bethe theory of stopping incorporating electronic excitations of partially stripped projectiles,"

Phys. Rev. A 55, pp. 2864 to 2872 (1997).

\section{Artículo III:}

R. Cabrera-Trujillo, John R. Sabin, Y. Öhrn and J. Oddershede, "Oscillator Strength Sum Rules with an External Electromagnetic Field",

Phys. Rev. A 57, pp. 3115 to 3118 (1998).

Artículo IV:

R. Cabrera-Trujillo, John R. Sabin, J. Oddershede, and Stephan P. A. Sauer, "The Bethe sum rule and basis set selection in the calculation of Generalized Oscillator Strengths",

Advances in Quantum Chemistry 35, 175 (1999). 


\title{
Capítulo 1
}

\section{Introducción}

\author{
"Somos una civilización científica. \\ Eso significa una civilización en la que el \\ saber y su integridad son factores cruciales. \\ Ciencia no es más que una palabra \\ latina que significa conocimiento ... \\ Nuestro destino es el conocimiento." \\ Jacob Bronowski, \\ "The ascent of Men", \\ Little Brown, Boston 1973
}

El aspecto teórico de la interacción de particulas cargadas con materia ha atraido la atención de físicos notables desde hace ya varias décadas [1]-[9]. De hecho, las investigaciones teóricas y experimentales de este problema han jugado un papel muy importante en el desarrollo de la física moderna. Durante la década de 1890, resultó claro que los átomos estaban constituidos por partículas aún más pequeñas. En 1897 Sir Joseph John Thomson [1] estableció la existencia examinando las propiedades de las partículas involucradas en descargas eléctricas, llamados electrones, los cuales forman la unidad básica de la electricidad negativa. Al mismo tiempo que Thomson investigaba las propiedades de los electrones, otro descubrimiento de gran importancia era llevado a cabo por el físico Frances Henri Becquerel y posteriormente por Pierre y Marie Curie. Ellos encontraron que ciertos materiales emitían radiaciones de tipo desconocido, que eventualmente fueron clasificadas en rayos $\alpha, \beta$ y $\gamma$. 
Para 1900 ya se había establecido que todos los átomos en su estado neutro contienen electrones. Con esto, J. J. Thomson propuso un modelo físico del átomo conocido como "pudín de pasas" en el cual el «pudín》 positivo tenía pasas negativas, los electrones, de tal manera que hicieran neutro al conjunto.

En 1911 el profesor Ernest Rutherford [2], quien había sido discípulo de Thomson, y dos de sus estudiantes, Hans Geiger y Ernest Marsden [3, 4], efectuaron cierto número de experimentos sobre la dispersión de partículas $\alpha$ por una delgada hoja de oro. Rutherford encontró que era capaz de interpretar los resultados experimentales, solo si la masa de un átomo estuviera concentrada casi completamente en una región central de carga positiva: el núcleo atómico.

La manera en la que los rayos $\alpha$ y $\beta$ son influenciados al pasar a través de la materia da una gran cantidad de información, en particular sobre las transmutaciones nucleares. Los detectores de trayectorias de partículas (cámaras de niebla, cámaras de burbujas, emulsiones nucleares, etc. ) han sido responsables directamente de la identificación de muchas de las partículas elementales conocidas. Los detectores de partículas cargadas en general han sido fundamentales en la recopilación de muchos de los resultados experimentales en física de altas, medias y bajas energías. Muchas de las investigaciones en astrofísica, física nuclear, física atómica, física molecular, biofísica y muchos otros campos de investigación en técnicas experimentales que utilizando radiación de altas energías, requieren el conocimiento teórico respecto a la interacción entre la radiación y la materia.

Si bien el estudio de la pérdida de energía de iones al penetrar la materia es de caracter básico, su implementación en aplicaciones tecnológicas no se hizo esperar. El caso de la implantación de iones por bombardeo es un buén ejemplo de una aplicación útil en protección por radiación, radioterapia y radiobiología [10]-[13], por mencionar tan sólo algunos ejemplos. Otra aplicación se da en la industria metalúrgica puesto que con la incorporación de cierto tipo de átomos en dosis y profundidades apropiadas se modifica tanto la dureza como la resistencia a la corrosión de algunas superficies metálicas [14] en forma notable. En la industria electrónica, por ejemplo es posible limpiar una superficie removiendo capas atómicas por bombardeo ionico, ó tambien se puede alterar la topología superficial para crear patrones útiles en la fabricación de microcircuitos. 


\section{$1.1 \quad$ Propósito de este trabajo}

En base a lo anterior, podemos concluir que es imperativo conocer con profundidad los mecanismos mediante los cuales un ion pierde energía al penetrar en diversos materiales para poder contar con capacidad predictiva sobre el daño y modificación que estos sufren al verse afectados por haces de proyectiles, con o sin estructura.

Es en este campo que se presenta esta tesis. Se presentan resultados originales y novedosos que se han desarrollado en relación con el papel que juega la estructura electrónica del proyectil, así como los efectos moleculares del blanco en la pérdida de energía de los iones que penetran materiales compuestos.

Con el objeto de analizar el efecto que tiene la estructura de blancos moleculares en la pérdida de energía, en el capítulo 2 se estudia el frenamiento de protones incidentes sobre moléculas cuyos elementos constitutivos (enlaces, carozos y pares solitarios) se describen mediante orbitales localizados representados por funciones gaussianas esféricas flotantes (FSGO). Dado que la densidad electrónica de la molécula está bién representada por este tipo de orbitales, el tratamiento de la pérdida de energía se lleva a cabo mediante la teoría cinética [15] dentro de la aproximación de plasma local para orbitales (OLPA) [16]. Mediante este tratamiento se obtiene la contribución de carozos, enlaces y pares solitarios a la pérdida de energía [17] y se comparan las predicciones teóricas con el experimento.

En el capítulo 3 se presenta una extensión de la teoría de Bethe para el análisis cuántico de la pérdida de energía para proyectiles con estructura electrónica [18]. Para dar un carácter autocontenido a la tesis, se ha incluido una breve descripción del modelo estandard de Bethe para proyectiles sin estructura electrónica (desnudos) en el Apéndice A. Dado que se considera la estructura del proyectil se encuentra por primera vez la contribución adicional a la pérdida de energía debido a las excitaciones e ionizaciones existentes en la estructura electrónica del proyectil. Como consecuencia de los procesos de pérdida y captura de electrones en colisiones sucesivas, el número promedio de electrones en el proyectil es función de la velocidad de éste último. Para tomar en cuenta el número promedio de electrones retenidos por el proyectil en función de su velocidad, se propone la utilización del criterio adiabático de Bohr dentro del modelo de Thomas-Fermi, obteniéndose de manera análitica una expresión de la "carga efectiva" del proyectil. 
Aún en el tratamiento formal a la Bethe para el caso de blancos atómicos y moleculares es fundamental el cálculo de las Tensiones de Oscilador Generalizado (GOS) para la descripción correcta de las excitaciones e ionizaciones del blanco en el proceso de transferencia de energía. Debido a las dificultades computacionales en el cálculo del espectro completo de excitaciones del blanco, generalmente se recurre a metodos aproximados como lo es la Aproximación de Fase Aleatoria (RPA).

En el capítulo 4 se muestra explícitamente que, dada una base completa, la RPA cumple la regla de suma de Bethe, lo cuál es condición necesaria para que la RPA resulte adecuada para el cálculo de los GOS y llevar a una buena descripción para el proceso de pérdida de energía [19]. En caso de no contar con una base completa, el cumplimiento de la Regla de Suma de Bethe dentro de la RPA, constituye una medida de la calidad de la base.

El uso de la RPA para el caso de blancos atómicos o moleculares puede emplearse en el tratamiento formal del frenamiento de proyectiles con estructura que inciden sobre blancos moleculares. Desde luego, el cálculo es más complicado que el mostrado en el capítulo 4 y es aún motivo de investigación.

Dado que el cumplimiento de la regla de suma de Bethe es fundamental para asegurar una descripción correcta de la estructura atómica o molecular de un sistema y en consecuencia, para considerar el espectro completo de las excitaciones del blanco, en el capítulo 5 se presenta el tratamiento exacto de dicha regla de suma cuando el sistema se encuentra sometido a un campo electromagnético externo [20]. Esto es importante en el caso, por ejemplo, de aceleración de protones en ráfagas solares en donde se generan campos electromagnéticos super-intensos y existen colisiones con átomos y moleculas en la atmósfera estelar [21].

Finalmente en el capítulo 6 se presentan las conclusiones generales, discutiéndose los aspectos bajo estudio actual y futuro, así como las consecuencias y limitaciones de los resultados y tratamientos presentados aquí. 


\section{Capítulo 2}

\section{Efectos del enlace químico en el poder de frenamiento}

\section{$2.1 \quad$ Resumen}

En éste capítulo nos concentraremos en describir un modelo basado en la teoría cinética de frenamiento junto con la aproximación de plasma local para orbitales (OLPA) para obtener un tratamiento del efecto de los enlaces químicos en el poder de frenamiento en blancos moleculares. El modelo consiste en un enfoque autoconsistente basado en la implementación de los Orbitales Gaussianos Esfericos Flotantes (FSGO) para representar los orbitales moleculares, lo cual nos permite explorar las capacidades de la teoría de OLPA implementada dentro del marco de la teoría cinética, pero sin necesitar recurrir a información externa alguna sobre los parámetros, ya sean teóricos o experimentales.

\subsection{Introducción}

Cuando el blanco sobre el que incide un proyectil tiene estructura compleja ( e.g. moléculas, polímeros, etc. ) normalmente se recure a la regla de Bragg [22] para evaluar la sección de frenamiento electrónico, la cual en su forma más simple nos dice que la sección de frenamiento total es la suma de la contribución individual de cada elemento, i.e.

$$
S_{e_{\text {molecula }}}(v)=\sum_{i} n_{i} S_{e, i}(v)
$$


donde $n_{i}$ es el número de átomos del elemento $i$ en la molécula y $S_{e, i}$ es la sección de frenamiento electrónico del elemento $i$. Esta expresión implica que la contribución al enlace químico de los átomos que conforman la molécula es despreciable. Sin embargo, recientemente se ha encontrado evidencia experimental en la que las características de la estructura molecular pueden tener una fuerte influencia sobre la sección de frenamiento $S_{e}$. Recientemente Thwaites [10] ha reportado un análisis completo de ésta evidencia experimental y sobre el efecto que la agregación tiene sobre $S_{e}$ al considerar blancos moleculares, concluyendo que la regla de aditividad de Bragg es inadecuada para explicar el comportamiento experimental de $S_{e}$, principalmente en la región en que las velocidades del proyectil son bajas, e.g, alrededor del máximo de la curva de frenamiento.

Dada la enorme importancia que ésto tiene en el desarrollo de nuevos materiales sintéticos, así como de su uso en el área médica, es necesario conocer más precisamente el poder de frenamiento [23]-[26]; en particular en radioterapia, donde el medio de interés es la célula viva o más especificamente, componentes de la célula que juegan un papel vital en sus capacidades proliferativas o en el tratamiento de tumores cancerígenos mediante haces de protones [27].

Sin embargo, desde el punto de vista teórico, sólo han existido algunos esfuerzos aislados dirigidos al estudio del efecto que el enlace químico tiene sobre la sección de frenamiento electrónico $S_{e}$.

\subsection{Teoría cinética}

El estudio de la pérdida de energía por procesos inelásticos considera en general que la velocidad del proyectil es mayor que la velocidad orbital de los electrones del blanco (átomos, moléculas o electrones), puesto que normalmente se considera que la interacción es de tipo perturbativo, y se supone que los electrones del blanco están en reposo respecto al proyectil. Sin embargo, si los electrones del medio de frenamiento están en equilibrio térmico, el proyectil se frena hasta que su energía cinética ha alcanzado el valor de equilibrio. Esto significa que si el proyectil es más pesado que los electrones del medio de frenamiento, el frenamiento puede continuar hasta velocidades del proyectil muy por debajo de la velocidad térmica de los electrones del blanco. Con lo cual, existe una severa limitación al despreciar el movimiento 
interno de los electrones del blanco.

Esto es particularmente importante para el estudio de la pérdida de energía de iones, puesto que las correcciones por capas son importantes para bajas velocidades.

Para llevar a cabo una descripción general, describiremos escencialmente el tratameinto de P. Sigmund [15] y en el cual, denominemos al proyectil partícula 1 y a los electrones del blanco, partícula 2. Con esto en mente, consideremos - en el sistema de laboratorio $C_{L^{-}}$un proyectil de masa $m_{1}$ y de carga $Z_{1}$ penetrando con una velocidad no relativista $\mathbf{v}_{1}$ en un medio infinito homogéneo compuesto de partículas de masa $m_{2}$ moviéndose con una distribución de velocidades $f\left(\mathbf{v}_{2}\right)$ tal que

$$
\int f\left(\mathbf{v}_{2}\right) d^{3} v_{2}=1
$$

y consideremos que el proyectil interacciona con el medio via colisiones binarias. Despues de la colisión, las velocidades del proyectil y de los electrones del medio serán $\mathbf{v}_{1}^{\prime}$ y $\mathbf{v}_{2}^{\prime}$ respectivamente.

En el centro de masa, $C_{M}$, un electrón del blanco tiene las velocidades

$$
\begin{aligned}
& \mathbf{u}_{2}=\mathbf{v}_{2}-\mathbf{V} \\
& \mathbf{u}_{2}^{\prime}=\mathbf{v}_{2}^{\prime}-\mathbf{V}
\end{aligned}
$$

para antes y despues de la colisión respectivamente y donde

$$
\mathbf{V}=\frac{m_{1} \mathbf{v}_{1}+m_{2} \mathbf{v}_{2}}{\left(m_{1}+m_{2}\right)},
$$

es la velocidad del centro de masa del sistema en interacción.

Asumiendo que la colisión es elástica en el centro de masa $C_{M}$, i.e.

$$
u_{2}^{\prime 2}=u_{2}^{2},
$$

tal que la transferencia de energía en el sistema de laboratorio $C_{L}$ está dada por

$$
T=\frac{m_{2}}{2}\left(v_{2}^{\prime 2}-v_{2}^{2}\right)=m_{2} \mathbf{V} \cdot\left(\mathbf{u}_{2}^{\prime}-\mathbf{u}_{2}\right) .
$$

donde se ha hecho uso de la ecuación (2.3).

En el marco de referencia moviéndose con el proyectil $C_{P}$, una partícula del blanco tiene una velocidad inicial

$$
\mathbf{w}_{2}=\mathbf{v}_{2}-\mathbf{v}_{1} .
$$


Ahora, el flujo de partículas que inciden sobre el proyectil por unidad de tiempo y área con velocidad entre $\mathbf{w}_{2} \mathrm{y} \mathbf{w}_{2}+d \mathbf{w}_{2}$ en este sistema de referencia es

$$
n_{2}\left(\mathbf{v}_{2}-\mathbf{v}_{1}\right) f\left(\mathbf{v}_{2}\right) d^{3} v_{2}=n_{2} \mathbf{w}_{2} f\left(\mathbf{w}_{2}+\mathbf{v}_{1}\right) d^{3} w_{2}
$$

donde $n_{2}$ es el número de átomos del blanco por unidad de volumen.

Sea $d \sigma_{0}\left(w_{2}, \theta\right)$ la sección diferencial de transporte para un evento de dispersión entre $\theta$ y $\theta+d \theta$ en el sistema de referencia del centro de masa $C_{M}$, la cual nos representa la probabilidad de que la partícula sea dispersada. Con lo cual, el flujo total de partículas dispersadas en un tiempo $d t$ será

$$
n_{2} w_{2} d t f\left(\mathbf{w}_{2}+\mathbf{v}_{1}\right) d^{3} w_{2} d \sigma_{0}\left(w_{2}, \theta\right),
$$

que dividiendo entre el flujo total de partículas incidentes -en el centro de masa$n_{2} v_{1} d t$, uno encuentra la sección transversal aparente para un evento de dispersión $(\theta, d \theta)$ entre el proyectil y todos los electrones del blanco con velocidad dentro de $\left(\mathbf{w}_{2}, d^{3} w_{2}\right)$

$$
\frac{w_{2}}{v_{1}} f\left(\mathbf{w}_{2}+\mathbf{v}_{1}\right) d^{3} w_{2} d \sigma_{0}\left(w_{2}, \theta\right)
$$

que en términos de las velocidades del sistema de laboratorio $C_{L}$ es

$$
d \sigma=\frac{\left|\mathbf{v}_{1}-\mathbf{v}_{2}\right|}{v_{1}} f\left(\mathbf{v}_{2}\right) d^{3} v_{2} d \sigma_{0}\left(\left|\mathbf{v}_{1}-\mathbf{v}_{2}\right|, \theta\right),
$$

en donde hemos usado la relación entre las velocidades del sistema de laboratorio y el sistema que se mueve con el proyectil, ec. (2.7).

La pérdida de energía de un proyectil por trayectoria recorrida está dada por

$$
\frac{d E}{d x}=-n_{2} S_{e}
$$

donde

$$
S_{e}=\int T d \sigma
$$

es la sección de frenamiento electrónico. Así, combinando las ecuaciones (2.6), y (2.11) en la ecuación anterior obtenemos que

$$
S_{e}\left(v_{1}\right)=m_{2} \int d^{3} v_{2} f\left(\mathbf{v}_{2}\right) \frac{\left|\mathbf{v}_{1}-\mathbf{v}_{2}\right|}{v_{1}} \int d \sigma_{0}\left(\left|\mathbf{v}_{1}-\mathbf{v}_{2}\right|, \theta\right) \mathbf{V} \cdot\left(\mathbf{u}_{2}^{\prime}-\mathbf{u}_{2}\right) .
$$


Ahora, por definición, el ángulo de dispersión en el centro de masa está dado por

$$
\cos \theta=\frac{\left(\mathbf{u}_{2} \cdot \mathbf{u}_{2}^{\prime}\right)}{u_{2}^{2}}
$$

para colisiones elásticas. En el caso de simetría azimutal en la dispersión de $\mathbf{u}_{2}$, solo contribuyen las proyecciones de $\mathbf{u}_{2}^{\prime}$ sobre $\mathbf{u}_{2}$, i.e.

$$
\begin{aligned}
\left(\mathbf{u}_{2}^{\prime}-\mathbf{u}_{2}\right)_{\|} & =u_{2}^{\prime} \cos \theta \hat{\mathbf{u}}_{2}-\mathbf{u}_{2} \\
& =-\mathbf{u}_{2}(1-\cos \theta),
\end{aligned}
$$

con lo que la segunda integración en la ec. (2.14) toma la forma

$$
\begin{aligned}
\int d \sigma_{0}\left(\left|\mathbf{v}_{1}-\mathbf{v}_{2}\right|, \theta\right)\left(\mathbf{u}_{2}^{\prime}-\mathbf{u}_{2}\right) & =-\mathbf{u}_{2} \int d \sigma_{0}\left(\left|\mathbf{v}_{1}-\mathbf{v}_{2}\right|, \theta\right)(1-\cos \theta) \\
& =-\mathbf{u}_{2} \sigma^{(1)}\left(\left|\mathbf{v}_{1}-\mathbf{v}_{2}\right|\right)
\end{aligned}
$$

donde $\sigma^{(1)}(v)=\int d \sigma_{0}(v, \theta)(1-\cos \theta)$ es la sección transversal de transporte de primer orden [28]. Sustituyendo la ec. (2.17) en (2.14) obtenemos

$$
S_{e}\left(v_{1}\right)=\int d^{3} v_{2} f\left(\mathbf{v}_{2}\right) \mu \mathbf{V} \cdot\left(\mathbf{v}_{1}-\mathbf{v}_{2}\right) \frac{\left|\mathbf{v}_{1}-\mathbf{v}_{2}\right|}{v_{1}} \sigma^{(1)}\left(\left|\mathbf{v}_{1}-\mathbf{v}_{2}\right|\right),
$$

donde hemos hecho uso de la relación (2.3) y donde $\mu=m_{1} m_{2} /\left(m_{1}+m_{2}\right)$ es la masa reducida entre el proyectil y los electrones del blanco.

Consideremos el límite de altas velocidades, $v_{1} \gg v_{2}$. Esto nos define practicamente a los electrones del medio en reposo, i.e. nos define una sección de frenamiento $S_{0}\left(v_{1}\right)$ en reposo dada por

$$
\begin{aligned}
S_{0}\left(v_{1}\right) & =\int d^{3} v_{2} f\left(\mathbf{v}_{2}\right) \mu\left[\frac{m_{1} \mathbf{v}_{1}}{m_{1}+m_{2}}\right] \cdot \mathbf{v}_{1} \sigma^{(1)}\left(v_{1}\right) \\
& =\frac{m_{1}^{2} m_{2}}{\left(m_{1}+m_{2}\right)^{2}} v_{1}^{2} \sigma^{(1)}\left(v_{1}\right) \int d^{3} v_{2} f\left(\mathbf{v}_{2}\right)
\end{aligned}
$$

que haciendo uso de la normalización de la distribución de velocidades del blanco [ec. (2.2)] encontramos que

$$
S_{0}\left(v_{1}\right)=\frac{m_{1} m_{2}}{\left(m_{1}+m_{2}\right)^{2}} m_{1} v_{1}^{2} \sigma^{(1)}\left(v_{1}\right)
$$


la cual nos permite eliminar la sección de transporte transversal de primer orden con lo que obtenemos

$$
S\left(v_{1}\right)=\int d^{3} v_{2} f\left(\mathbf{v}_{2}\right)\left(\mathbf{v}_{1}+\frac{m_{2}}{m_{1}} \mathbf{v}_{2}\right) \cdot \frac{\left(\mathbf{v}_{1}-\mathbf{v}_{2}\right)}{v_{1}\left|\mathbf{v}_{1}-\mathbf{v}_{2}\right|} S_{0}\left(\left|\mathbf{v}_{1}-\mathbf{v}_{2}\right|\right)
$$

que nos relaciona la sección de frenamiento del sistema de laboratorio $S_{e}\left(v_{1}\right)$ con la sección de frenamiento en la que los electrones del blanco están en reposo. Notemos de ésto que las cantidades importantes en la descripción de la pérdida de energía son: la distribución de velocidades de los electrones que conforman el blanco $f\left(\mathbf{v}_{2}\right)$, la sección de frenamiento para blancos en reposo $S_{0}$ y si utilizamos el modelo de Bethe para velocidades del proyectil mayores que las velocidades orbitales de los electrones del blanco, entonces debemos considerar el análisis de la energía media de excitación $I_{2}$ del blanco (ver Apéndice A). En la siguiente sección se discute la manera de incorporar estas cantidades dentro de la Teoría cinética de una manera autoconsistente.

\subsection{Modelo OLPA/FSGO}

\subsubsection{Aproximación de Plasma Local (LPA)}

En 1947, Kramers [29] obtuvo la pérdida de energía de una partícula pesada cargada incidente en un gas de electrones libres en reposo como

$$
\frac{d E}{d x}=-\frac{4 \pi Z_{1}^{2} e^{4}}{m_{e} v_{1}^{2}} n_{2} \ln \frac{2 m_{e} v^{2}}{\hbar \omega_{p}} .
$$

En esta ecuación, $n_{2}$ es la densidad electrónica del gas, $\omega_{p}$ la frecuencia de plasma del gas de electrones dada por $\omega_{p}=\sqrt{4 \pi e^{2} n_{2} / m_{e}}$. Posteriormente Lindhard y Scharff [30] generalizaron los resultados de Kramers a un gas de electrones inhomogéneo haciendo una aproximación local, i.e. , el frenamiento en cualquier punto $\mathbf{r}$ en el gas inhomogéneo se supone que es el mismo que el frenamiento en un gas homogéneo con densidad $\rho(\mathbf{r})$ en ese punto. Así, promediando la expresión de Kramers sobre un solo átomo, Lindhard y Scharff obtuvieron

$$
L_{B}=\frac{1}{Z_{2}} \int d^{3} r \rho(\mathbf{r}) \ln \frac{2 m_{e} v_{1}^{2}}{\hbar \omega_{p}(\mathbf{r})},
$$


o una energía media de excitación dada por

$$
\ln I=\frac{1}{Z_{2}} \int d^{3} r \rho(\mathbf{r}) \ln \left(\chi \hbar \omega_{p}(\mathbf{r})\right) .
$$

Este es el modelo original de la teoría LPA. El parámetro ad hoc $\chi$ es indeterminado en esta aproximación pero fue introducido por Lindhard y Scharff para considerar las excitaciones colectivas - e.g. polarizabilidad. Ellos argumentan que el valor de $\chi$ debe ser aproximadamente $\sqrt{2}$. Obsérvese que las ecs. (2.22) y (2.23) son funciones de la densidad electrónica local.

\subsubsection{Aproximación de plasma local por orbital (OLPA)}

En 1982 John R. Sabin y Jens Oddershede (SO) [31, 32] generalizaron el modelo de la aproximación de plasma local aplicándolo a la contribución de los orbitales; Ellos notaron que si se utiliza una misma energía media de excitación común para todas las capas igual a la energía media de excitación del sistema, se obtenían correcciones no realistas en la región de bajas velocidades para el poder de frenamiento, por lo que introdujeron el formalismo del tipo de capas que describiremos a continuación. En una representación de partícula independiente, e.g. Hartree-Fock, se puede separar el logaritmo de la energía media de excitación total en las contribuciones por capas, por lo que SO definieron

$$
\ln I_{2}=\frac{1}{Z_{2}} \sum_{k} n_{k} \ln I_{k},
$$

donde la suma se extiende sobre todas las capas ocupadas, siendo los $n_{k}$ "factores de peso" para cada orbital $k$ y que no necesariamente son la población electrónica del orbital [33], los cuales para un sistema neutro satisfacen

$$
\sum_{k} n_{k}=Z_{2}
$$

lo cual a su vez, nos permite definir la sección transversal de frenamiento electrónico como

$$
S_{e}\left(v_{1}\right)=\sum_{k} S_{e, k}\left(v_{1}\right),
$$

donde $S_{e, k}$ es la contribución de cada orbital $k$. 
Posteriormente, ellos mismos (OS) [44] extienden su modelo incluiyendo en $S_{e}$ la contribución de los enlaces y carozos de un blanco molecular dado. En éste caso se requieren dos cantidades importantes, la energía media de excitación orbital para enlaces y carozos, y la distribución de velocidades orbitales para los electrones del blanco. De acuerdo a OS, la enegía media de excitación de los carozos $\left(I_{\text {carozo }}\right)$ se puede obtener a partir de su correspondiente valor del carozo atómico $1 s^{2}$. La enegía media de excitación para los enlaces $\left(I_{\text {enlaces }}\right)$ se lleva a cabo mediante el uso de una regla tipo Bragg para la energía media de excitación molecular $\left(I_{\text {molecular }}\right)$ tal que

$$
\ln I_{\text {molecula }}=\frac{1}{N_{2}}\left[\sum_{i}^{\text {carozos }} \omega_{i} \ln I_{i}^{\text {carozos }}+\sum_{j}^{\text {enlaces }} \omega_{j} \ln I_{j}^{\text {enlaces }}\right]
$$

donde $N_{2}$ es el número total de electrones en la molécula y $\omega_{k}$ la población electrónica de cada enlace y carozo. De aquí que la energía media de excitación de los enlaces se puede obtener directamente utilizando datos de $I_{\text {molecula }}$ teóricos o experimentales. Por otra parte, la distribución de velocidades de los electrones se puede obtener a partir de los perfiles Compton isotrópicos (ICP) derivados a partir de cálculos ab initio o mediante resultados experimentales y recurriendo a la relación entre la distribución de ímpetu isotrópico $\rho(q)$ y los ICP $J_{0}(q)$

$$
\rho(q)=-\frac{1}{2 q} \frac{d J_{0}}{d q}
$$

Usando valores precisos para $J_{0}(q)$ e $I_{\text {molecula }}$ en la implementación orbital de la teoría cinética, OS obtienen un excelente acuerdo con los valores experimentales disponibles de ICP e $I_{\text {molecula }}$ para protones en varios blancos moleculares.

Mas recientemente, un método alternativo de la implementación orbital de la teoría cinética ha sido propuesto por Meltzer, Sabin y Trickey (MST) [35] a través de una generalización en la descripción de la densidad en la aproximación de plasma local por orbital (Orbital Local Plasma Approximation -OLPA). Además, MST tratan el caso del frenamiento de protones por blancos atómicos usando densidades orbitales atómicas calculadas en la aproximación de densidad de spín local a la teoría de funcionales de la densidad. Su estimación para la sección transversal de frenamiento muestra un buen acuerdo con los cálculos llevados a cabo posteriormente por OS [32] basados en funciones de onda de Hartree-Fock-Slater [36] para el 
cálculo de la energía media de excitación y la distribución de velocidades orbitales.

Por lo tanto, para poder implementar la teoría cinética junto con la OLPA, debemos conocer los orbitales que conforman la estructura electrónica de nuestro sistema. En el caso que nos ocupa, recurrimos a los Orbitales Esféricos Gaussianos Flotantes (FSGO) que es una representación localizada de los orbitales moleculares, además de permitirnos un tratamiento autoconsistente de todas las cantidades que aparecen en el cálculo. Como se verá más adelante, ésto nos permite explorar las capacidades de la implementación OLPA en la teoría cinética para el tratamiento del poder de frenamiento molecular, sin necesidad de recurrir a información externa alguna, tal como lo son resultados experimentales.

\subsection{Orbitales moleculares FSGO}

Con el fín de que nuestra representación converja a la representación enlace-valencia, utilicemos un modelo para orbitales moleculares propuesto por A. A. Frost [37]. Consideremos un sistema electrónico con número par de electrones, $2 n$, distribuidos en $n$ orbitales localizados representados por la función $\Psi_{i}$, con $i=1 \ldots n$, los cuales no necesariamente deben ser ortogonales. En esta representación, cada orbital describe a electrones de capa interna (carozos), electrones en los orbitales de enlace y electrones en los orbitales de pares solitarios.

Cada orbital localizado está representado por una función esférica Gaussiana flotante

$$
\Psi_{i}\left(\mathbf{r}-\mathbf{R}_{i}\right)=\left(\frac{2}{\pi \sigma_{i}^{2}}\right)^{3 / 4} e^{-\left|\mathbf{r}-\mathbf{R}_{i}\right|^{2} / \sigma_{i}^{2}}
$$

donde $\sigma_{i}$ es el parámetro relacionado con el "radio orbital" y $\mathbf{R}_{i}$ la variable que describe la posición del centro de la gaussiana. Con ésto, la función de onda electrónica total es un solo determinante de Slater para capas cerradas. Cada orbital individual es no ortogonal, por lo tanto existe una matriz de traslape $S$ definida por

$$
S_{i j}=\int \Psi_{i}^{*} \Psi_{j} d^{3} r .
$$

La matriz de traslape inversa, $T=S^{-1}$ es aquella cuyos elementos matriciales $T_{i j}$ aparecen en la fórmula de energía

$$
E=2 \sum_{j, k}\langle j|h| k\rangle T_{j, k}+\sum_{k, l, p, q}\langle k p \mid l q\rangle\left[2 T_{k l} T_{p q}-T_{k q} T_{l p}\right]
$$


donde el primer término corresponde a la contribución de la energía cinética del sistema electrónico ( $h$ siendo el operador de energía cinética de un electrón) y el segundo término es la contribución de la interacción Coulombica entre los electrones. Minimizando mediante un método variacional con respecto a todos los parámetros, el radio orbital y la posición de las coordenadas nucleares, se predice la estructura electrónica y geométrica del sistema.

Una de las ventajas del modelo de Frost, es que permite "visualizar" los orbitales de la molécula mediante esferas, esto se debe a que el radio orbital $\sigma_{i}$ representa el radio de una esfera que contiene cerca del $74 \%$ de la densidad de carga electrónica y además, dar un tratamiento analítico más extenso en nuestro problema.

\subsection{Implementación al poder de frenamiento}

Para evaluar la sección de frenamiento electrónico dentro de la teoría cinética desde un punto de vista matemático más simplificado, analicemos el resultado de Sigmund [ec. (2.20)] desde el sistema de referencia que se mueve con el proyectil. Haciendo la suposición de que el proyectil es mucho más pesado que los electrones del blanco $m_{1} \gg m_{2}$, entonces la ec. (2.20) se reduce a $[17]$

$$
S_{e}\left(v_{1}\right)=-\int f\left(\mathbf{v}_{1}-\mathbf{v}^{\prime}\right) \frac{\mathbf{v}_{1} \cdot \mathbf{v}^{\prime}}{v_{1} v^{\prime}} S_{0}\left(v^{\prime}\right) d^{3} v^{\prime}
$$

Lo cual nos dice que $S_{e}$ es el promedio sobre $S_{0}$ pesado por la distribución de velocidades electronicas del medio, proyectada a lo largo de la dirección de movimiento del proyectil.

Recurriendo a la descomposición orbital descrita anteriormente y suponiendo una distribución orbital isotrópica de velocidades para los dispersores en el sistema de referencia del laboratorio, la ecuación anterior se puede escribir como

$$
S_{e}\left(v_{1}\right)=\sum_{k} S_{e, k}\left(v_{1}\right)
$$

donde $S_{e, k}$ es la contribución de cada orbital, la cual está dada por

$$
S_{e, k}\left(v_{1}\right)=\frac{4 \pi e^{4} Z_{1}^{2} Z_{2}}{m_{e} v_{1}^{2}} L_{k}\left(v_{1}\right)
$$


donde $m_{e}$ es la masa del electrón, $e$ es su carga eléctrica y $L_{k}$ es el número de frenamiento el cual está determinado por la aproximación en el cálculo de la sección de frenamiento electrónico en reposo.

Prosiguiendo con la implementación de OS, el número de frenamiento para los dispersores del blanco en reposo $L_{k}^{0}$ se toma de la fórmula de Bethe en la forma

$$
L_{k}^{0}(v)=\frac{n_{k}}{N_{2}} \ln \left[\frac{2 m_{e} v^{2}}{I_{2, k}}\right] \Theta\left(v-\alpha_{k}\right)
$$

donde $\alpha_{k}=\left(I_{2, k} / 2 m_{e}\right)^{(1 / 2)}$ e $I_{2, k}$ es la energía media de excitación del orbital $k$. La función de Heaviside $\Theta\left(v-\alpha_{k}\right)$ nos asegura el uso de la fórmula de Bethe en su rango de aplicabilidad. Con ésto, el número de frenamiento dentro de la teoría cinética en términos de la población electrónica $n_{k}$ del orbital $k$-ésimo está dado por

$$
L_{e, k}\left(v_{1}\right)=\frac{\pi v_{1}^{2} n_{k}}{N_{2}} \int_{\alpha_{k}}^{\infty} \ln \left[\frac{2 m_{e} v^{\prime 2}}{I_{2, k}}\right] d v^{\prime} \int_{0}^{\pi} f_{k}\left(\sqrt{v_{1}^{2}+v^{\prime 2}-2 v_{1} v^{\prime} \cos \theta}\right) d\left(\sin ^{2} \theta\right) .
$$

Notemos que la distribución de velocidades isotrópica en el sistema del laboratorio es solo isotrópica azimutalmente en el sistema de referencia que se mueve con el proyectil, como era de esperarse.

Ahora, necesitamos calcular la distribución de velocidades de los electrones del blanco. Empleando FSGO, tomemos la transformada de Fourier de la ecuación (2.29), de lo cual obtenemos

$$
\Phi_{k}(\mathbf{v})=\left(\frac{\pi \sigma_{k}^{2} m_{e}}{2 \hbar^{2}}\right)^{3 / 4} \pi^{-3 / 2} e^{-i \frac{m_{e} \mathbf{v} \cdot \mathbf{R}_{k}}{\hbar}} e^{-\left(\frac{m_{e} \sigma_{i} v}{2 \hbar}\right)^{2}},
$$

con lo que la distribución de velocidades del orbital $k$-ésimo es:

$$
\begin{aligned}
f_{k}(v) & =\Phi_{k}^{*}(v) \Phi_{k}(v) \\
& =\left(\frac{\pi \sigma_{k}^{2} m_{e}}{2 \hbar^{2}}\right)^{3 / 2} \pi^{-3} e^{-\frac{m_{e}^{2} \sigma_{k}^{2} v^{2}}{2 \hbar^{2}}} .
\end{aligned}
$$

Así, en el sistema de referencia del proyectil tenemos que

$$
f_{k}\left(\left|\mathbf{v}_{1}-\mathbf{v}^{\prime}\right|\right)=\frac{\beta_{k}^{3}}{(2 \pi)^{-3 / 2}} e^{-\frac{\beta_{k}^{2}}{2}\left(v_{1}^{2}+v^{\prime 2}-2 v_{1} v^{\prime} \cos \theta\right)}
$$


donde $\beta_{k}=m_{e} \sigma_{k} / \hbar \mathrm{y} \mathbf{v}^{\prime}$ es la velocidad relativa entre los electrones del blanco y el proyectil. Usando esta ecuación en la ecuación (2.36) y después de algunas manipulaciones algebraicas encontramos que la contribución de cada orbital a $S_{e}$ es

$$
S_{e, k}(u)=2^{5 / 2} \sqrt{\pi} N_{1}^{2} n_{k} \sigma_{k}^{3} e^{-\frac{\sigma_{k}^{2} u^{2}}{2}} G_{k}\left(\sigma_{k}, I_{2, k}, u\right),
$$

con

$$
G_{k}\left(\sigma_{k}, I_{2, k}, u\right)=\int_{\alpha_{k}}^{\infty} e^{-\frac{\sigma_{k}^{2} x^{2}}{2}} \ln \left[\frac{4 R_{h} x^{2}}{I_{2, k}}\right]\left[\frac{\cosh \left(\sigma_{k}^{2} u x\right)}{\sigma_{k}^{2} u x}-\frac{\operatorname{senh}\left(\sigma_{k}^{2} u x\right.}{\left(\sigma_{k}^{2} u x\right)^{2}}\right] d x
$$

donde $R_{h}$ es la energía de Rydberg, $u=v_{1} / v_{0}, x=v^{\prime} / v_{0}$ y $v_{0}$ es la velocidad de Bohr.

Lo que nos resta calcular es la energía media de excitación $I_{2, k}$ por orbital. De nueva cuenta recurrimos al modelo orbital FSGO, en el cual la densidad electrónica del orbital $k$ esta dada por

$$
\rho_{k}(\mathbf{r})=n_{k}\left(\frac{2}{\pi \sigma_{k}^{2}}\right)^{3 / 2} e^{\left[-2 \frac{\left(\mathbf{r}-\mathbf{R}_{k}\right)^{2}}{\sigma_{k}^{2}}\right]}
$$

que sustituyendo en la ecuación (2.23) obtenemos la frecuencia de plasma local asociada al orbital y por lo tanto la energía media de excitación de éste orbital en la aproximación de plasma local por orbital

$$
I_{2, k}=\left(\frac{2048}{\pi}\right)^{1 / 4} \sqrt{n_{k}} \frac{e^{-3 / 4}}{\sigma_{k}^{3 / 2}} R_{h} .
$$

La expresión anterior es muy simple y como vemos, todo depende de parámetros que se obtienen a partir de primeros principios, como son el radio del orbital $\sigma_{k} \mathrm{y}$ la población electrónica de dicho orbital $n_{k}$.

\subsection{Análisis de los resultados}

Empezaremos por analizar las predicciones de la energía media de excitación dada por la ecuación (2.44) para el caso atómico y compararemos los resultados con los obtenidos por MST [35].

En la tabla 2.1 se muestran los valores obtenidos para las energía media de excitación a partir de la ecuación (2.44) y usando los radios orbitales disponibles 


\begin{tabular}{lcccrrr}
\hline Atomo & Orbital & $\sigma_{k}\left(a_{0}\right)$ & $\omega_{k}$ & $I_{k}^{L P A / F S G O}$ & $I_{k}^{M S T a}$ & $I_{k}^{O S b}$ \\
\hline$H e^{c}$ & $1 s$ & 1.142 & 2 & 37.63 & 33.66 & 38.83 \\
$C^{d}$ & $1 s$ & 0.328 & 2 & 244.49 & 220.78 & 451.34 \\
$N^{c}$ & $1 s$ & 0.277 & 2 & 315.03 & 283.07 & 590.00 \\
$O^{e}$ & $1 s$ & 0.240 & 2 & 390.62 & 349.72 & 729.41 \\
$N a^{f}$ & $1 s$ & 0.171 & 2 & 649.49 & 577.82 & 1110.36 \\
& $2 s$ & 0.799 & 2 & 64.31 & 47.12 & 119.24 \\
& $2 p$ & 0.799 & 6 & 111.38 & 93.51 & 124.41 \\
$M g^{f}$ & $1 s$ & 0.156 & 2 & 745.39 & 661.87 & 1243.15 \\
& $2 s$ & 0.697 & 2 & 78.93 & 56.77 & 151.05 \\
& $2 p$ & 0.697 & 6 & 136.70 & 118.75 & 169.86 \\
$A l^{f}$ & $1 s$ & 0.143 & 2 & 849.31 & 749.74 & 1371.04 \\
& $2 s$ & 0.618 & 2 & 94.53 & 66.87 & 187.14 \\
& $2 p$ & 0.618 & 6 & 163.74 & 145.18 & 221.15 \\
$S i^{f}$ & $1 s$ & 0.132 & 2 & 957.65 & 841.22 & 1497.54 \\
& $2 s$ & 0.553 & 2 & 111.68 & 77.39 & 226.08 \\
& $2 p$ & 0.553 & 6 & 193.44 & 172.81 & 278.63 \\
$P^{f}$ & $1 s$ & 0.123 & 2 & 1064.66 & 936.24 & 1618.33 \\
& $2 s$ & 0.502 & 2 & 129.13 & 88.56 & 266.84 \\
& $2 p$ & 0.502 & 6 & 223.65 & 201.65 & 342.50 \\
$S^{f}$ & $1 s$ & 0.115 & 2 & 1177.67 & 1034.70 & 1733.33 \\
& $2 s$ & 0.458 & 2 & 148.17 & 100.29 & 308.23 \\
& $2 p$ & 0.458 & 6 & 256.64 & 231.89 & 412.71 \\
$C l^{f}$ & $1 s$ & 0.107 & 2 & 1312.18 & 1136.50 & 1844.43 \\
& $2 s$ & 0.421 & 2 & 168.13 & 112.49 & 349.09 \\
& $2 p$ & 0.421 & 6 & 291.21 & 263.31 & 489.36 \\
\hline \multirow{4}{*}{} & & & & & &
\end{tabular}

Tabla 2.1: Energía media de excitación $I_{k}^{L P A / F S G O}$ calculada en éste trabajo para las capas $K$ y $L$ para varios sistemas atómicos para los cuales se dispone de los parámetros FSGO. Además, se muestran los cálculos correspondientes al modelo OLPA $\left(I_{k}^{M S T}\right)$ y los cálculos HF de OS $\left(I_{k}^{O S}\right)$ (ver texto). Las energias estan dadas en $\mathrm{eV}$.

[38] y la descomposición orbital de OS [ver ec. (2.24)] para varios sistemas atómicos. Además en la misma tabla mostramos los resultados obtenidos por MST y OS $\left(I_{2, k}^{M S T}\right.$ y $\left.I_{2, k}^{O S}\right)$ [referencias [35] y [32]] y en la que se muestra un razonable acuerdo tanto cualitativo como cuantitativo entre los resultados obtenidos en éste trabajo y los métodos más elaborados de MST y OS. Adicionalmente se observa que las energías medias de excitación obtenidas con los orbitales FSGO son mayores que las 


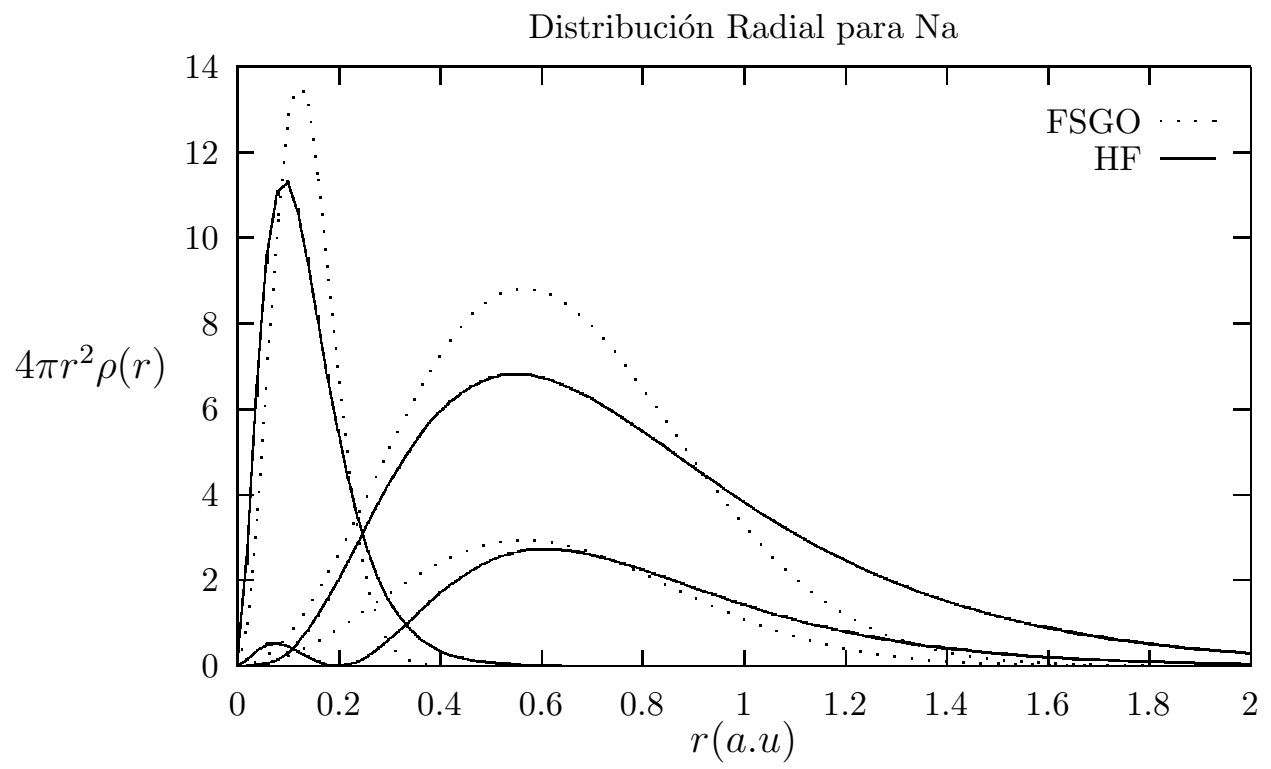

Figura 2.1: Distribución de carga radial para los orbitales $1 s, 2 s$ y $2 p$ en el $\mathrm{Na}$ calculados a partir de un solo orbital FSGO (linea punteada) y comparado con los orbitales Hartree-Fock-Slater (curva continua).

obtenidas por MST y OS, i.e. $I_{2, k}^{O L P A / F S G O}>I_{2, k}^{M S T}$. Una posible explicación a éste comportamiento la podemos obtener si recordamos que la distribución electrónica de los orbitales FSGO está más localizada que la distribución electrónica en una representación más realista (el radio orbital representa el 74\% de la distribución electrónica en un orbital FSGO).

Para ejemplificar esta aserción, en la figura (2.1) hemos graficado el comportamiento de la distribución radial de carga para los orbitales 1s, 2s, y 2p para el Sodio (Na) usando ambas representaciones, FSGO y HFS (linea punteada y continua, respectivamente) en la que notamos la naturaleza compacta de la distribución electrónica FSGO en contraste con HFS, con lo cual, de acuerdo a la ecuación (2.44), entre más compacto sea el orbital, más grande será su contribución a la energía media de excitación.

Aunque no contamos con parámetros para la capa $M$, podemos hacer una estimación de la la energía media de excitación de el orbital $3 s$ en $N a$ y $M g$ mediante la energía media de excitación total $I[I(N a)=99.2 \mathrm{eV} ; I(M g)=101.1 \mathrm{eV}]$ calculada de la densidad de carga atómica total [35]. Usando la descomposición orbital propuesta por MST y nuestos valores de $I_{k}$ tomados de la tabla 2.1 para las 


\begin{tabular}{|c|c|c|c|c|c|c|c|}
\hline Molécula & enlace & $\sigma_{k}\left(a_{0}\right)$ & $\omega_{k}$ & $I_{k}^{L P A / F S G O}$ & $I_{k}^{O S a}$ & $I^{L P A / F S G O}$ & $I_{\text {molecula }}^{\text {exp }}$ \\
\hline $\mathrm{CH}_{4}^{b}$ & $C-H$ & 1.694 & 2 & 20.83 & 21.20 & 34.09 & $41.70^{g}$ \\
\hline \multirow{2}{*}{$\mathrm{C}_{2} \mathrm{H}_{4}$} & $C-H$ & 1.642 & 2 & 21.83 & & \multirow{2}{*}{42.12} & \multirow{2}{*}{$49.93^{g}$} \\
\hline & $C=C$ & 1.794 & 4 & 27.03 & 27.93 & & \\
\hline \multirow{2}{*}{$\mathrm{C}_{2} \mathrm{H}_{2}^{c}$} & $C-H$ & 1.581 & 2 & 23.10 & & \multirow{2}{*}{53.13} & \\
\hline & $C \equiv C$ & 1.781 & 6 & 33.47 & & & \\
\hline \multirow{2}{*}{$\mathrm{C}_{2} \mathrm{H}_{6}^{c}$} & $C-H$ & 1.695 & 2 & 20.81 & & \multirow{2}{*}{36.16} & \multirow{2}{*}{$45.40^{g}$} \\
\hline & $C-C$ & 1.646 & 2 & 21.75 & 33.63 & & \\
\hline \multirow{2}{*}{$\begin{array}{l}C_{2} H_{6}^{c} \\
\text { Ciclo-propano }\end{array}$} & $C-H$ & 1.683 & 2 & 21.04 & & \multirow{2}{*}{38.12} & \multirow{2}{*}{ - } \\
\hline & $C-C$ & 1.770 & 2 & 19.50 & & & \\
\hline \multirow{5}{*}{$\begin{array}{l}\mathrm{C}_{2} \mathrm{H}_{6}^{d} \\
\text { Propileno }\end{array}$} & $C 1-H$ & 1.649 & 2 & 21.69 & & \multirow{5}{*}{40.96} & \multirow{5}{*}{$50.05^{b}$} \\
\hline & $C 2-H$ & 1.645 & 2 & 21.77 & & & \\
\hline & $C 3-H$ & 1.688 & 2 & 20.94 & & & \\
\hline & $C 1=C 2$ & 1.796 & 4 & 26.99 & & & \\
\hline & $C 2-C 3$ & 1.620 & 2 & 22.27 & & & \\
\hline \multirow{2}{*}{$\mathrm{H}_{2} \mathrm{O}^{b}$} & $O-H$ & 1.308 & $\overline{2}$ & 30.70 & 40.68 & \multirow{2}{*}{50.53} & \multirow{2}{*}{$72.46^{2}$} \\
\hline & $O_{L P}$ & 1.331 & 2 & 29.91 & & & \\
\hline \multirow{2}{*}{$\mathrm{NH}_{3}^{b}$} & $N-H$ & 1.554 & 2 & 23.71 & 29.49 & \multirow{2}{*}{39.23} & \multirow{2}{*}{$53.69^{2}$} \\
\hline & $N_{L P}$ & 1.627 & 2 & 22.13 & & & \\
\hline \multirow{2}{*}{$\mathrm{O}_{2}^{e}$} & $O=O$ & 1.264 & 4 & 45.71 & & \multirow{2}{*}{65.44} & \multirow{2}{*}{$95.02^{i}$} \\
\hline & $O_{L P}$ & 1.271 & 2 & 32.05 & & & \\
\hline \multirow{2}{*}{$N_{2}^{e}$} & $N \equiv N$ & 1.449 & 6 & 45.61 & & \multirow{2}{*}{66.93} & \multirow{2}{*}{$81.84^{i}$} \\
\hline & $N_{L P}$ & 1.489 & 2 & 25.28 & & & \\
\hline$H_{2}^{f}$ & $H-H$ & 1.772 & 2 & 19.47 & & 19.47 & $19.26^{j}$ \\
\hline
\end{tabular}

${ }^{a}$ Ref. [44]; ${ }^{b}$ Ref. [39]; ${ }^{c}$ Ref. [40]; ${ }^{d}$ Ref. [41]; ${ }^{e}$ Ref. [42]; ${ }^{f}$ Ref. [43]; ${ }^{g}$ Ref. [45]; ${ }^{h}$ Ref. [46]; ${ }^{i}$ Ref. [47]; ${ }^{j}$ Ref. [48].

Tabla 2.2: Energía media de excitación orbital $\left(I_{k}^{L P A / F S G O}\right)$ y total $\left(I^{L P A / F S G O}\right)$ calculadas en éste trabajo para diferentes enlaces y pares solitarios. Además se muestran los resultados de OS $\left(I^{O S}\right)$ y los valores experimentales aceptados para $I_{\text {molecula }}$. Todas las energias estan en $\mathrm{eV}$.

capas $K$ y $L$, obtenemos $I_{3 s}(N a)=2.75 \mathrm{eV}$ y $I_{3 s}(M g)=7.1 \mathrm{eV}$, en buén acuerdo con $I_{3 s}^{M S T}(\mathrm{Na})=2.98 \mathrm{eV}$ y $I_{3 s}^{M S T}(\mathrm{Mg})=6.08 \mathrm{eV}$ obtenidos por los autores antes citados. De aquí, es claro que la Ec. (2.44) nos permite describir razonablemente $I_{k}$ dentro del espíritu de la OLPA.

Por otro lado, en la tabla 2.2 se muestran los valores para las energías medias de excitación orbital $\left(I_{k}^{L P A / F S G O}\right)$ calculada por medio de la ec. (2.44) para diferentes enlaces y pares solitarios que aparecen en varias moléculas, para las cuales se dispone 
de los parámetros FSGO [39]-[43]. Los valores correspondientes a los carozos se muestran en la tabla 2.1. Además, por comparación se muestran los resultados reportados por OS [32] y por completez, también se muestran los radios FSGO, población orbital $n_{k}$ y la energía media de excitación total $I^{L P A / F S G O}$ obtenidas en este trabajo comparándose con los resultados de otras fuentes [45]-[48].

Nótese el efecto del entorno químico en la energía media de excitación para el mismo tipo de enlaces en las moléculas de diferente estructura. Por ejemplo, en una estructura anular tal como la del ciclopropano $\left(C_{3} H_{6}\right)$ y su isómero alqueno, el propileno, el valor de $I_{2, k}$ para el enlace $C-C$ difieren en un factor de 1.14. Como otro ejemplo, consideremos el caso del acetileno, en el cual el enlace $C-H$ tiene el más grande valor para $I_{2, k}$ entre las moléculas de hidrocarburos mostradas en esta tabla y lo mismo podemos decir del caso de la molécula de agua $\mathrm{H}_{2} \mathrm{O}$ y la del oxígeno $\mathrm{O}_{2}$ en la cual los orbitales de pares solitarios (LP) difieren ligeramente debido al entorno químico, inherente al grado de compactez del orbital como se puede ver de la ecuación (2.44) y donde claramente se ve que entre mayor sea el radio orbital menor será el valor de $I_{2, k}$. En conexión con esto vemos de la misma tabla que $I_{C-H}<I_{C-C}<I_{C=C}<I_{C \equiv C}$ en contraste con los resultados de OS [44] quienes dan $I_{C-H}<I_{C=C}<I_{C-C}$. Aunque se puede observar un buen acuerdo entre los resultados mostrados aquí y lo obtenidos por OS para las energías de excitación de $C-H$ y $C=C$, para el caso de $C-C$ los valores FSGO son marcadamente diferentes. Analizando aun con más detalle vemos que por ejemplo, en el caso de las energías de excitación para los enlaces $N-H$ y $O-H$ las diferencias que se observan en este trabajo con las reportadas por OS se pueden explicar en nuestro tratamiento mediante la existencia de los orbitales de pares solitarios como entidades moleculares distintas en contraste con OS en el cual ellos incluyen los electrones de pares solitarios en los enlaces de $O-H$. Otra característica a notar es que en promedio los valores obtenidos en éste trabajo están en un 20 a $30 \%$ por abajo de los valores reportados en la literatura como aceptados.

Una vez que podemos obtener las energías medias de excitación, las podemos usar en las ecuaciones para $S_{e}$ y obtener la sección de frenamiento electrónico del enlace molecular [ver ec. (2.41)]. Así, después de una integración numérica obtenemos las contribuciones a los carozos, enlaces moleculares y pares solitarios a $S_{e}$ como función de la velocidad del proyectil incidente en un blanco, los cuales mostramos 


\begin{tabular}{|rrrrrrrrr|}
\hline & $C-H$ & $C-C$ & $C=C$ & $C \equiv C$ & $O-H$ & $O-O$ & $N-H$ & $N \equiv N$ \\
u[u.a.] & Molecula fuente & & & & & & \\
\cline { 2 - 9 } & $\mathrm{CH}_{4}$ & $\mathrm{C}_{2} \mathrm{H}_{6}$ & $\mathrm{C}_{2} \mathrm{H}_{4}$ & $\mathrm{C}_{2} \mathrm{H}_{2}$ & $\mathrm{H}_{2} \mathrm{O}$ & $\mathrm{O}_{2}$ & $\mathrm{NH}_{3}$ & $N_{2}$ \\
\hline 0.5 & 5.916 & 5.527 & 8.998 & 10.001 & 3.189 & 3.992 & 4.822 & 6.265 \\
& $(5.007)$ & $(3.197)$ & $(7.453)$ & & $(4.015)$ & & $(4.403)$ & \\
\hline 1.0 & 9.889 & 9.314 & 16.175 & 19.173 & 5.658 & 7.472 & 8.246 & 12.105 \\
& $(8.363)$ & $(5.251)$ & $(12.522)$ & & $(7.272)$ & & $(7.662)$ & \\
\hline 1.5 & 10.835 & 10.349 & 18.763 & 23.727 & 6.905 & 9.778 & 9.441 & 16.044 \\
& $(9.406)$ & $(6.666)$ & $(14.888)$ & & $(9.320)$ & & $(9.286)$ & \\
\hline 2.0 & 9.666 & 9.366 & 17.285 & 22.911 & 6.952 & 10.553 & 8.760 & 17.099 \\
& $(8.952)$ & $(6.502)$ & $(14.500)$ & & $(9.989)$ & & $(9.233)$ & \\
\hline 2.5 & 7.946 & 7.772 & 14.454 & 19.697 & 6.276 & 10.087 & 7.414 & 15.962 \\
& $(7.181)$ & $(5.848)$ & $(12.650)$ & & $(9.510)$ & & $(8.238)$ & \\
\hline 3.0 & 6.440 & 6.329 & 11.838 & 16.383 & 5.385 & 9.010 & 6.104 & 13.966 \\
& $(5.871)$ & $(4.976)$ & $(10.612)$ & & $(8.535)$ & & $(7.046)$ & \\
\hline 4.0 & 4.384 & 4.327 & 8.163 & 11.474 & 3.856 & 6.737 & 4.212 & 10.238 \\
& $(4.035)$ & $(3.548)$ & $(7.528)$ & & $(6.556)$ & & $(5.075)$ & \\
\hline 8.0 & 1.526 & 1.513 & 2.898 & 4.155 & 1.407 & 2.574 & 1.487 & 3.870 \\
& $(1.578)$ & $(1.451)$ & $(2.979)$ & & $(2.686)$ & & $(1.893)$ & \\
\hline 10.0 & 1.064 & 1.055 & 2.027 & 2.918 & 0.988 & 1.823 & 1.038 & 2.738 \\
& $(1.129)$ & $(1.026)$ & $(2.142)$ & & $(1.910)$ & & $(1.331)$ & \\
\hline
\end{tabular}

Tabla 2.3: Contribución a la sección de frenamiento electrónico de los carozos, enlaces y pares solitarios en función de la velocidad para protones incidentes en algunas moléculas selectas en la fase gaseosa. Las unidades están en $10^{-15} \mathrm{eV} \mathrm{cm}$ 2 /enlace. Los valores entre paréntesis son de la ref. [44]

en las Tablas 2.3 y 2.4. Los valores en paréntesis corresponden a los cálculos de OS [44] para la misma situación y la tendencia del comportamiento de los resultados la podemos entender en términos de la energía media de excitación como ya lo discutimos anteriormente.

Así, tomando los valores obtenidos con la ec. (2.41) y usando la ec. (2.33) obtenemos la contribución a la sección de frenamiento electrónico para algunas moléculas, en particular, en las figuras (2.2) y (2.3) mostramos los resultados obtenidos para protones incidentes sobre varios blancos gaseosos comparados con algunos datos experimentales [49]-[53] y con las estimaciones teóricas de OS [16]. Así, como ejemplos, para los casos de $\mathrm{CH}_{4}, \mathrm{~N}_{2}$ y $\mathrm{H}_{2}$ las gráficas se contruyeron a partir de estos valores, sin embargo para tomar en cuenta el entorno químico para el mismo tipo 


\begin{tabular}{|rrrrrrrrr|}
\hline \multirow{2}{*}{$\begin{array}{c}\text { L u.a. }] \\
\end{array}$} & $\begin{array}{c}\text { LP1 } \\
\text { Molecula fuente }\end{array}$ & & LP3 & LP4 & $\mathrm{C}$ & $\mathrm{O}$ & $\mathrm{N}$ & $H-H$ \\
\cline { 2 - 9 } $\mathrm{H}_{2} \mathrm{O}$ & $\mathrm{O}_{2}$ & $\mathrm{NH}_{3}$ & $\mathrm{~N}_{2}$ & & & & $H_{2}$ \\
\hline 0.5 & 3.326 & 2.975 & 5.377 & 4.354 & 0.096 & 0.042 & 0.061 & 6.578 \\
& & & & & $(0.053)$ & $(0.024)$ & $(0.035)$ & \\
\hline 1.0 & 5.882 & 5.306 & 9.089 & 7.523 & 0.0189 & 0.083 & 0.122 & 10.850 \\
& & & & & $(0.106)$ & $(0.049)$ & $(0.069)$ & \\
\hline 1.5 & 7.136 & 6.536 & 10.157 & 8.745 & 0.278 & 0.123 & 0.179 & 11.613 \\
& & & & & $(0.160)$ & $(0.073)$ & $(0.104)$ & \\
\hline 2.0 & 7.131 & 6.659 & 9.244 & 8.307 & 0.360 & 0.162 & 0.234 & 10.132 \\
& & & & & $(0.213)$ & $(0.097)$ & $(0.138)$ & \\
\hline 2.5 & 6.395 & 6.070 & 7.701 & 7.140 & 0.434 & 0.198 & 0.285 & 8.214 \\
& & & & & $(0.264)$ & $(0.121)$ & $(0.172)$ & \\
\hline 3.0 & 5.461 & 5.258 & 6.284 & 5.932 & 0.498 & 0.232 & 0.331 & 6.610 \\
& & & & & $(0.314)$ & $(0.144)$ & $(0.205)$ & \\
\hline 3.5 & 4.603 & 4.466 & 5.164 & 4.922 & 0.553 & 0.263 & 0.372 & 5.391 \\
& & & & & $(0.358)$ & $(0.167)$ & $(0.236)$ & \\
\hline 4.0 & 3.894 & 3.795 & 4.304 & 4.156 & 0.596 & 0.290 & 0.407 & 4.472 \\
& & & & & $(0.396)$ & $(0.188)$ & $(0.264)$ & \\
\hline 8.0 & 1.415 & 1.393 & 1.508 & 1.467 & 0.616 & 0.386 & 0.487 & 1.547 \\
& & & & & $(0.454)$ & $(0.274)$ & $(0.350)$ & \\
\hline 10.0 & 0.993 & 0.979 & 1.052 & 1.026 & 0.522 & 0.368 & 0.441 & 1.076 \\
& & & & & $(0.396)$ & $(0.265)$ & $(0.323)$ & \\
\hline
\end{tabular}

Tabla 2.4: Contribución a la sección de frenamiento electrónico de los carozos, enlaces y pares solitarios en función de la velocidad para protones incidentes en algunas moléculas selectas en la fase gaseosa. Las unidades están en $10^{-15} \mathrm{eV} \mathrm{cm}$ 2 /enlace. Los valores entre paréntesis son de la ref. [44]

de enlace en diferente estructura molecular, hemos usado los valores dados en las tablas 2.3 y 2.4 para las cantidades que entran en el cálculo de $S_{e}$ para los casos de $\mathrm{C}_{2} \mathrm{H}_{2}$ y $\mathrm{C}_{2} \mathrm{H}_{4}$. Notemos que dentro de la simplicidad de éste modelo, los resultados obtenidos están en buen acuerdo con los resultados experimentales. Sin embargo, debemos notar que en nuestro modelo no hemos considerado correcciones de Barkas y Bloch $[54,55]$ y por lo tanto debemos tener cuidado al comparar con el experimento principalmente en la región alrededor del máximo y bajas velocidades. Esto podría ser una explicación del porqué en el caso de metano $\mathrm{CH}_{4}$ y el acetileno $\mathrm{C}_{2} \mathrm{H}_{4}$ nuestros resultados difieren ligeramente en el máximo de la curva. En la mismas gráficas, mostramos los resultados obtenidos por OS dentro de las mismas 


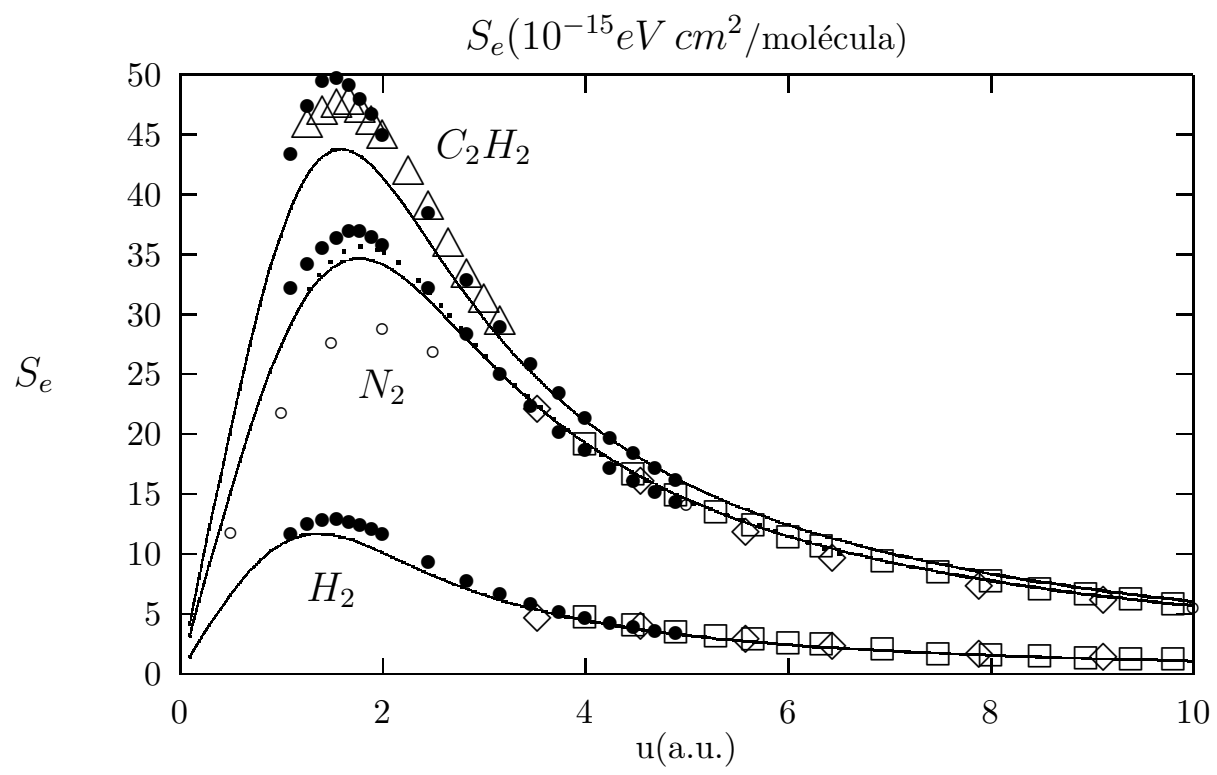

Figura 2.2: Sección de frenamiento electrónico para protones incidentes en $\mathrm{H}_{2}, \mathrm{~N}_{2} \mathrm{y}$ $\mathrm{C}_{2} \mathrm{H}_{2}$. Línea continua: predicciones de éste trabajo; Circulos abiertos: Predicciones teorícas de la ref. [34]. Línea semi-continua: Mejor ajuste al experimento para $\mathrm{N}_{2}$ de la ref. [49]. Datos experimentales: • [50], $\triangle[51], \square[52], \diamond[53]$

condiciones de dispersión en la aproximación Bethe/Born. Como se puede observar de las figuras 2.2 y 2.3 nuestros valores de la sección de frenamiento electrónico estan sistemáticamente sobre aquellos de OS por un $15 \%$ en la región de velocidades alrededor del máximo, uniendose las curvas a altas velocidades. Este efecto ya había sido observado por MST en su análisis de la sección de frenamiento electrónico para el caso atómico. Una posible explicación de éste comportamiento se debe al uso del factor $\chi$ en la ecuación (2.23) el cual hemos considerado el mismo para todos los orbitales $\left(\chi_{k}=1\right)$.

\subsection{Conclusiones}

Los resultados mostrados en este capítulo indican lo adecuado del uso de la representación FSGO de los orbitales moleculares para el estudio de la sección de frenamiento electrónico para protones dentro del modelo de la teoría cinética y la implementación de la aproximación orbital de plasma local (OLPA) debida a Meltzer, Sabin y Trickey. Aunque el acuerdo que se observa entre las predicciones 


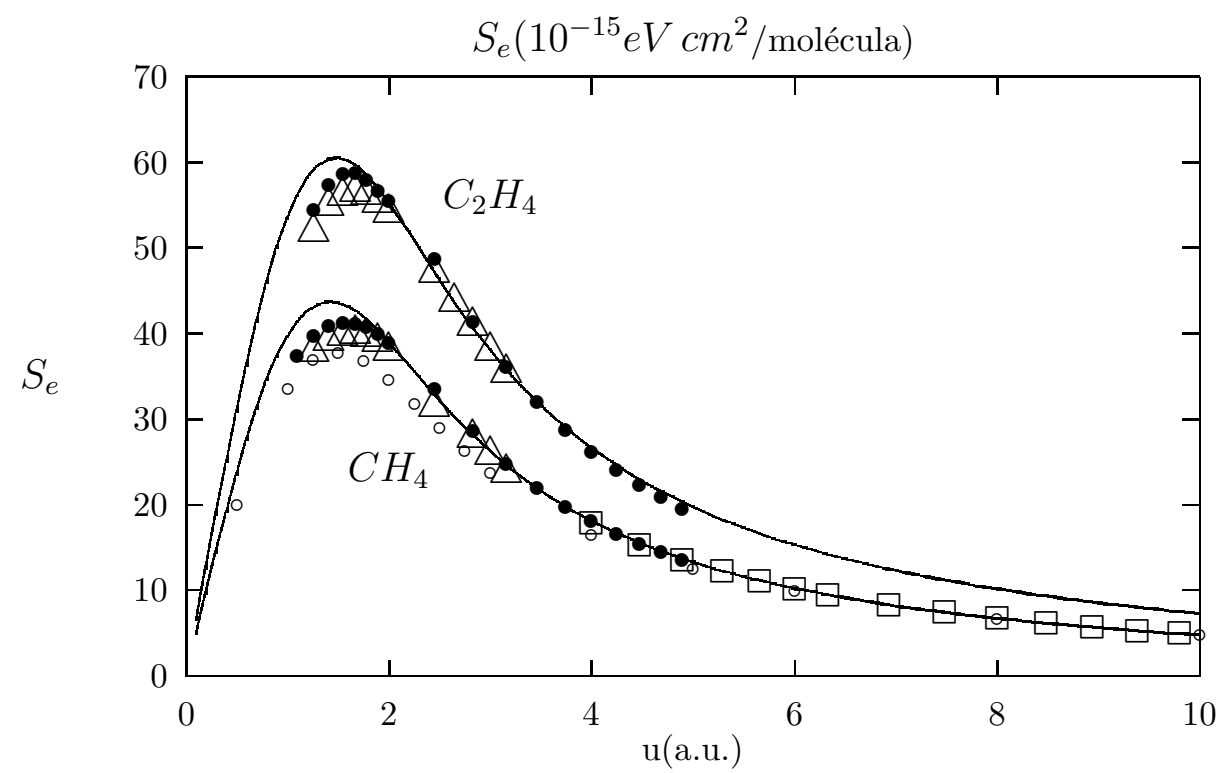

Figura 2.3: Sección de frenamiento electrónico para protones incidentes en $\mathrm{CH}_{4} \mathrm{y}$ $\mathrm{C}_{2} \mathrm{H}_{4}$. Los símbolos tienen en mismo significado que en la figura 2.2

de este modelo y el experimento para la sección transversal de frenamiento molecular para protones incidentes en blancos gaseosos es bueno, es necesario tomar en cuenta los términos de corrección de Barkas y Bloch tal que se pueda concluir de manera definitiva. Por otro lado, aún prevalece el problema de mejorar las predicciones del modelo OLPA para las energías de excitación orbital y total. Esto nos conduce a un análisis más detallado de la densidad electrónica de los electrones del blanco mediante el uso de bases FSGO ortogonales, así como la implementación de un modelo alternativo de la aproximación de plasma local por orbital.

Todo este tratamiento se ha llevado a cabo bajo la suposición de que los proyectiles no tienen estructura electrónica (protones desnudos). Para obtener información de la contribución de la estructura electrónica del proyectil, es necesario hacer un tratamiento de primeros principios. Esta es la contribución del siguiente capítulo. 


\section{Capítulo 3}

\section{Extensión de la Teoría de Bethe a proyectiles con estructura}

\subsection{Resumen}

En éste capítulo se extiende la teoría de Bethe para la pérdida de energía de particulas cargadas rápidas colisionando con blancos atómicos para el caso en que el proyectil tiene electrones ligados, de tal manera que se incorporan las excitaciones e ionizaciones tanto del proyectil como las del blanco.

\subsection{Introducción}

Cuando un proyectil penetra en un medio material ocurren un sinúmero de procesos tanto en el blanco como en el proyectil. Uno de ellos, la captura y pérdida de electrones por el proyectil con lo cual, al menos temporalmente, el ion puede tener algunos electrones ligados aún si $v>v_{e}[56,57]$. Estos procesos tienen una alta probabilidad, en particular para el rango de velocidades $v_{0}<v<v_{0} Z_{1}^{2 / 3}$ [6], donde $v_{0}$ es la velocidad de Bohr. Así para obtener una descripción más apropiada de la sección transversal de frenamiento uno debe de considerar la estructura electrónica del proyectil y del blanco. El primer estudio en esta dirección fue llevado a cabo a mediados de los 50's por Bates y Griffing [58], quienes calcularon la sección de frenamiento para el más simple de los procesos de colisión: dos átomos de Hidrógeno. Este trabajo formó la base para estudios sistemáticos de interacciones más generales del tipo átomo-átomo [59]. 
Durante los últimos años, la dependencia del estado de carga en el frenamiento electrónico ha sido un área de investigación muy activa. Tratamientos previos basados en reglas de escalamiento promedio tales como la teoría de carga efectiva [60], aunque útiles para estimar el poder de frenamiento promedio de iones pesados, aún representa una descripción incompleta de los procesos de captura y pérdida de electrones en la colisión. Recientemente modelos más realistas y sofisticados basados en soluciones numéricas a la ecuación de Schrödinger [61, 62], principios variacionales dependientes del tiempo [63], o teorías de funcionales de la densidad [64] han sido sugeridos. Sin embargo estos modelos son sólo factibles actualmente para proyectiles de tipo Hidrogenoide o tipo Helio (partículas alfa), por lo que existe la necesidad de una teoría más general para tratar proyectiles en forma.

Dentro del espíritu de la teoría de Bethe han habido varios esfuerzos para tomar en cuenta el frenamiento de iones con estructura electrónica parcial. Kim y Cheng [56] sugirieron un esquema donde el poder de frenamiento se obtiene a partir de la fórmula original de Bethe, reemplazando el número atómico del proyectil $Z_{1}$, y la energía media de excitación $I_{2}$ del blanco por un número atómico efectivo $Z_{\mathrm{ef}}$ y una energía media de excitación efectiva $I_{\text {ef }}$, respectivamente. Ambos $Z_{\text {ef }}$ e $I_{\text {ef }}$ son derivables a partir de las propiedades del proyectil y del blanco que se pueden obtener de un tratamiento de primeros principios.

Similarmente, Arnau y Echenique [65] propusieron una manera de introducir la estructura electrónica del proyectil dentro de la teoría dieléctrica de frenamiento. Más recientemente, Moneta y Czerbniak [66] han usado la primera aproximación de Born [67] para estudiar el poder de frenamiento de iones parcialmente desnudos basandose en una descripción semiclásica del parámetro de impacto. En su modelo, los electrones del proyectil pueden ser excitados o ionizados pero sin considerar intercambio electrónico.

En este capítulo, propondremos una modificación a la teoría de Bethe dentro de la primera aproximación de Born para describir la contribución al poder de frenamiento de iones con estructura electrónica parcial dependiente de la velocidad del proyectil. En contraste con el tratamiento de Moneta y Czerbniak [66], nuestra formulación no depende del parámetro de impacto y el número de electrones se obtiene a partir del criterio adiabático de Bohr [68]. Debido al carácter Coulombiano de la interacción y al hecho de que no se está considerando intercambio de carga, se 
puede separar de manera natural la contribución al frenamiento en contribuciones del proyectil y del blanco.

\subsection{El poder de frenamiento: Desarrollo General}

Consideremos un proyectil parcialmente desnudo moviéndose con una velocidad $v$ (en el sistema de laboratorio), masa $M_{1}$, carga nuclear $Z_{1} e$, y con $N_{1}$ electrones ligados descritos por el eigenestado $\left|n_{0}\right\rangle$; el cual colisiona con un blanco estacionario de masa $M_{2}$ que tiene $N_{2}$ electrones ligados en un estado inicial denotado por $\left|m_{0}\right\rangle$. El proyectil es dispersado dentro de un elemento de ángulo sólido $d \Omega$ a lo largo de una dirección con ángulos polares $(\theta, \varphi)$ medido en el sistema de laboratorio. Supongamos que el sistema proyectil-blanco sufre una transición a los estados finales $|n\rangle$ y $|m\rangle$, respectivamente, con energias $E_{n}$ y $E_{m}$. Entonces la energía cinética del proyectil se reduce en una cantidad $\left(E_{n}-E_{n_{0}}\right)+\left(E_{m}-E_{m_{0}}\right)=\hbar\left(w_{n n_{0}}+w_{m m_{0}}\right) \mathrm{si}$ no consideramos intercambio electrónico.

El Hamiltoniano que describe el sistema es

$$
\hat{H}=\hat{H}_{0}+\hat{H}_{\text {int }}
$$

donde $\hat{H}_{\text {int }}$ es el Hamiltoniano de interacción entre el proyectil y el blanco y $\hat{H}_{0}$ es el Hamiltoniano del sistema no perturbado dado por

$$
\hat{H}_{0}=\hat{H}_{p}+\hat{H}_{b} \quad,
$$

con $\hat{H}_{b}$ el Hamiltoniano que describe al blanco y $\hat{H}_{p}$ el Hamiltoniano que describe al proyectil como una partícula libre que se mueve con velocidad $v$, incluyendo su estructura electrónica, i.e.

$$
\hat{H}_{p}=\hat{H}_{p, l i b r e}+\hat{H}_{p, e s t r u c t} \quad .
$$

El Hamiltoniano de interacción entre el proyectil y el blanco esta dado por

$$
\begin{aligned}
\hat{H}_{i n t}(\mathbf{x}, \mathbf{y}, \mathbf{R})= & \frac{Z_{1} Z_{2} e^{2}}{R}-\sum_{i=1}^{N_{2}} \frac{Z_{1} e^{2}}{\left|\mathbf{R}-\mathbf{y}_{i}\right|} \\
& -\sum_{i=1}^{N_{1}} \frac{Z_{2} e^{2}}{\left|\mathbf{R}+\mathbf{x}_{i}\right|}+\sum_{i, j=1}^{N_{1} N_{2}} \frac{e^{2}}{\left|\mathbf{y}_{j}-\mathbf{R}-\mathbf{x}_{i}\right|} .
\end{aligned}
$$


donde el primer término corresponde a la interacción entre el núcleo del proyectil y el núcleo del blanco separados una distancia $|\mathbf{R}|$, el segundo es la interacción entre el núcleo del proyectil y los electrones del blanco (descritos por el vector de posición $\mathbf{y}_{i}$ respecto al centro de masa del blanco), el tercer término es la interacción de los electrones del proyectil (denotados por el vector de posición $\mathbf{x}_{i}$ respecto al centro de masa del proyectil) y el núcleo del blanco y, finalmente, el cuarto término es la interacción entre los electrones del proyectil y los electrones del blanco.

Si suponemos que $\hat{H}_{\text {int }}$ es suficientemente pequeño para ser considerado como una perturbación, lo cual nos limita a proyectiles y blancos no tan pesados (cargas nucleares no muy grandes o distancias $\mathbf{R}$ muy grandes -transferencias de ímpetu pequeñas) podemos utilizar teoría de perturbaciones dependiente del tiempo. Al tiempo $t_{0}$ el sistema se encuentra en un eigenestado del Hamiltoniano sin perturbar, $\hat{H}_{0}$, i.e. la función de onda del sistema $\Psi\left(t_{0}\right)$ es de la forma

$$
\Psi\left(t_{0}\right)=u_{\mu_{0}} \quad,
$$

donde

$$
\hat{H}_{0} u_{\mu_{0}}=E_{\mu_{0}} u_{\mu_{0}}
$$

y $\mu_{0}$ representa el estado en el que se encuentra todo el sistema.

Por la forma del Hamiltoniano $\hat{H}_{0}$ y el hecho de que no estamos considerando intercambio electrónico entre el proyectil y el blanco, vemos que la función de onda total no-perturbada es un producto del tipo Hartree, i.e.

$$
\left|\mu_{0}\right\rangle=\left|k_{0} n_{0} m_{0}\right\rangle
$$

donde

$$
\left|k_{0}\right\rangle=\frac{1}{(2 \pi)^{(3 / 2)}} e^{i \mathbf{k}_{0} \cdot \mathbf{R}}
$$

es la función de onda para el proyectil incidiendo como partícula libre, $\left|n_{0}\right\rangle$ la función de onda asociada a la estructura electrónica del proyectil y $\left|m_{0}\right\rangle$ la función de onda que describe la estructura electrónica del blanco. Consecuentemente la energía del sistema esta dada por

$$
E_{\mu_{0}}=E_{k_{0}}+E_{n_{0}}+E_{m_{0}} \quad,
$$

donde $E_{k_{0}}$ es la energía cinética del movimiento del proyectil, $E_{n_{0}}$ y $E_{m_{0}}$ la de la estructura del proyectil y blanco respectivamente. 
De acuerdo a la teoría de perturbaciones dependiente del tiempo, las eigenfunciones del problema que satisfacen la ecuación de Schrödinger dependiente del tiempo

$$
\left(\hat{H}_{0}+\hat{H}_{i n t}\right) \Psi=i \hbar \frac{\partial \Psi}{\partial t}
$$

se puede construir expandiendo $\Psi$ en términos de eigenfunciones del sistema no perturbado, i.e.

$$
\Psi(\mathbf{x}, \mathbf{y}, \mathbf{R}, t)=\sum_{\nu} a_{\nu}(t) e^{-i E_{\nu}\left(t-t_{0}\right) / \hbar} u_{\nu}
$$

lo que sustituyendo en la ec. (3.10) nos conduce a una ecuación diferencial para los coeficientes

$$
i \hbar \frac{d a_{\mu}}{d t}=\sum_{\nu} a_{\nu}\left\langle\nu\left|\hat{H}_{i n t}\right| \mu\right\rangle e^{-i\left(E_{\nu}-E_{\mu}\right)\left(t-t_{0}\right) / \hbar} \quad .
$$

Ahora, introduciendo la suposición de pequeña perturbación i.e. consideramos al sistema casi sin disturbarse durante la colisión

$$
a_{\mu}(t)=1 \quad\left(\mu=\mu_{0}\right)
$$

$\mathrm{y}$

$$
a_{\mu}(t)<<1 \quad\left(\mu \neq \mu_{0}\right) .
$$

Con esta suposición, la probabilidad de encontrar al sistema en el estado $\mu$ al tiempo $t$ esta dada por

$$
\left|a_{\mu}(t)\right|^{2}=\frac{4}{E_{\mu \mu_{0}}^{2}} \mid\left\langle\mu\left|\hat{H}_{i n t}\right| \mu_{0}\right\rangle^{2} \operatorname{sen}^{2}\left[\frac{E_{\mu \mu_{0}}\left(t-t_{0}\right)}{2 \hbar}\right],
$$

donde

$$
E_{\mu \mu_{0}}=E_{\mu}-E_{\mu_{0}}
$$

Por lo tanto la probabilidad de transición por unidad de tiempo para tiempos muy grandes $\left(t-t_{0}\right) \rightarrow \infty$ esta dada por

$$
W_{\mu \mu_{0}}=\lim _{t \rightarrow \infty} \frac{d}{d t}\left|a_{\mu \mu_{0}}(t)\right|^{2}=\frac{2 \pi}{\hbar}\left|\left\langle\mu\left|\hat{H}_{i n t}\right| \mu_{0}\right\rangle\right|^{2} \delta\left(E_{\mu}-E_{\mu_{0}}\right)
$$

que es la Regla de Oro de Fermi.

La función Delta garantiza la conservación de la energía total del sistema puesto que

$$
E_{\mu}=E_{k}+E_{n}+E_{m}=E_{k_{0}}+E_{n_{0}}+E_{m_{0}}=E_{\mu_{0}}
$$


Si el proyectil no es capturado por el blanco, su energía cinética como partícula libre estará en el contínuo y emergerá después dentro de un ángulo sólido $d \Omega$ alrededor de $\mathbf{k}$, por lo que

$$
E_{k}=\frac{\hbar^{2} \mathbf{k}^{2}}{2 M_{1}}
$$

Entonces

$$
k^{2} d k d \Omega=d^{3} k=d \Omega \frac{k M_{1}}{\hbar^{2}} d E_{k} \quad,
$$

con lo que el cambio diferencial en la probabilidad de transición por unidad de tiempo para una dispersión dentro del ángulo sólido $d \Omega$ es

$$
d W_{\mu \mu_{0}}=\frac{2 \pi M_{1}}{\hbar^{3}} \int\left|\left\langle\mu\left|\hat{H}_{i n t}\right| \mu_{0}\right\rangle\right|^{2} \delta\left(E_{k}+E_{n}+E_{m}-E_{k_{0}}-E_{n_{0}}-E_{m_{0}}\right) k d \Omega d E_{k}
$$

que integrando sobre las energias nos conduce a

$$
d W_{\mu \mu_{0}}=\frac{2 \pi M_{1}}{\hbar^{3}}\left|\left\langle\mu\left|\hat{H}_{\text {int }}\right| \mu_{0}\right\rangle\right|^{2} k d \Omega
$$

Ahora, el flujo de partícula incidentes esta dado por

$$
\mathbf{J}_{i n c}=\frac{\hbar \mathbf{k}_{0}}{M_{1}(2 \pi)^{3}}
$$

tal que la sección transversal diferencial de dispersión se obtiene como

$$
\frac{d \sigma_{\mu \mu_{0}}}{d \Omega}=\frac{d W_{\mu \mu_{0}} / d \Omega}{\left|\mathbf{J}_{\text {inc }}\right|}=\frac{(2 \pi)^{4} M_{1}^{2}}{\hbar^{4}} \frac{k}{k_{0}}\left|\left\langle\mu\left|\hat{H}_{\text {int }}\right| \mu_{0}\right\rangle\right|^{2}
$$

Con lo que [ver Apéndice A, ec. (A.5)] la sección de frenamiento inelástico es

$$
S_{e}(v)=\frac{(2 \pi)^{4} M_{1}^{2}}{\hbar^{4}} \sum_{n, m} \int\left[\left(E_{n}-E_{n_{0}}\right)+\left(E_{m}-E_{m_{0}}\right)\right]\left|\left\langle n m k\left|\hat{H}_{i n t}\right| n_{0} m_{0} k_{0}\right\rangle\right|^{2} \frac{k}{k_{0}} d \Omega,
$$

donde se ha expandido el estado $|\mu\rangle=|k n m\rangle$ y donde la suma es sobre los estados excitados del proyectil y blanco $n, m$.

Para calcular $S_{e}(v)$ explícitamente primero analicemos el elemento de matríz

$$
\begin{aligned}
\left\langle n m k\left|\hat{H}_{i n t}\right| n_{0} m_{0} k_{0}\right\rangle=\frac{1}{(2 \pi)^{3}} & \int e^{(-i \mathbf{q} \cdot \mathbf{R})} \psi_{m}^{*}\left(\mathbf{y}_{1}, \ldots, \mathbf{y}_{N_{2}}\right) \phi_{n}^{*}\left(\mathbf{x}_{1}, \ldots, \mathbf{x}_{N_{1}}\right) \\
& \times \hat{H}_{i n t}(\mathbf{x}, \mathbf{y}, \mathbf{R}) \psi_{m_{0}}\left(\mathbf{y}_{1}, \ldots, \mathbf{y}_{N_{2}}\right) \phi_{n_{0}}\left(\mathbf{x}_{1}, \ldots, \mathbf{x}_{N_{1}}\right) \\
& \times d \mathbf{x}_{1} \ldots d \mathbf{x}_{N_{1}} d \mathbf{y}_{1} \ldots d \mathbf{y}_{N_{2}} d \mathbf{R}
\end{aligned}
$$


donde $\hbar \mathbf{q}=\hbar\left(\mathbf{k}-\mathbf{k}_{0}\right)$ es el ímpetu transferido durante la colisión, con $\hbar \mathbf{k}_{0}$ el ímpetu inicial de proyectil y $\hbar \mathbf{k}$ el ímpetu final. Las funciones de onda $\psi$ y $\phi$ son las eigenfunciones electrónicas $|m\rangle$ y $|n\rangle$ en las coordenadas $\mathbf{y}_{i}$ y $\mathbf{x}_{i}$ para el proyectil y blanco respectivamente.

Siguiendo el procedimiento de Bethe (ver ref. [28]), podemos simplicar esta expresión llevando a cabo la integración en $\mathbf{R}$. Para ésto, vemos que el primer término de $\hat{H}_{\text {int }}$ en la ecuación (3.4) sólo depende de la distancia internuclear $R$ y debido a la ortogonalidad del estado base y el estado excitado tanto del proyectil como del blanco ese término no contribuye, con lo que sólo permanecen los términos que involucran la estructura electrónica del proyectil y blanco.

Utilizando la siguiente relación

$$
e^{-i \mathbf{q} \cdot \mathbf{R}}=-\frac{1}{q^{2}} \nabla^{2} e^{-i \mathbf{q} \cdot \mathbf{R}}
$$

y el hecho de que

$$
\nabla^{2} \frac{1}{\left|\mathbf{R}-\mathbf{r}_{i}\right|}=-4 \pi \delta\left(\mathbf{R}-\mathbf{r}_{i}\right)
$$

encontramos que integrando dos veces por partes la ec. (3.20) sobre $\mathbf{R}$ (vease por ejemplo la referencia [28]) obtenemos

$$
\begin{aligned}
& \left\langle n m k\left|\hat{H}_{i n t}\right| n_{0} m_{0} k_{0}\right\rangle=\frac{2 e^{2}}{(2 \pi)^{2} q^{2}}\left[Z_{12} T_{m m_{0}}(\mathbf{q}) \delta_{n n_{0}}+Z_{2}{ }_{1} T_{n n_{0}}(\mathbf{q}) \delta_{m m_{0}}-\right. \\
& \left.{ }_{1} T_{n n_{0}}(\mathbf{q}){ }_{2} T_{m m_{0}}(\mathbf{q})\right]
\end{aligned}
$$

con

$$
{ }_{1} T_{n n_{0}}(\mathbf{q})=\left\langle n\left|\sum_{j}^{N_{1}} e^{i \mathbf{q} \cdot \mathbf{x}_{j}}\right| n_{0}\right\rangle \text { y }{ }_{2} T_{m m_{0}}(\mathbf{q})=\left\langle m\left|\sum_{j}^{N_{2}} e^{i \mathbf{q} \cdot \mathbf{y}_{j}}\right| m_{0}\right\rangle
$$

los factores de forma atómicos $[69,70]$ de la transición entre el estado inicial y el estado final de el proyectil y blanco respectivamente. Introduciendo esta última ecuación en la sección de frenamiento electrónico [ec. (3.19)] y usando el hecho de que el elemento diferencial de ángulo sólido se puede escribir como $d \Omega=q d q d \phi / k k_{0}=d \mathbf{q} / k k_{0}$ tal que después de una breve simplificación algebráica [veáse el Apéndice B] 


$$
\begin{aligned}
S_{e}(v)= & \frac{2 e^{4}}{m_{e} v^{2}} \sum_{n, m}\left\{\int_{q_{\min }}^{q_{\max }}\left|Z_{2} \delta_{m_{0} m}-{ }_{2} T_{m_{0} m}(\mathbf{q})\right|^{2}{ }_{1} F_{n_{0} n}(\mathbf{q}) \frac{d \mathbf{q}}{q^{2}}\right. \\
& \left.+\int_{q_{\min }}^{q_{\max }}\left|Z_{1} \delta_{n_{0} n}-{ }_{1} T_{n_{0} n}(\mathbf{q})\right|^{2}{ }_{2} F_{m_{0} m}(\mathbf{q}) \frac{d \mathbf{q}}{q^{2}}\right\} \\
= & S_{e, 1}(v)+S_{e, 2}(v),
\end{aligned}
$$

y donde se ha hecho uso de la definición de las Tensiones de Oscilador Generalizado (GOS) [7] (veáse Apéndice C)

$$
\begin{aligned}
{ }_{1} F_{n_{0} n}(\mathbf{q}) & =\frac{2 m_{e}}{\hbar^{2} q^{2}}\left|{ }_{1} T_{n_{0} n}(\mathbf{q})\right|^{2} E_{n n_{0}}, \\
{ }_{2} F_{m_{0} m}(\mathbf{q}) & =\left.\left.\frac{2 m_{e}}{\hbar^{2} q^{2}}\right|_{2} T_{m_{0} m}(\mathbf{q})\right|^{2} E_{m m_{0}} .
\end{aligned}
$$

Al igual que en la teoría de Bethe, los límites de la integración en q de la ecuación (3.23) se obtienen de la cinemática del proceso de colisión. El mínimo ímpetu transferido se obtiene suponiendo que existe al menos un estado excitado en el sistema proyectil-blanco. El máximo ímpetu transferido a un electrón se obtiene como consecuencia de la conservación de energía y momento.

Así, para iones pesados se obtiene que [72]

$$
q_{\min }=\frac{E_{n n_{0}}+E_{m m_{0}}}{\hbar v}, \quad q_{\max }=\frac{2 m_{e} v}{\hbar}
$$

La ecuación (3.23) es aplicable al poder de frenamiento electrónico de iones con estructura electrónica dentro de la primera aproximación de Born. El primer término de esta ecuación contiene la cantidad ${ }_{1} F_{n_{0} n}$, que nos da la contribución al poder de frenamiento de las transiciones electrónicas del proyectil modulada por la estructura electrónica del blanco a través de los factores de forma correspondientes. De la misma manera, el segundo término contiene ${ }_{2} F_{m_{0} m}$ el cual describe la contribución de las transiciones electrónicas en el blanco modulada por la estructura electrónica del proyectil.

Conociendo los GOS para el proyectil y blanco, se puede obtener de manera inmediata $S_{e}(v)$ de la ecuación (3.23). Sin embargo, es necesario conocer el espectro 
completo de excitación para el proyectil y el blanco, lo cual no es tarea fácil y por lo tanto es necesario recurrir a métodos aproximados.

\subsubsection{La aproximación de Bethe}

Para poder derivar una expresión analítica de la sección de frenamiento electrónico $S_{e}(v)$ en la que se incluya la energía media de excitación del proyectil y blanco, es conveniente introducir la regla de suma de Bethe [7] para el proyectil y blanco (veáse Apéndice C)

$$
\sum_{n}{ }_{1} F_{n_{0} n}(\mathbf{q})=N_{1}, \quad \sum_{m}{ }_{2} F_{m_{0} m}(\mathbf{q})=N_{2} .
$$

la cual nos dice que la energía transferida al sistema sumada sobre todos los modos internos de excitación debe ser la misma que la energía transferida a $N$ electrones libres [73].

Suponiendo funciones de onda de partícula independiente tipo Hartree-Fock, se puede demostrar (veáse Apéndice D) que

$$
\begin{gathered}
\sum_{n}\left|Z_{1} \delta_{n_{0} n}-{ }_{1} T_{n_{0} n}(\mathbf{q})\right|^{2}=\left|Z_{1}-{ }_{1} T_{n_{0} n_{0}}(\mathbf{q})\right|^{2} \\
\sum_{m}\left|Z_{2} \delta_{m_{0} m}-{ }_{2} T_{m_{0} m}(\mathbf{q})\right|^{2}=\left|Z_{2}-{ }_{2} T_{m_{0} m_{0}}(\mathbf{q})\right|^{2},
\end{gathered}
$$

donde ${ }_{1} T_{n_{0} n_{0}}(\mathbf{q})$ y ${ }_{2} T_{m_{0} m_{0}}(\mathbf{q})$ son los factores de forma de el estado base para el proyectil y blanco respectivamente.

Siguiendo el desarrollo propuesto por Bethe [72] (ver Apéndice A), intercambiamos la suma sobre $(m, n)$ y la integración en la ecuación (3.23), y reemplazamos $q_{\text {min }}$ por un valor promedio apropiado a determinar, el cual es independiente de los estados de excitación de el sistema.

$$
q_{\min _{\mathrm{AV}}}=\frac{\left(E_{n n_{0}}+E_{m m_{0}}\right)_{\mathrm{AV}}}{\hbar v}=\frac{\epsilon}{\hbar v} .
$$

Usando así esta aproximación y las reglas de suma [ecs. (3.26) y (3.27)], obtenemos

$$
S_{e}(v)=\frac{2 e^{4}}{m_{e} v^{2}} \int_{\epsilon / \hbar v}^{2 m_{e} v / \hbar}\left\{N_{2}\left[Z_{1}-{ }_{1} T_{n_{0} n_{0}}(\mathbf{q})\right]^{2}+\right.
$$


34 CAPÍTULO 3. TEORÍA DE BETHE: PROYECTILES CON ESTRUCTURA

$$
\left.N_{1}\left[Z_{2}-{ }_{2} T_{m_{0} m_{0}}(\mathbf{q})\right]^{2}\right\} \frac{d \mathbf{q}}{q^{2}}
$$

Con lo cual hemos especificado el poder de frenamiento en términos de un parámetro aún indeterminado $\epsilon$, el cual depende de las propiedades del sistema en cuestion.

Podemos obtener una expresión para $\epsilon$ usando el hecho de que éste está definido tal que se permita el intercambio entre la sumatoria y la integración en la ec. (3.23). Siguiendo el procedimiento bosquejado en el apendice E, obtenemos que

$$
\epsilon=I_{2}^{1 / 1+\alpha} I_{1}^{\alpha / 1+\alpha}
$$

donde $I_{1}$ e $I_{2}$ son las energías medias de excitación de el proyectil y blanco respectivamente y $\alpha$ esta determinado por el número de electrones en el proyectil y blanco mediante

$$
\alpha=\frac{\left(Z_{2}-N_{2}\right)^{2} N_{1}}{\left(Z_{1}-N_{1}\right)^{2} N_{2}}
$$

Así, el poder de frenamiento en la aproximación de Bethe cuando se toman en cuenta las excitaciones e ionizaciones del proyectil y blanco esta dado por la ecuación (3.29), es decir, asumiendo velocidades $v$ no-relativistas más grandes que la velocidad orbital de los electrones ligados al proyectil y/o blanco.

Para no perder continuidad con el tratamiento se dejará para la sección 3.6 la discusión formal.

\subsection{Criterio adiabático para $N_{1}(v)$}

Hasta ahora hemos propuesto una modificación a la teoría de Bethe para el poder de frenamiento de proyectiles con estructura electrónica en su forma general ec. (3.23) y en la aproximación de Bethe ec. (3.29). Para poder hacer uso de los resultados es necesario conocer el número de electrones ligados al proyectil $N_{1}$, como función de la velocidad de éste.

Hasta ahora, hemos supuesto tácitamente que $N_{1}$ es una cantidad constante para todas las velocidades $v$ del proyectil. Sin embargo, ésta es una cantidad fluctuante, y la distribución de los electrones en un cierto tiempo está determinada por los procesos de pérdida y captura de electrones que tienen lugar a lo largo de la trayectoria del proyectil cuando éste penetra el medio material. Usualmente se establece un equilibrio dinámico entre estos procesos de pérdida y captura, permitiendo definir 
el número de electrones ligados al proyectil como una función de la velocidad del proyectil, $N_{1}=N_{1}(v)$.

En general, el cálculo de una distribución en equilibrio de electrones en el proyectil requiere una estimación detallada de la sección transversal de pérdida y captura de electrones. Sin embargo, el número de electrones en el proyectil se puede estimar haciendo uso de la suposición de que los electrones del proyectil son arrancados, si su velocidad orbital $v_{e}$ es menor que la velocidad del proyectil, mientras que los electrones con velocidad mayor que la velocidad orbital, $v_{e}>v$ son retenidos en la colisión, puesto que por ejemplo, viendo al proceso desde un sistema que se mueve con el proyectil, las colisiones son adiabáticas. Este es el criterio adiabático de Bohr [68]. Este criterio se puede re-escribir en la forma de una condición de potencial-energía [76],

$$
\frac{1}{2} m_{e} v^{2}+U\left(r_{c}\right)=0,
$$

donde $r_{c}$ es la distancia de un electrón ligado al núcleo del proyectil para el cual su velocidad es igual a la velocidad del proyectil $\left(v_{e}=v\right)$. Un electrón para el cual $r>r_{c}$ se ésta moviendo con $v_{e}<v$ y por lo tanto es arrancado del proyectil.

Con lo que el número de electrones arrancados del proyectil que se mueve con velocidad $v$ es

$$
Z_{1}-N_{1}(v)=\int_{r_{c}(v)}^{\infty} \rho_{e, 1}(r) d^{3} r,
$$

donde $r_{c}$ es solución a la ecuación (3.32) y $\rho_{e, 1}(r)$ es la densidad electrónica del proyectil neutro $\left(N_{1}=Z_{1}\right)$.

Para determinar $r_{c}$, y así el número de electrones $N_{1}(v)$, es necesario conocer $v_{e}$ como función de $r$. Para ésto, haremos uso de el modelo de Thomas-Fermi (TF) para el átomo [77], el cual describe a un sistema atómico con $N_{1}$ electrones ligados por medio de la densidad electrónica

$$
\rho_{e, 1}(r)=\frac{N_{1}}{4 \pi \Lambda_{1}^{2} r} \frac{d^{2} \Phi(x)}{d x^{2}},
$$

donde $x=r / \Lambda_{1}, \Phi(x)$ es la función de apantallamiento la cual es solución a la ecuación de TF, y donde $\Lambda_{1}$ es la longitud de apantallamiento la cual se considera como un parámetro variacional [vease ec. (3.36)] lo cual se obtiene minimizando la energía total 
36 CAPÍTULO 3. TEORÍA DE BETHE: PROYECTILES CON ESTRUCTURA

$$
E=E_{n e}+\lambda E_{e e}+E_{c i n}
$$

como una función del número de electrones $N_{1}$, con la energía potencial para la interacción núcleo-electrón

$$
E_{n e}=-Z_{1} e \int d^{3} r \frac{\rho_{e, 1}(r)}{r},
$$

la energía para la interacción electrón-electrón

$$
E_{e e}=\frac{1}{2} \int d^{3} r \int d^{3} r^{\prime} \frac{\rho_{e, 1}(r) \rho_{e, 1}\left(r^{\prime}\right)}{\left|\mathbf{r}-\mathbf{r}^{\prime}\right|}
$$

pesada por el parámetro $\lambda$ en una manera promedio para tomar en cuenta efectos de correlación e intercambio electrónico [60] y finalmente la energía cinética del gas de electrones

$$
E_{\text {cin }}=\frac{3 \hbar^{2}}{10 m_{e} e} \int d^{3} r \rho_{e, 1}(r)\left(3 \pi^{2} \rho_{e, 1}(r)\right)^{2 / 3} .
$$

Con esto, la energía total se evalua sujeta a las condiciones

$$
\frac{\partial E}{\partial \Lambda_{1}}=0,\left.\quad \frac{\partial E}{\partial N_{1}}\right|_{Z_{1}}=0 .
$$

La primera ecuación minimiza la energía respecto al parámetro $\Lambda$, la segunda condición, garantiza que el átomo neutro tiene menor energía que sus iones.

Para obtener resultados analíticos sin necesidad de recurrir a métodos numéricos, buscamos formas de $\Phi(x)$ analíticas. Para este fin escogemos una solución analítica aproximada para la ecuación de TF propuesta por Tietz [78]

$$
\Phi(x)=\frac{b^{2}}{(x+b)^{2}},
$$

donde $b=(8 / \pi)^{2 / 3}$ tal que la densidad electrónica está normalizada [78]. Sustituyendo la ec. (3.37) en (3.34), y siguiendo el procedimiento estandar [79] para el cálculo de la energía total [ec. (3.35)], obtenemos

$$
E=-\frac{Z_{1}^{7 / 3}}{c}\left(\frac{N_{1}}{Z_{1}}\right)^{1 / 3}\left(1-\frac{\lambda}{5} \frac{N_{1}}{Z_{1}}\right)^{2} \frac{e^{2}}{a_{0}}
$$




$$
\Lambda_{1}=\frac{c a_{0}}{Z_{1}^{1 / 3} b\left[1-(\lambda / 5)\left(N_{1} / Z_{1}\right)\right]}\left(\frac{N_{1}}{Z_{1}}\right)^{2 / 3}
$$

con $\lambda=\frac{5}{7}$ y $c=0.969376$.

Entonces de la ecuación de Poisson, podemos obtener el potencial electrónico, y usando la ec. (3.32), resolvemos para $x_{c}=r_{c} / \Lambda_{1}$, obteniendo

$$
x_{c}(v)=-2\left(\frac{b}{3}\right)+\frac{1}{g(a, b)}\left(\frac{b}{3}\right)^{2}+g(a, b)
$$

con

$$
g(a, b)=\left[\frac{a}{2}+\left(\frac{b}{3}\right)^{3}+\sqrt{\left(\frac{a}{2}\right)^{2}+a\left(\frac{b}{3}\right)^{3}}\right]^{1 / 3}
$$

donde $a(v)=b^{2} / 0.60647 y^{2}(v)$, y $y(v)=v /\left(v_{0} Z_{1}^{2 / 3}\right)$ es la velocidad efectiva del ion. $x_{c}(v)$ es la distancia radial, medida desde el nucleo del proyectil, para la cual la velocidad orbital de los electrones es igual a la velocidad del proyectil.

Finalmente sustituyendo las ecs. (3.34) y (3.37) en la ec. (3.33), obtenemos la siguiente relación para el número de electrones promedio retenidos por el proyectil a una velocidad $v$

$$
N_{1}(v)=Z_{1}\left(1-\frac{b^{2}\left[3 x_{c}(v)+b\right]}{\left[x_{c}(v)+b\right]^{3}}\right) .
$$

Esta expresión para el número de electrones retenidos por el proyectil, nos será útil para evaluar la sección de frenamiento y al igual que en la sección anterior, su discusión se dejara para la parte final de este capítulo. En la siguiente sección utilizamos este resultado para obtener una expresión analítica para la sección de frenamiento electrónico considerando el número de electrones retenidos por el proyectil.

\subsection{Expresión analítica para $S_{e}(v)$}

Para obtener una expresión analítica de la sección transversal de frenamiento electrónico $S_{e}(v)$ en la primera aproximación de Born, necesitamos los factores de forma atómico para el proyectil y blanco [vease ec. (3.29)]. Para estados con simetría esférica del proyectil y blanco [estado base $|0\rangle]$, la ec. (3.22) se reduce a

$$
{ }_{i} T_{00}(q)=4 \pi \int \frac{\rho_{e, i}(r) \sin (q r)}{q r} r^{2} d r, \quad i=1,2,
$$


donde $\rho_{e, i}(r)$ es la densidad electrónica del estado base. Empleando la densidad de $\mathrm{TF}$ [ ecs. (3.34) y (3.37)] en la ec. (3.43) obtenemos

$$
{ }_{i} T_{00}(q)=N_{e, i}\left[1-\left(q \Lambda_{i} b\right)^{2} g\left(q \Lambda_{i} b\right)\right],
$$

donde

$$
g(z)=-\cos (z) C i(z)-\sin (z) \operatorname{si}(z)
$$

es la función auxiliar para las funciones integrales seno y coseno [80]. Usando la representación analítica para $g(z)$ [81],

$$
g(z)=\frac{0.37093}{z^{2}+13.88129}+\frac{0.62907}{z^{2}+0.967375},
$$

obtenemos una expresión analítica para los factores de forma atómico.

Finalmente, sustituyendo las ecs. (3.44) y (3.46) en la ec. (3.29), encontramos que la contribución a la sección transversal de frenamiento electrónico debido a las excitaciones en la estructura electrónica del proyectil y del blanco es

$$
\begin{aligned}
S_{e, i}(v)= & \frac{4 \pi e^{4}}{m_{e} v^{2}} N_{i} Z_{j}^{2}\left\{f_{i}^{2} \ln \left(\frac{q_{\max }}{\bar{q}_{\min }}\right)\right. \\
& +\left(1-f_{i}\right)\left[A\left[f_{i}+B\right] \ln \left(\frac{\left(q_{\max } \Lambda_{j}\right)^{2}+C}{\left(\bar{q}_{\min } \Lambda_{j}\right)^{2}+C}\right)\right. \\
& \left.+D\left[f_{i}+E\right] \ln \left(\frac{\left(q_{\max } \Lambda_{j}\right)^{2}+F}{\left(\bar{q}_{\min } \Lambda_{j}\right)^{2}+F}\right)\right] \\
& +\left(1-f_{i}\right)^{2}\left(G\left[\frac{1}{\left(q_{\max } \Lambda_{j}\right)^{2}+C}-\frac{1}{\left(\bar{q}_{\min } \Lambda_{j}\right)^{2}+C}\right]\right. \\
& \left.\left.+H\left[\frac{1}{\left(q_{\max } \Lambda_{j}\right)^{2}+F}-\frac{1}{\left(\bar{q}_{\min } \Lambda_{j}\right)^{2}+F}\right]\right)\right\},
\end{aligned}
$$

$\operatorname{con} q_{\max }=2 m v / \hbar, \bar{q}_{\min }=\epsilon / \hbar v$.

La fracción de ionización [60] $f_{i}$ esta definida como $f_{i}=1-N_{i} / Z_{i}$ y el subindice $i$ representa el blanco (o proyectil) y $j$ el proyectil (o blanco) respectivamente. Notemos que sólo $N_{1}$ depende de la velocidad del proyectil mediante la ec. (3.42). 
Aquí

$$
\begin{aligned}
& A=0.448685 \\
& B=0.402031 \\
& C=0.278190 \\
& D=0.051315 \\
& E=6.228480 \\
& F=3.991870 \\
& G=0.055044 \\
& H=0.274619
\end{aligned}
$$

Estas expresiones se pueden aplicar inmediatamente, y junto con la ec. (3.42) para el número de electrones retenidos por el proyectil en función de $v$, nos da la sección de frenamiento electrónico calculada a partir de $Z_{1}, N_{2}$, y las energías medias de excitación $I_{i}$, las cuales se pueden obtener teórica o experimentalmente (e. g. [82]).

\subsection{Análisis y discusión}

Analicemos el resultado final para la sección de frenamiento electrónico en la primera aproximación de Born, [ec. (3.29)] en algunos casos límite de interés para relacionar esta teoría general a algunos casos especiales bién conocidos. Si consideramos un proyectil desnudo con $N_{1}=0$ y carga $Z_{1}$ colisionando con un blanco con energía media de excitación $I_{2}$, entonces de la ecuación (3.31) $\alpha=0$, and $\epsilon=I_{2}$, tal que la ecuación (3.29) nos da

$$
S_{e}(v)=\frac{4 \pi e^{4}}{m_{e} v^{2}} Z_{1}^{2} N_{2} \ln \left(\frac{2 m_{e} v^{2}}{I_{2}}\right),
$$

la cual es el resultado estandard de Bethe en la primera aproximación de Born. Para el caso inverso, un proyectil con $N_{1}$ electrones ligados colisionando con un blanco completamente ionizado $\left(N_{2}=0\right), \alpha=\infty$ y $\epsilon=I_{1}$, y tal que de la ec. (3.29) obtenemos

$$
S_{e}(v)=\frac{4 \pi e^{4}}{m_{e} v^{2}} Z_{2}^{2} N_{1} \ln \left(\frac{2 m_{e} v^{2}}{I_{1}}\right) .
$$


Esto es, sin embargo, el resultado estándar en la teoría de Bethe para un proyectil desnudo de carga $Z_{2}$ incidente en un blanco con $N_{1}$ electrones y energía media de excitación dada por $I_{1}$, i.e. , el proyectil vería a los blancos como los proyectiles frenándose. Los mismos resultados se obtienen de la ec. (3.47) bajo las mismas suposiciones.

Ahora consideremos el caso especial en el que no se consideran excitaciones en el proyectil, i.e. consideramos sólo procesos elásticos sobre el proyectil $\left(n=n_{0}\right)$. Con ésto, en la ec. (3.23) la contribución de la estructura electrónica del proyectil desaparece y obtenemos

$$
S_{e}(v)=\frac{2 e^{4}}{m_{e} v^{2}} \sum_{m} \int_{w_{m_{0} m} / v}^{2 m_{e} v / \hbar}\left[Z_{1}-{ }_{1} T_{n_{0} n_{0}}(\mathbf{q})\right]_{2}^{2}{ }_{2} F_{m_{0} m}(\mathbf{q}) \frac{d \mathbf{q}}{q^{2}},
$$

donde ${ }_{1} T_{n_{0} n_{0}}$ es el factor de forma atómico para el proyectil [ec. (3.22)]. Esta ecuación ha sido aplicada recientemente al proceso de frenamiento de iones con estructura electrónica sin excitaciones [57]. En este límite, y aplicando las mismas suposiciones para derivar la ec. (3.29), encontramos que

$$
S_{e}(v)=\frac{2 e^{4}}{m_{e} v^{2}} N_{2} \int_{I_{2} / v \hbar}^{2 m_{e} v / \hbar}\left[Z_{1}-{ }_{1} T_{n_{0} n_{0}}(\mathbf{q})\right]^{2} \frac{d \mathbf{q}}{q^{2}} .
$$

Sin embargo,

$$
\rho_{n e, 1}(\mathbf{q})=e\left[Z_{1}-{ }_{1} T_{n_{0} n_{0}}(\mathbf{q})\right]
$$

es la transformada de Fourier de la densidad de carga total (electrónica + nuclear) del proyectil con carga nuclear $Z_{1}$ moviendose con $N_{1}$ electrones ligados. Por lo que,

$$
S_{e}(v)=\frac{2 e^{2}}{m_{e} v^{2}} N_{2} \int_{I_{2} / v \hbar}^{2 m_{e} v / \hbar}\left|\rho_{n e, 1}(\mathbf{q})\right|^{2} \frac{d \mathbf{q}}{q^{2}} .
$$

Notando que un gas de electrones de densidad $n_{2} N_{2}$ tiene una frecuencia de plasma $\omega_{0}$ dada por

$$
\omega_{0}^{2}=\frac{4 \pi n_{2} e^{2}}{m_{e}} N_{2},
$$

la ec. (3.54) puede escribirse, para simetría esférica en la forma

$$
S_{e}(v)=\frac{\omega_{0}^{2}}{n_{2} v^{2}} \int_{I_{2} / v \hbar}^{2 m_{e} v / \hbar}\left|\rho_{n e, 1}(q)\right|^{2} \frac{d q}{q} .
$$




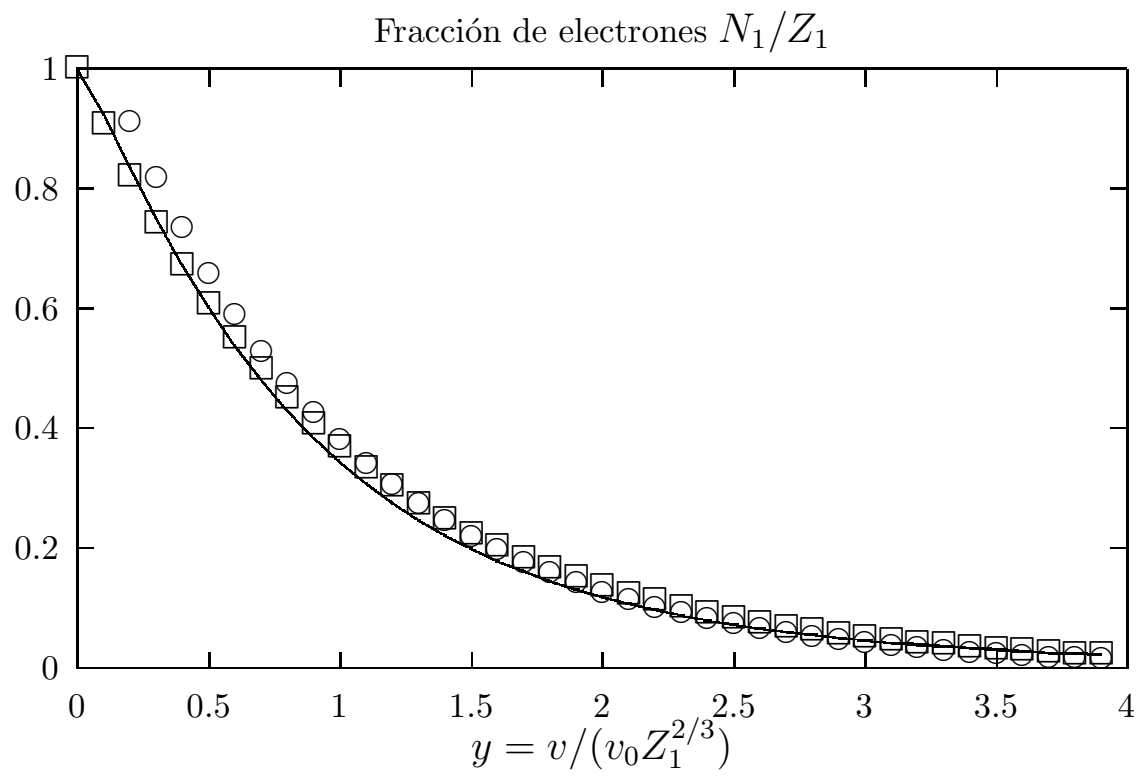

Figura 3.1: Fracción de electrones que retiene el proyectil $N_{1} / Z_{1}$ [vease la ec. (3.42)] como función de la velocidad de el proyectil. Línea contínua, este trabajo; o, Anthony and Landford [83]; $\square$, Betz et al. [84].

Esta ecuación tiene precisamente la misma estructura que la usada en el análisis del poder de frenamiento de sólidos en la aproximación de respuesta dieléctrica [60] para grandes velocidades del proyectil.

\subsubsection{La precisión de la suposición adiabática}

En ésta sección analizaremos los resultados obtenidos con los métodos sugeridos en las secciones anteriores, para determinar el número de electrones en el proyectil como función de su velocidad [ver ec. (3.42)] así como el comportamiento de los factores de forma atómicos calculados en la ec. (3.44).

En la figura 3.1 mostramos la fracción de electrones que permanecen en el proyectil $N_{1}(v) / Z_{1}$ como función de la velocidad efectiva del proyectil $y(v)=v /\left(v_{0} Z_{1}^{2 / 3}\right)$ comparando con los valores experimentales de Anthony y Lanford [83] y los de Betz et al. [84] para un blanco de Aluminio. Como una característica del modelo de TF, este no representa el carácter individual de cada átomo (por ejemplo, estructura de capas) y la ec. (3.42) nos da una representación universal como función de la velocidad efectiva del proyectil. Además, debemos tener en cuenta que los valores experimentales mostrados aquí, son resultado del uso del concepto de "carga efec- 


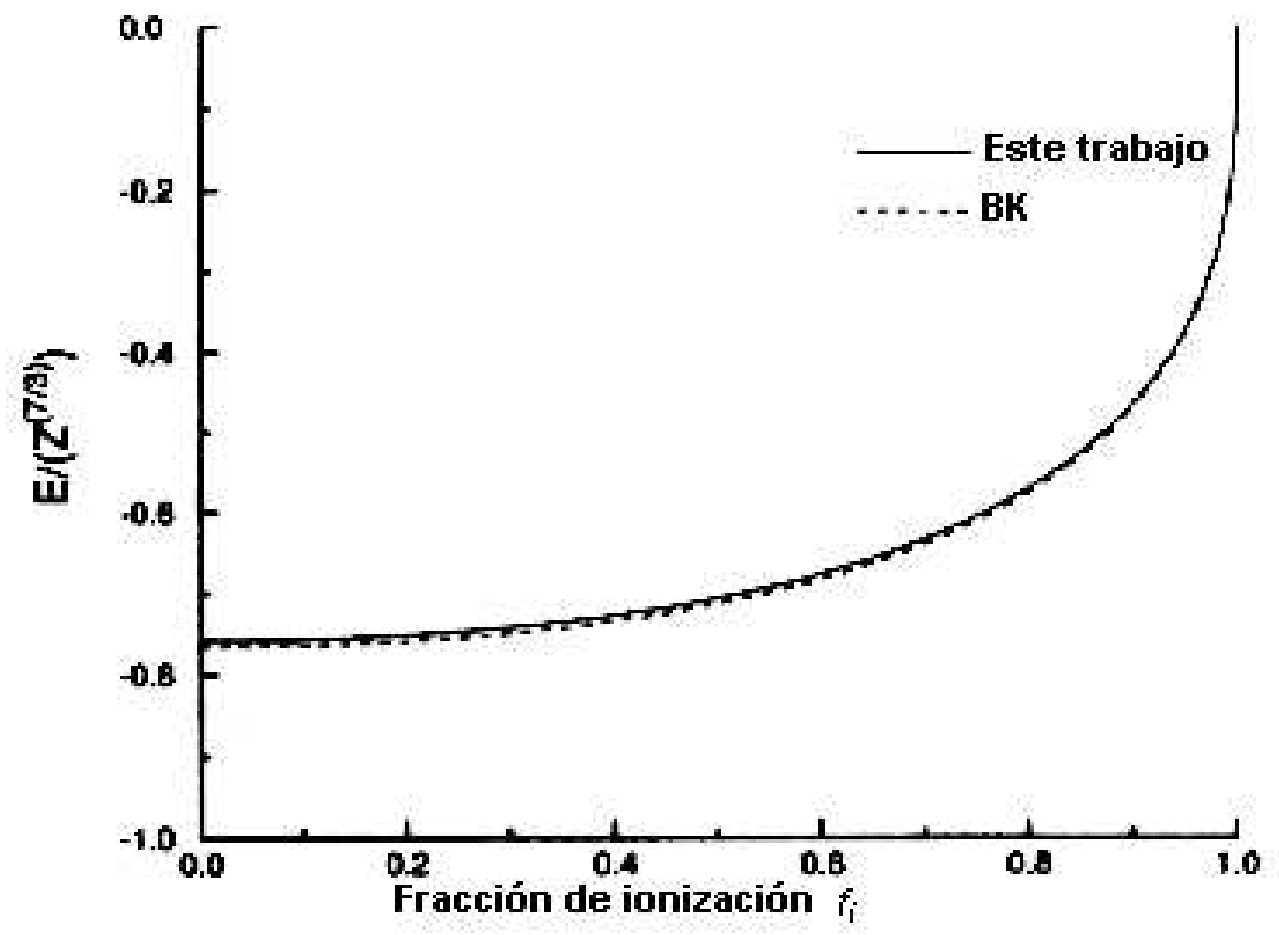

Figura 3.2: Comparación de la energía total de elion como función de la fracción de ionización $\left(f_{i}=1-N_{1} / Z_{1}\right)$, calculada en éste modelo y en el modelo de Brandt y Kitagawa [60]. Línea contínua, este modelo; - - - - modelo BK.

tiva" [83], el cual es un poco diferente del concepto de carga promedio de el ion [27] usado aquí. Esta diferencia es apreciable en la región de bajas velocidades de la figura 3.1 donde los procesos de pérdida y captura de electrones en el proyectil como función del tipo de blanco deben de tomarse en cuenta en una forma más precisa [27]. Sin embargo, para altas velocidades, el criterio de Bohr tiene una mayor probabilidad de ser válido, y encontramos que el número de electrones retenidos por el proyectil está en buen acuerdo con los resultados experimentales.

Además, encontramos que nuestro modelo para $\rho_{e, 1}(r)$ se comporta bien cuando se compara con el modelo de Lenz-Jensen (LJ) [85] o el modelo de Brandt-Kitagawa (BK) [60]. Por ejemplo, como se muestra en la figura 3.2, la energía [ec. (3.38)] para diferentes grados de ionización, $f_{i}=1-N_{1} / Z_{1}$ calculada con nuestro modelo y el modelo BK están en muy buén acuerdo entre una y otra. Con ésto, confiamos en 


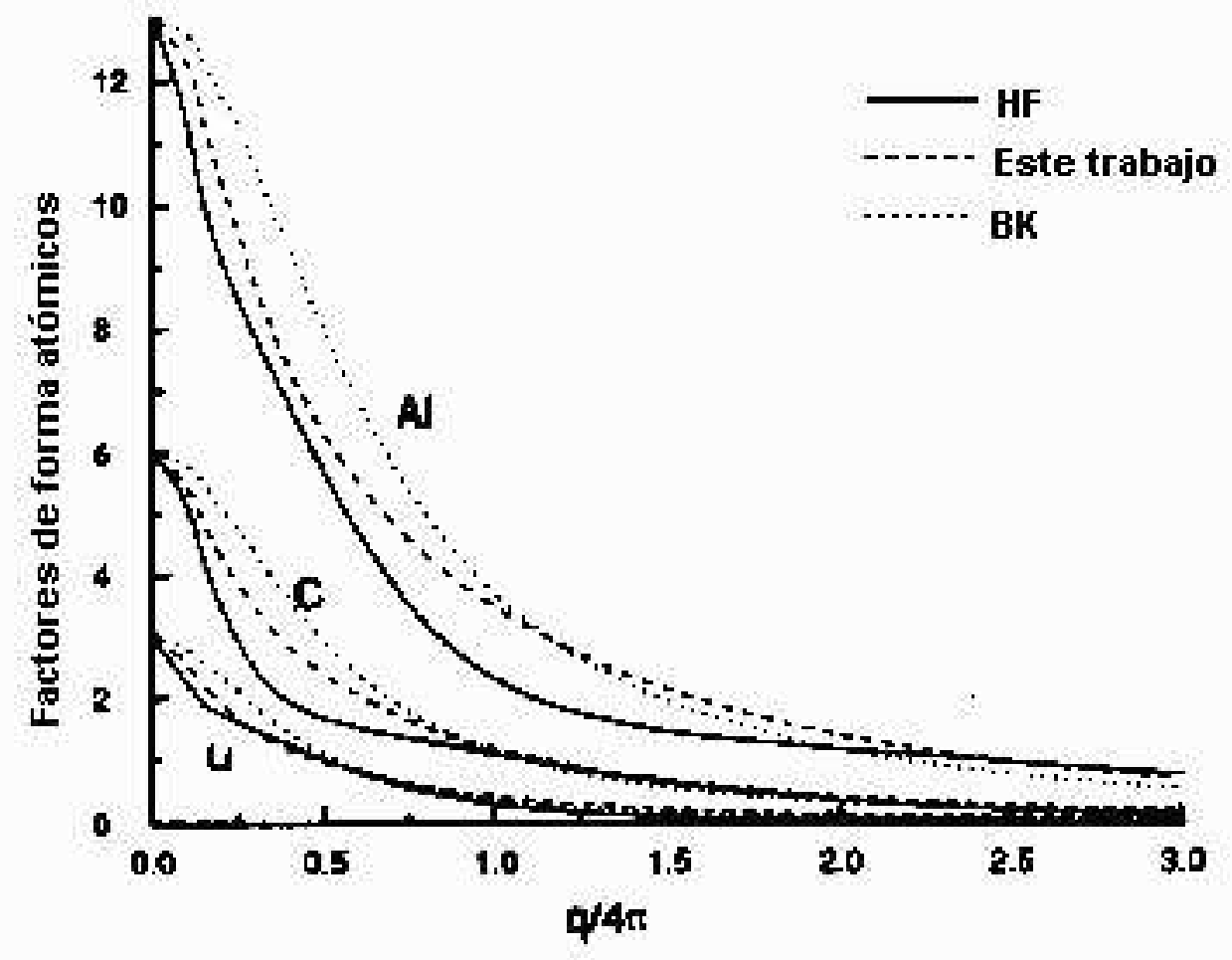

Figura 3.3: Comparación de los factores de forma para átomos neutros como función del momento transferido q. Línea continua, cálculos HF [86]; ‥ modelo BK [60]; - - - - éste trabajo.

que los cálculo de $N_{1}$ llevados a cabo con ésta aproximación son los suficientemente precisos como para calcular el poder de frenamiento de un ion con electrones ligados.

En la figura 3.3 mostramos los factores de forma atómicos para varios átomos neutros $\left(N_{1}=Z_{1}\right)$ representativos comparados con los resultados Hartree-Fock de Hubbel et al. [86] y con la teoría de BK [60]. Como vemos, nuestro modelo da un mejor acuerdo con el modelo HF que el sugerido por la teoría BK. Esto es debido probablemente al hecho de que nuestro modelo está forzado a satisfacer la teoría de TF.

\subsubsection{Comparación con el experimento}

Como un ejemplo, calculamos la sección transversal de frenamiento electrónico para proyectiles de $\mathrm{He}$, Li, y $\mathrm{B}$ incidentes en blancos de $\mathrm{C}$ y $\mathrm{Al}$ y comparamos con los 


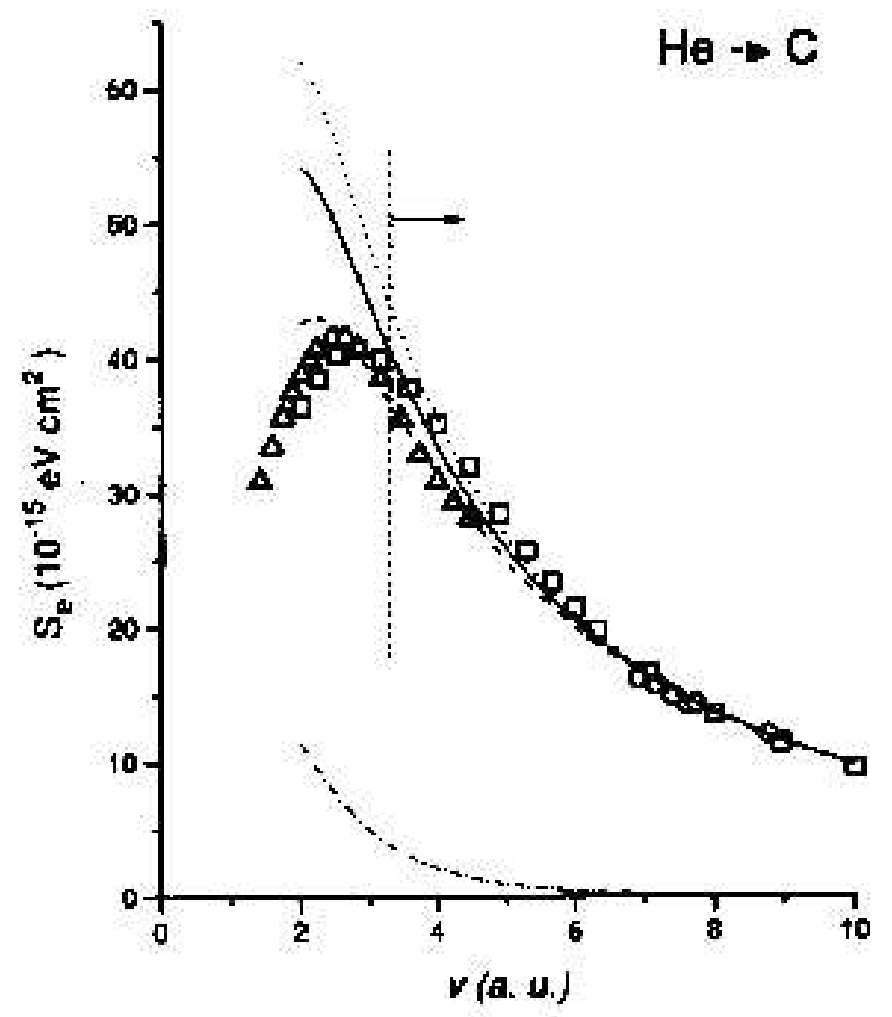

Figura 3.4: Comparación de la sección de frenamiento electrónico para He incidente en $\mathrm{C}$ con resultados experimentales. - . - - - representa la contribución de la estructura electrónica de el proyectil. - - - representa la contribución del blanco; - - - es la contribución total y $\cdots$ representa la contribución logarítmica estandard de Bethe. El ámbito de aplicabilidad de la teoría está en región de altas velocidades a la derecha de la linea vertical. Los símbolos representan los datos experimentales de: [87] $(\square),[88](\triangle)$ y [89] (०).

datos experimentales disponibles [87]-[92]. Como los blancos son neutros, la fracción de ionización $f_{i}=0$ y de aquí $\epsilon=I_{2}$. La energía media de excitación para $\mathrm{C}$ y $\mathrm{Al}$ neutros es $I_{2}=73.8$ y $I_{2}=160.1 \mathrm{eV}$, respectivamente [82]. La energía de excitación para los proyectiles, como lo requiere la ec. (3.30), no se necesita en estos cálculos puesto que los blancos son neutros.

En la figura 3.4 comparamos la sección de frenamiento electrónico para proyectiles de He incidentes en $\mathrm{C}$ con los resultados experimentales de Northcliffe y Schilling [87] y los de Santry y Werner [88, 89]. Para la región de altas veloci- 


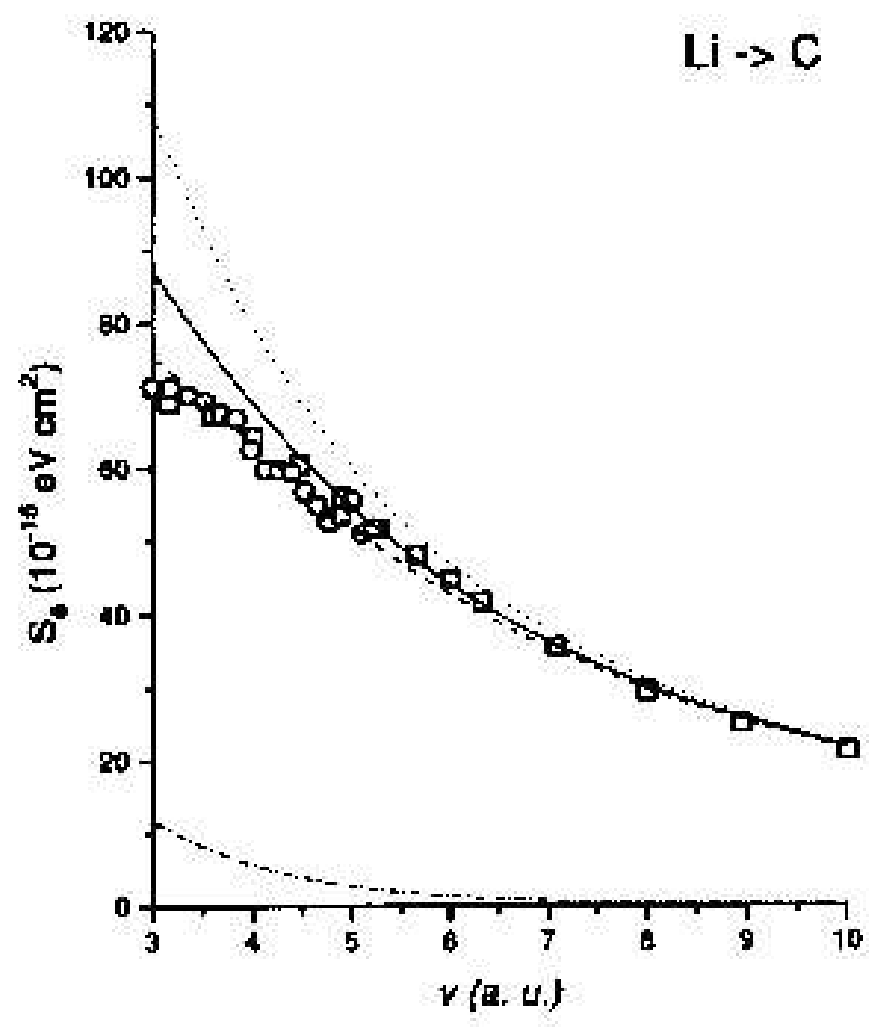

Figura 3.5: Comparación de la sección de frenamiento electrónico de Li sobre C neutro con los resultados experimentales. Las etiquetas son las mismas que en la figura 3.4. Los datos experimentales son tomados de la referencias [87] (Box), y [91] (०).

dades (delimitada por la linea vertical) en donde se puede aplicar esta teoría, encontramos que hay buén acuerdo con el experimento. En este caso tenemos un ion ligero incidiendo en un blanco ligero, y la condición $v>v_{0}$ se satisface.

En la figura 3.5 mostramos la sección transversal de frenamiento electrónico para un proyectil de Li incidente en $\mathrm{C}$, comparando con los datos experimentales de Northcliffe y Schilling [87] y los de Lin et al. [91]. De nuevo, en la región de validez de esta teoría, encontramos un buen acuerdo con los resultados experimentales.

Por otra parte, en la figura 3.6 comparamos los resultados obtenidos para proyectiles de $\mathrm{Li}$ incidentes en $\mathrm{Al}$ con los datos experimentales de Northcliffe y Schilling [87], Andersen et al. [90], y los de Lin et al. [91].

De la misma manera en la figura 3.7 comparamos los resultados para proyectiles 


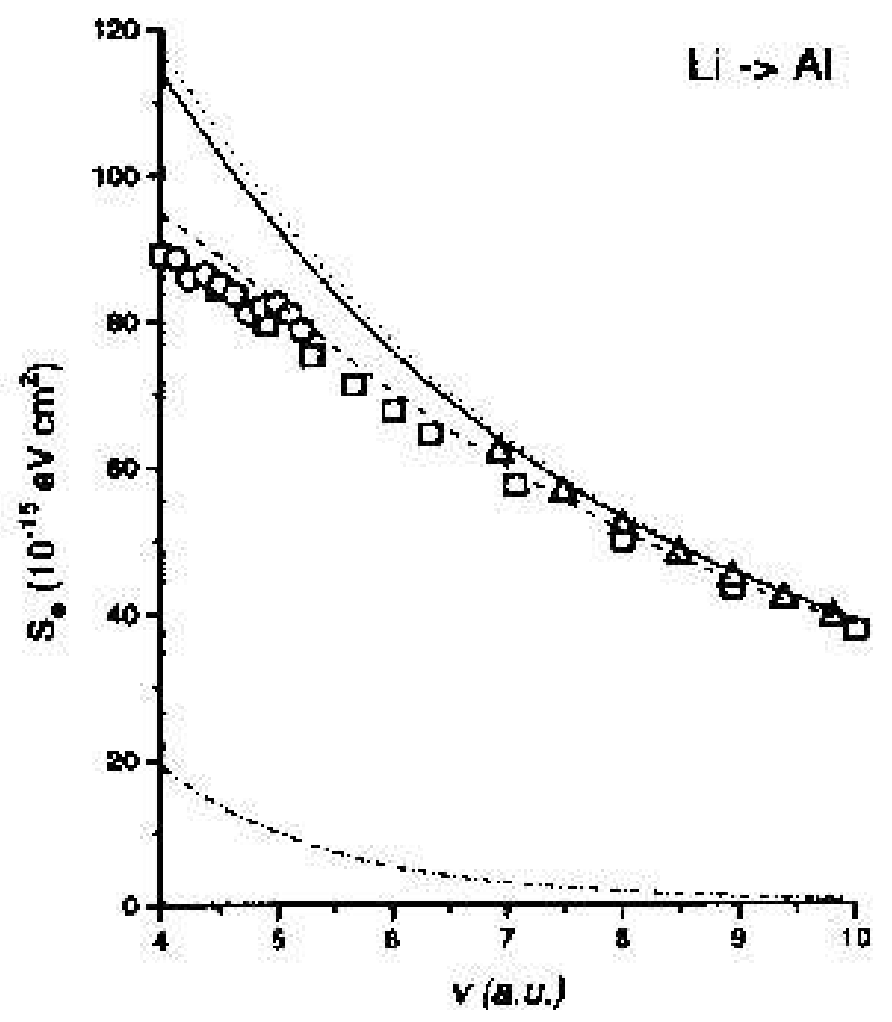

Figura 3.6: Comparación de la sección de frenamiento electrónico de Li sobre Al neutro con los resultados experimentales. Las etiquetas son las mismas que en la figura 3.4. Los datos experimentales son tomados de la referencias [87] (Box), [90] $(\triangle), y[91](0)$.

de B incedentes en C con los resultados experimentales de Booth and Grant [92]. En estos dos casos vemos que para velocidades del proyectil cercanas a el máximo de $S_{e}(v)$, hay una sobre-estimación de la sección de frenamiento electrónico $S_{e}(v)$ para éstos proyectiles pesados incidentes en blancos pesados. En ambos casos la velocidad orbital de los electrones del proyectil y blanco es mayor que la velocidad del proyectil, de aquí es necesario considerar corrección a la aproximación de Bethe para las regiones de bajas velocidades. Las correcciones más importantes son las correcciones por capas y las correcciones de Barkas. Ambas son negativas lo cual mejorara los resultados obtenidos con respecto al experimento. Las correcciones más pequeñas son las de Bloch y tienen signo positivo.

Además, en las mismas figuras graficamos el término estandard de Bethe [ec. 


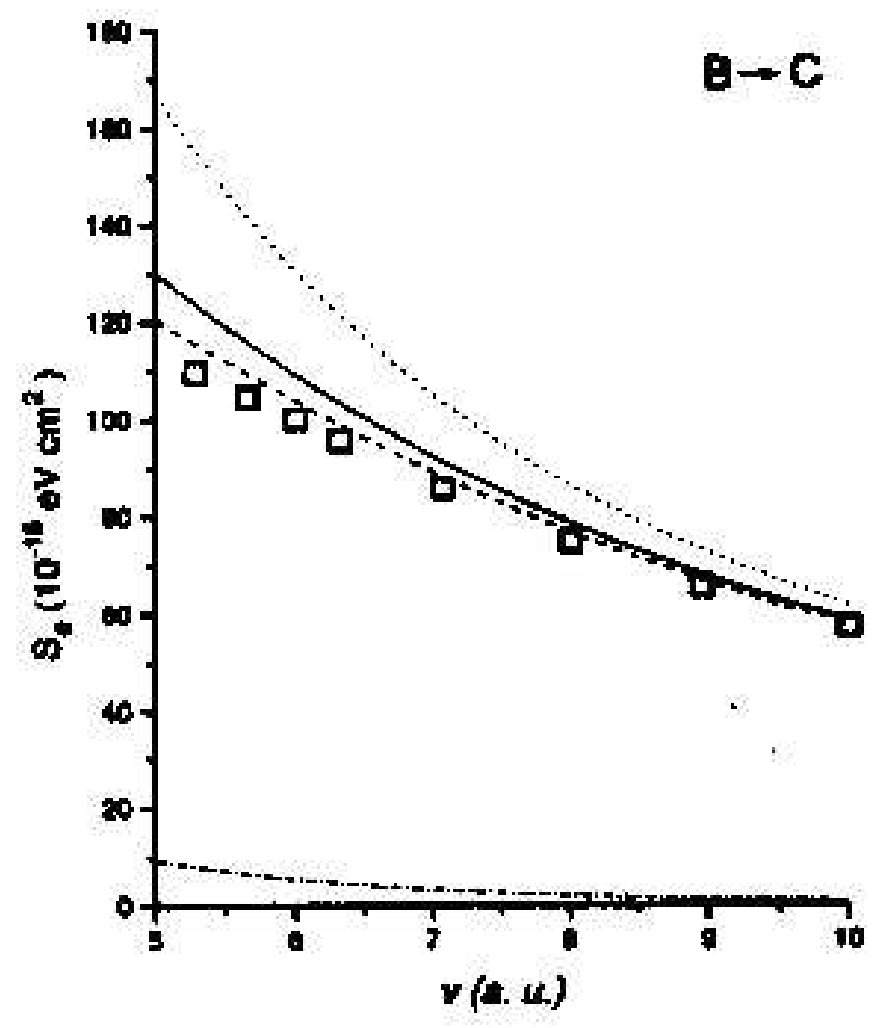

Figura 3.7: Comparación de la sección de frenamiento electrónico de B sobre C neutro con los resultados experimentales. Las etiquetas son las mismas que en la figura 3.4. Los datos experimentales son tomados de la referencias [92].

(A.18)] para un proyectil sin estructura electrónica, y ademas mostramos las contribuciones individuales debido a la estructura del proyectil y del blanco. Es interesante notar el orden de magnitud de la contribución de proyectil $S_{e, 1}$ a $S_{e}$, la cual es del orden de 10-20\%, en la parte correspondiente a bajas velocidades donde éste modelo es aplicable. Esto se puede observar en todos los casos que hemos tratado. Este comportamiento es un reflejo del número de electrones ligados al proyectil como función de la velocidad del proyectil, puesto que $S_{e, 1} \propto N_{1}$, (ver figura 3.1). Lo mismo se ha encontrado en otras teorías de frenamiento a bajas velocidades, como por ejemplo la teoría de Firsov [93], donde la contribución a $S_{e}(v)$ es una suma de las contribuciones del la estructura electrónica del proyectil más la contribución de la estructura electrónica del blanco [94]. 
48 CAPÍTULO 3. TEORÍA DE BETHE: PROYECTILES CON ESTRUCTURA

\subsection{Conclusiones}

Hemos derivado una expresión para el poder de frenamiento electrónico para iones rápidos con $N_{1}(v)$ electrones ligados incidentes en un blanco iónico tomando en cuenta las excitaciones e ionizaciones de los electrones ligados en el proyectil y blanco. La fórmula se deriva del mismo conjunto de suposiciones que la fórmula estandard de Bethe para el poder de frenamiento electrónico en la primera aproximación de Born. La fórmula general requiere conocer el espectro total de excitación tanto del proyectil como del blanco, y por tanto determinar las Tensiones de Oscilador Generalizado (GOS). Debido a las dificultades para evaluar esas cantidades, hemos derivado una expresión aproximada usando la teoría de Thomas-Fermi, obteniendo expresiones analíticas para todas las cantidades claves de la teoría. La distribución de electrones en el proyectil se calculó a partir del criterio adiabático de Bohr y la descipción de Thomas-Fermi del átomo, conduciéndonos a una expresión analítica para la fracción de ionización del proyectil, $f_{i}$. Finalmente el poder de frenamiento total se pudo escribir como la suma de las contribuciones de la estructura electrónica del proyectil y del blanco, encontrando que la contribución del proyectil es importante a velocidades intermedias.

Dada la importancia que tiene el conocer los GOS, en el capítulo siguiente analizamos la regla de suma de Bethe para el caso de una aproximación para los orbitales que describen el sistema. 


\section{Capítulo 4}

\section{Regla de suma de Bethe en la aproximación RPA}

\subsection{Resumen}

En éste capítulo nos concentramos en describir la regla de suma de Bethe (BSR) dentro de la aproximación de fase aleatoria (RPA), la cual, como consecuencia de la imposibilidad de cálcular el espectro completo de excitación para un sistema dado, resulta ser una de las aproximaciones más recurridas en química cuántica. Principalmente nos concentraremos en demostrar que la BSR se satisface en esta aproximación.

\subsection{Introducción}

Como ya hemos visto dentro de la teoría de Bethe, las colisiones inelásticas de partículas cargadas rápidas con la materia están completamente descritas por la distribución de las Tensiones de Oscilador Generalizado (GOS) que caracterizan la colisión. Estas cantidades, que describen las excitaciones de los $\mathrm{N}$ electrones del blanco o del proyectil [ver ec. (3.24)] desde un estado inicial $|0\rangle$ a un estado final $|n\rangle$ pueden escribirse como

$$
F_{n 0}(\mathbf{q})=\frac{2 m_{e} E_{n o}}{\hbar^{2} q^{2}}\left|M_{n 0}(\mathbf{q})\right|^{2}
$$


en la cual $E_{n 0}$ es la energía requerida para una transición desde $|0\rangle$ hasta $|n\rangle$, q es el momento transferido y

$$
M_{n 0}(\mathbf{q})=\left\langle n\left|\sum_{j=1}^{N} e^{-i \mathbf{q} \cdot \mathbf{r}}\right| 0\right\rangle
$$

es el factor de forma inelástico de la dispersión el cuál describe la dinámica de los $\mathrm{N}$ electrones del átomo blanco o molécula. Si graficamos los GOS como una función de la energía y momento transferido, generamos una superficie denominada la superficie de Bethe para el blanco, y es esta superficie, la que “... contiene toda la información respecto a la dispersión inelástica de las partículas cargadas por un átomo o molécula dentro de la primera aproximación de Born" [73].

De tal manera que para poder calcular la pérdida de energía para blancos atómicos o moleculares con suficiente confiabilidad, uno debe escoger una base apropiada para representar al estado $|n\rangle$ en el cálculo de los GOS. Así, necesitamos describir un procedimiento factible en la determinación adecuada de las funciones de base.

La manera de determinar si una base es lo suficientemente adecuada para describir cierta propiedad es mediante el uso de la regla de suma de Bethe (BSR) [ec. (3.26)], tal que la suma sobre todo el espectro de excitación debe ser igual al número de electrones en el sistema. En un cálculo realista para un sistema de muchos electrones, la función de onda es aproximada, por lo tanto, la pregunta que surge es si la BSR es válida al emplear una representación aproximada para el estado $|n\rangle$, tal que se pueda aplicar un procedimiento de química cuántica al cálculo de la sección de frenamiento.

La manera más común de obtener una base es via optimización de la energía. Sin embargo es bién sabido que la optimización de bases para una propiedad en particular no es siempre buena en el cálculo de alguna otra, quizas sólo si está relacionada de alguna manera a ésta. Esto nos conduce a tener algún otro criterio para determinar la base en conexión con alguna propiedad en particular.

Para altas velocidades del proyectil comparadas con la velocidad electrónica de los electrones del blanco, la aproximación de Bethe nos conduce a la ec. (A.18) en donde la energía media de excitación $I_{2}$ esta dada por la ec. (A.16) en términos de las Tensiones de Oscilador Dipolar (DOS) $\left\{f_{0 n}\right\}$ [ver ec. (A.14)]. Así, una 
cantidad usada para la determinación de la calidad de una base es la regla de suma de Thomas-Reiche-Kuhn (TRK) [95, 96]

$$
\sum_{n>0} f_{0 n}=N_{2}
$$

la cual nos dice que para una base completa, las Tensiones de Oscilador Dipolar sumados sobre todas las excitaciones nos dan el número de electrones del blanco. Sin embargo, los conjuntos de bases computacionales usados en Química Cuántica, no son completos y así el problema se ha resuelto exactamente en pocas ocasiones [97]. Por otro lado, dentro de las aproximaciones usadas en Química Cuántica, se ha demostrado que para una base completa, la regla de suma TRK se satisface dentro de la aproximación de fase aleatoria (RPA) [98]-[101], removiéndose así una de las restricciones en la determinación de una buena base. Con ésto, la regla de suma TRK en la aproximación RPA ha sido utilizada frecuentemente como un criterio para determinar una buena base en este tipo de cálculos [48]. Debemos notar sin embargo, que el cumplimiento de la regla de suma TRK es una condición necesaria pero no suficiente para la completez de la base.

Con estos antecedentes, surge la necesidad de investigar si en la aproximación RPA, se puede generalizar la regla de suma para cualquier transferencia de momento (regla de suma de Bethe, BSR). Así, antes de demostrar la versión general, revisemos algunos resultados que necesitaremos para nuestro fín.

\subsection{Las ecuaciones de Hartree-Fock}

La función de onda normalizada de Hartree-Fock (HF) [102] para el estado base $\Phi_{0}$ está dada por el determinante de Slater para N-electrones

$$
\Phi_{0}=\frac{1}{n !^{1 / 2}} \operatorname{det}\left|\phi_{a}(1) \phi_{b}(2) \ldots \phi_{z}(N)\right|
$$

y la energía del estado base está dada por el valor de expectación

$$
E=\left\langle\Phi_{0}|\hat{H}| \Phi_{0}\right\rangle
$$

donde $\hat{H}$ es el Hamiltoniano que describe al átomo blanco y que esta dado por

$$
\hat{H}=\sum_{i}^{N} h_{i}+\frac{1}{2} \sum_{i \neq j}\left(\frac{e^{2}}{\left|\mathbf{r}_{i}-\mathbf{r}_{j}\right|}\right)
$$


y donde $h_{i}$ es un Hamiltoniano hidrogenoide para el $i$-ésimo electrón en el campo de un nucleo desnudo de carga $Z e$. Nosotros buscamos un conjunto de orbitales $\phi$ que minimicen la energía $E$ : esta condición nos conduce a las ecuaciones de HF.

Como un primer paso, derivemos una expresión para $E$ en términos de los orbitales. De la ec. (4.5) y (4.6) obtenemos

$$
E=\left\langle\Phi_{0}\left|\sum_{i}^{N} h_{i}+\frac{1}{2} \sum_{i \neq j}\left(\frac{e^{2}}{\left|\mathbf{r}_{i}-\mathbf{r}_{j}\right|}\right)\right| \Phi_{0}\right\rangle .
$$

Para el primer término podemos escribir

$$
\left\langle\Phi_{0}\left|\sum_{i} h_{i}\right| \Phi_{0}\right\rangle=\left\langle\Phi_{0}\left|h_{1}+h_{2}+\cdots+h_{N}\right| \Phi_{0}\right\rangle=\sum_{i}\left\langle\phi_{i}(1)\left|h_{1}\right| \phi_{1}(1)\right\rangle .
$$

La segunda igualdad sigue del hecho de que los orbitales son ortonormales.

La expansión de $\Phi_{0}$ en términos de los orbitales $\phi_{a}$ [ver ec. (4.4)] nos reducen el segundo término de $\hat{H}$ en

$$
\frac{1}{2} \sum_{i \neq j} \int \phi_{i}^{*}(1) \phi_{j}^{*}(2)\left(\frac{e^{2}}{r_{12}}\right)\left\{\phi_{i}(1) \phi_{j}(2)-\phi_{j}(1) \phi_{i}(2)\right\} d^{3} x_{1} d^{3} x_{2} .
$$

Entonces, con la definición

$$
\left\langle\phi_{a} \phi_{b} \mid \phi_{c} \phi_{d}\right\rangle=\int \phi_{a}^{*}(1) \phi_{b}^{*}(2)\left(\frac{e^{2}}{r_{12}}\right) \phi_{c}(1) \phi_{d}(2) d^{3} x_{1} d^{3} x_{2}
$$

la ecuación para la energía de HF se reduce a

$$
E=\sum_{i=1}^{N}\left\langle\phi_{i}|h| \phi_{i}\right\rangle+\frac{1}{2} \sum_{i, j}^{N}\left\{\left\langle\phi_{i} \phi_{j} \mid \phi_{i} \phi_{j}\right\rangle-\left\langle\phi_{i} \phi_{j} \mid \phi_{j} \phi_{i}\right\rangle\right\} .
$$

Notemos que no se ha excluido el término $i=j$ en la segunda suma debido a que éste es identicamente cero. La ec. (4.11) es una expresión para la energía como función de los orbitales, ésto es, para cada función $\phi$ esta asociado un sólo valor de $E_{0}$.

Para derivar las ecuaciones de HF usaremos la técnica de variación funcional. La energía $E$ es una función del determinante de Slater $\Phi$. Para encontrar el determinante particular $\Phi$ para el cual $E$ es mínima, i.e. $\Phi_{0}$, encontramos $\Phi$ para la cual un pequeño cambio $\Phi \rightarrow \Phi+\delta \Phi$ no produce cambios en el valor de $E$ a primer orden en $\delta \Phi$. Para un cambio infinitesimal $\delta \Phi$, tenemos

$$
E(\Phi+\delta \Phi)=\langle\Phi+\delta \Phi|\hat{H}| \Phi+\delta \Phi\rangle=\langle\Phi|\hat{H}| \Phi\rangle+\delta\langle\Phi|\hat{H}| \Phi\rangle
$$


donde

$$
\delta\langle\Phi|\hat{H}| \Phi\rangle=\langle\delta \Phi|\hat{H}| \Phi\rangle+\langle\Phi|\hat{H}| \delta \Phi\rangle=\delta E .
$$

Buscamos el determinante $\Phi$ para el cual $\delta E=0$. Para ésto, usamos la expresión para $E$ dada por la ec. (4.11), sin embargo, tenemos una restricción adicional de que los orbitales deben ser ortonormales, con lo cual debemos utilizar la técnica de los multiplicadores indeterminados de Lagrange.

Debemos satisfacer la condición

$$
\int \phi_{i}^{*}(1) \phi_{j}(1) d^{3} x_{1}=\delta_{i j}
$$

donde $\delta_{i j}$ es la delta de Kronecker, la restricción es entonces de la forma

$$
g=\sum_{i, j=1}^{N}\left\{\left\langle\phi_{i} \mid \phi_{j}\right\rangle-\delta_{i j}\right\}=0
$$

que cuando los orbitales son cambiados por una cantidad infinitesimal $\delta \phi$ se modifica a

$$
\delta g=\sum_{i, j=1}^{N}\left\{\left\langle\delta \phi_{i} \mid \phi_{j}\right\rangle+\left\langle\phi_{i} \mid \delta \phi_{j}\right\rangle\right\}
$$

puesto que $\delta_{i j}$ es constante.

En este punto tomamos en cuenta las restricciones introduciendo los multiplicadores indeterminados $\epsilon_{j i}$ y entonces buscar la condición para la cual

$$
\delta E-\sum_{i, j=1}^{N} \epsilon_{j i}\left\{\left\langle\delta \phi_{i} \mid \phi_{j}\right\rangle+\left\langle\phi_{i} \mid \delta \phi_{j}\right\rangle\right\}=0 .
$$

Haciendo la variación en la ec. (4.11), substituyendo en la ec. (4.17) y factorizando el término $\delta \phi_{i}^{*}$ obtenemos que

$$
\sum_{i=1}^{N} \int \delta \phi_{i}^{*}(1)\left(h_{1} \phi_{i}(1)+\sum_{j=1}^{N}\left\{J_{j}(1) \phi_{i}(1)-K_{j}(1) \phi_{i}(1)-\epsilon_{j i} \phi_{j}(1)\right\}\right) d^{3} x_{1}+c c .=0
$$

donde $c c$. implica complejo conjugado, $J$ y $K$ son los operadores de Coulomb e intercambio definidos por

$$
\begin{aligned}
J_{u}(1) \phi_{a}(1) & =\left\{\int \phi_{u}^{*}(2) \frac{e^{2}}{r_{12}} \phi_{u}(2) d^{3} x_{2}\right\} \phi_{a}(1) \\
K_{u}(1) \phi_{a}(1) & =\left\{\int \phi_{u}^{*}(2)\left(\frac{e^{2}}{r_{12}}\right) \phi_{a}(2) d^{3} x_{2}\right\} \phi_{u}(1) .
\end{aligned}
$$


Como la variación de $\delta \phi_{i}^{*}$ es arbitraria, cada término en los paréntesis debe ser identicamente cero. Por lo tanto para cada orbital se debe de cumplir que

$$
\left.h_{1} \phi_{i}(1)+\sum_{j=1}^{N}\left\{J_{j}(1) \phi_{i}(1)-K_{j}(1) \phi_{i}(1)\right\}=\sum_{j=1}^{N} \epsilon_{j i} \phi_{j}(1)\right\} \text {. }
$$

Cuando la definición del operador de Fock se usa, la ec. (4.20) para los orbitales es

$$
f_{1} \phi_{i}(1)=\sum_{i=1}^{N} \epsilon_{j i} \phi_{j}(1)
$$

donde

$$
f_{1}=h_{1}+\sum_{u}\left\{J_{u}(1)-K_{u}(1)\right\}
$$

Con ésto, la energía total del sistema [ec. (4.11)] se reduce a

$$
\begin{aligned}
E & =\sum_{i=1}^{N}\left\langle\phi_{i}|h| \phi_{i}\right\rangle+\frac{1}{2} \sum_{i, j}^{N}\left\{\left\langle\phi_{i}\left|\hat{J}_{j}\right| \phi_{i}\right\rangle-\left\langle\phi_{i}\left|\hat{K}_{j}\right| \phi_{i}\right\rangle\right\} \\
& =\sum_{i=1}^{N}\left\langle\phi_{i}|\hat{f}| \phi_{i}\right\rangle-\frac{1}{2} \sum_{i, j}^{N}\left\{\left\langle\phi_{i}\left|\hat{J}_{j}-\hat{K}_{j}\right| \phi_{i}\right\rangle\right\} \\
& =\sum_{i=1}^{N} \epsilon_{i}-\frac{1}{2} \sum_{i, j}^{N}\left\{\left\langle\phi_{i}\left|\hat{J}_{j}-\hat{K}_{j}\right| \phi_{i}\right\rangle\right\}
\end{aligned}
$$

la cual nos dice que la energía del sistema no sólo es la suma de las energias de cada electrón, si no que existe una corrección debida al intercambio y repulsión de electrones.

La ec. (4.21) no es exactamente la forma estandard de las ecuaciones de HF, debido a que el conjunto de orbitales $\{\phi\}$ no es único. Es posible formar un nuevo conjunto de orbitales, cada uno siendo una combinación lineal de los orbitales $\phi$, sin cambiar la energía mínima $E$. En particular, es posible transformar el conjunto original en un nuevo conjunto ortonormal de orbitales canonicos, $\phi^{\prime}$, tal que el operador de Fock transformado $f_{1}^{\prime}$ es el mismo que $f_{1}$ y los elementos de matriz de los multiplicadores $\epsilon_{j i}$ se transforman en los elementos diagonales $\epsilon_{i}^{\prime}$. Los orbitales canónicos $\phi^{\prime}$ satisfacen la ecuación

$$
f_{1} \phi_{i}^{\prime}(1)=\epsilon_{i}^{\prime} \phi_{i}^{\prime}(1)
$$


que son las ec. de HF para los orbitales $\phi$.

Cada orbital debe ser obtenido mediante la solución de una ecuación de la forma (4.25), con el correspondiente operador de Fock $f_{1}$. Sin embargo, debido a que $f_{1}$ depende de los otros $N-1$ electrones (via el operador de intercambio), parecería que deberíamos de conocer las soluciones de antemano. Este es un dilema común en cálculos de estructura electrónica y es comunmente atacado mediante un procedimiento iterativo y deteniendose cuando las soluciones son autoconsistentes, de aquí el nombre de campo autoconsistente (self consistent field -SCF). En un procedimiento autoconsistente, un conjunto de orbitales prueba es utilizado para formular el operador de Fock, entonces las ecuaciones de HF [ec. (4.25)] se resuelven y se obtiene un nuevo conjunto de orbitales con los que se contruye de nuevo el operador de Fock y se repite el procedimiento hasta alcanzar algun criterio de convergencia.

El operador de Fock, definido en la ec. (4.21), depende de los $N$ orbitales ocupados. Sin embargo, una vez que los orbitales han sido determinados, el operador de Fock puede tratarse como un operador Hermitiano bién definido. En otras palabras, tendra un número infinito de soluciones $\phi_{u}$, cada uno teniendo energía $\epsilon_{u}$. En la práctica, por supuesto, tendremos que contentarnos con resolver la ec. (4.25) para un número finito $m$ de orbitales, con $m \geq N$.

Los $m$ orbitales obtenidos conforme el procedimiento Hartree-Fock SCF se arreglan en orden de incremento de energía, así, los $N$ orbitales de energía más baja se denominan, orbitales ocupados, u orbitales de agujero (hole). Los sobrantes $m-N$ orbitales se denominan orbitales virtuales o de partícula (particle).

\subsection{Aproximación de Hartree-Fock dependiente del tiempo: RPA}

Como los orbitales virtuales en el modelo HF SCF no representan realmente el espectro completo de excitación del sistema y como estamos interesados en obtener propiedades de transición de un estado inicial a un estado final, necesitamos expander el modelo HF SCF al caso en el que se considere la dependencia en el tiempo y así poder incluir de manera más realista las probabilidades de transición.

Una solución aproximada razonable a la ecuación dependiente del tiempo de 
Schrödinger para $N$ electrones parece ser el determinante

$$
\Phi(t)=\frac{1}{n !^{1 / 2}}\left|\phi_{1}(t) \phi_{2}(t) \cdots \phi_{N}(t)\right|
$$

en el cual cada espín-orbital varía con el tiempo de acuerdo a la ecuación de HartreeFock

$$
i \hbar \frac{\partial \phi_{m}(t)}{\partial t}=f_{1} \phi_{m}(t)
$$

El operador $f_{1}(t)$ incluye el tiempo de dos formas. Puede depender de un potencial $U(t)$ que dependa del tiempo y podría haber cambios en los campos Coulombiano y de intercambio conforme los electrones se moviesen. Esta solución no sería adecuada, debido a que en un estado estacionario, cada orbital tendría un factor dependiente del tiempo $e^{\left(-i \epsilon_{m} t / \hbar\right)}$ y $\Phi(t)$, [debido a la ec. (4.26)] tendría un factor $e^{-i\left(\epsilon_{1}+\epsilon_{2}+\cdots+\epsilon_{N}\right) t / \hbar}$, sin embargo, de la ecuación de Schrödinger

$$
\hat{H} \Phi(t)=i \hbar \frac{\partial \Phi(t)}{\partial t}
$$

la solución deberá tener un factor de la forma $e^{-i E t / \hbar} \operatorname{con} E$ la energía del sistema dada por la ec. (4.11), con lo que en la solución (4.26) faltaría en el factor exponencial el término para las energías de repulsión e intercambio electrónico.

Esta dificultad se corrige facilmente tomando la función de onda de la forma

$$
\Phi^{\prime}(t)=\Phi(t) e^{[i \gamma(t) / \hbar]},
$$

con el factor de fase

$$
\begin{aligned}
\gamma(t) & =\int_{0}^{t}\langle G(t)\rangle d t \\
& =-\frac{1}{2} \sum_{i, j}^{N}\left\{\left\langle\phi_{i}\left|\hat{J}_{j}-\hat{K}_{j}\right| \phi_{i}\right\rangle\right\}
\end{aligned}
$$

donde $G(t)$ es la energía de repulsión e intercambio electrónico, y mientras que los orbitales aún satisfacen la ec. (4.27), puesto que al sustituir en la ec. (4.28) se obtiene

$$
\begin{aligned}
\hat{H} \Phi & =\left[\sum_{i} \epsilon_{i}-\langle G\rangle\right] \Phi \\
& =E \Phi
\end{aligned}
$$


con lo que se ve que sí se satisface la ecuación de Schrödinger.

Para muchos propósitos, el factor de fase no es importante puesto que la parte dinámica en el comportamiento de los orbitales está gobernada por el operador de Fock [ec. (4.27)] y uno puede usar la función de onda no corregida $\Phi(t)$. Así, al tratamiento de Hartree-Fock dependiente del tiempo también se le conoce como aproximación de fase aleatoria (RPA) debido a la naturaleza aleatoria al modificarse ligeramente un orbital $\phi_{m}(t)$ para el factor de fase $\gamma(t)$.

Cuando una pequeña perturbación $F(t)$ actua sobre los electrones en su estado base $\Phi_{0}$, cada uno de los electrones modifica su estado ligeramente y la función de onda, a primer orden en $F$ puede expresarse como

$$
\Phi(t)=\Phi_{0}+\sum_{m, k} C_{m k}(t) \Phi(m \rightarrow k),
$$

donde $\Phi(m \rightarrow k)$ es el estado en el cual un electrón saltó desde $\phi_{m}$ a $\phi_{k}$. Así, las ecuaciones de movimiento de Hartree-Fock (4.27) nos dan

$$
\begin{aligned}
i \hbar \frac{d C_{m k}}{d t} & =\left(\epsilon_{k}-\epsilon_{m}\right) C_{m k}+\sum_{j n}\left\{\langle k n \| m j\rangle C_{n j}+\langle k j \| m n\rangle C_{n j}^{*}\right\}-F_{k m}(t) \\
-i \hbar \frac{d C_{m k}^{*}}{d t} & =\left(\epsilon_{k}-\epsilon_{m}\right) C_{m k}^{*}+\sum_{j n}\left\{\langle m n \| k j\rangle C_{n j}+\langle m j \| k n\rangle C_{n j}^{*}\right\}-F_{m k}(t)
\end{aligned}
$$

para los coeficientes, y donde $\langle a b|| c d\rangle=\langle a b \mid c d\rangle-\langle a b \mid d c\rangle$. Si la fuerza es de tipo oscilatorio con un factor $e^{-i \omega t}$, los coeficientes $C$ contendrán términos con factores de la forma $e^{ \pm i \omega t}$

$$
\begin{aligned}
& C_{m k}=X_{m k} e^{-i \omega t}+Y_{m k}^{*} e^{+i \omega t} \\
& C_{m k}^{*}=X_{m k}^{*} e^{+i \omega t}+Y_{m k} e^{-i \omega t}
\end{aligned}
$$

debiéndose al hecho de que ambos $C$ y $C^{*}$ aparecen en la ecuación de movimiento para $C$. Sustituyendo la ecuación anterior en (4.34) nos conduce a las ecuaciones para los coeficientes $X$ y $Y$

$$
\begin{aligned}
\hbar \omega X_{m k} & =\left(\epsilon_{k}-\epsilon_{m}\right) X_{m k}+\sum_{j n}\left\{\langle k n \| m j\rangle X_{n j}+\langle k j \| m n\rangle Y_{n j}^{*}\right\}-F_{k m}(t) \\
-\hbar \omega Y_{m k} & =\left(\epsilon_{k}-\epsilon_{m}\right) Y_{m k}^{*}+\sum_{j n}\left\{\langle m n \| k j\rangle X_{n j}+\langle m j \| k n\rangle Y_{n j}^{*}\right\}-F_{m k}(t)
\end{aligned}
$$


Las soluciones de estas ecuaciones corresponden a oscilaciones forzadas, pero cuando $F=0$, pueden ocurrir oscilaciones libres a ciertas frecuencias $\omega_{a}$. Físicamente, ésto significa que para una transición del sistema en el estado $k$ a un estado $m$, la energía de transición sería $\epsilon_{m}-\epsilon_{k}$, pero debido a que el sistema tiene N-electrones acoplados durante la transición, el cambio del orbital $\phi_{k}$ a $\phi_{m}$ produce un reajuste de los electrones restantes, con lo cual la energía de la transición se corrige por un factor que depende de dichas distribuciones electrónicas. Esto es lo que determina los niveles de excitación naturales. En el caso de la presencia de una perturbación, estos se ven afectados por dicha perturbación, como se ve de la ecuación anterior. Estas son las ecuaciones que gobiernan las funciones de onda de Hartree-Fock en el modelo RPA.

Con esto estamos en posición de demostrar si la regla de suma de Bethe se satisface en esta aproximación.

\subsection{La regla de suma de Bethe en RPA}

La regla de suma TRK sólo se aplica cuando no hay momento transferido durante la colisión entre el proyectil y blanco, y así, no es un buén criterio para determinar una buena base en problemas que incluyen transferencia de momento. Su generalización es la regla de suma de Bethe (BSR), i.e. ec. (3.26) la cual es válida para todas las transferencias de momento cinemáticamente permisibles, y así puede servir como un buén criterio para determinar conjuntos de base cuando uno se encuentre ante problemas que consideren distribuciones de GOS y por lo tanto, permitirnos calcular el poder de frenamiento por ejemplo. Así, examinemos primero los GOS en la aproximación RPA y mostremos que la BSR se satisface además en esta aproximación para una base completa.

En la aproximación de fase aleatoria, las amplitudes de transición para un operador de un electrón puede escribirse como

$$
\langle 0|\mathbf{O}| l\rangle=\sum_{h, p}\left[\langle p|\mathbf{O}| h\rangle^{*} X_{h, p}^{l}+\langle h|\mathbf{O}| p\rangle^{*} Y_{h, p}^{l}\right]
$$

donde $|p\rangle$ y $|h\rangle$ son el conjunto completo de solucion es de partículas y hoyos, respectivamente, para las ecuaciones ordinarias de Hartree-Fock 


$$
\left[h_{o}+J-K\right]|i\rangle=\epsilon_{i}|i\rangle .
$$

Aqui $h_{0}$ es el operador de energía cinética y atracción nuclear, mientras que $J$ y $K$ son los operadores de Coulomb e intercambio respectivamente. Los coeficientes $X$ y $Y$ son soluciones a las ecuaciones RPA

$$
\begin{aligned}
\epsilon_{l} X_{h, p}^{l} & =\left(\epsilon_{p}-\epsilon_{h}\right) X_{h, p}^{l}+\sum_{h^{\prime}, p^{\prime}}\left\{\left\langle p h^{\prime} \| h p^{\prime}\right\rangle X_{h^{\prime}, p^{\prime}}^{l}+\left\langle p p^{\prime} \| h h^{\prime}\right\rangle Y_{h^{\prime} p^{\prime}}^{l}\right\} \\
-\epsilon_{l} Y_{h, p}^{l} & =\left(\epsilon_{p}-\epsilon_{h}\right) Y_{h, p}^{l}+\sum_{h^{\prime}, p^{\prime}}\left\{\left\langle h p^{\prime} \| p h^{\prime}\right\rangle Y_{h^{\prime}, p^{\prime}}^{l}+\left\langle h h^{\prime} \| p p^{\prime}\right\rangle X_{h^{\prime} p^{\prime}}^{l}\right\}
\end{aligned}
$$

los cuales son las soluciones para la l-ésima transición con energía $\epsilon_{l}$. Combinando las ecuaciones (4.36) y (4.38), encontramos una expresión para el elemento de matriz $\epsilon_{l}\langle 0|\mathbf{M}| l\rangle$ dado por

$$
\begin{aligned}
& \epsilon_{l}\langle 0|\mathbf{O}| l\rangle=\sum_{h, p}\left\{\quad \left[\left(\epsilon_{p}-\epsilon_{h}\right)\langle p|\mathbf{O}| h\rangle^{*}-\langle p|\left[\mathbf{O}, K|h\rangle^{*}\right] X_{h, p}^{l}-\right.\right. \\
& \left.\left[\left(\epsilon_{p}-w_{h}\right)\langle h|\mathbf{O}| p\rangle^{*}+\langle h|[\mathbf{O}, K]| p\rangle^{*}\right] Y_{h, p}^{*}\right\} .
\end{aligned}
$$

El término que aparece dentro del conmutador aparece como consecuencia de que un operador de un electrón no conmuta con el operador de intercambio.

De las ecuaciones (4.37), y (4.36) se puede demostrar que

$$
\left(\epsilon_{p}-\epsilon_{h}\right)\langle h|\mathbf{O}| p\rangle=\left\langle h\left|\left[\mathbf{O}, h_{0}\right]\right| p\right\rangle-\langle h|[\mathbf{O}, K]| p\rangle
$$

Usando ésto en la ec. (4.39) obtenemos que la dependencia del operador de intercambio se puede eliminar obteniendose que

$$
\epsilon_{l}\langle 0|\mathbf{O}| l\rangle=\sum_{h, p}\left[\left\langle p\left|\left[h_{0}, \mathbf{O}\right]\right| h\right\rangle^{*} X_{h, p}^{l}+\left\langle h\left|\left[h_{0}, \mathbf{O}\right]\right| p\right\rangle^{*} Y_{h, p}^{l}\right] .
$$

Es útil ahora adoptar una notación de doble vector, tal que $\left|\mathbf{X}^{l}, \mathbf{Y}^{l}\right\rangle$ representa el conjunto de coeficientes $X_{h, p}^{l}, Y_{h, p}^{l}$. En esta notación el resultado anterior se puede escribir como

$$
\epsilon_{l}\langle 0|\mathbf{O}| l\rangle=\left\langle\mathbf{B}^{*}, \tilde{\mathbf{B}}^{*} \mid \mathbf{X}^{l}, \mathbf{Y}^{l}\right\rangle
$$

donde

$$
\mathbf{B}=\left[h_{0}, \mathbf{O}\right]
$$


Uno obtiene así una regla de suma para los elementos de matriz pesados por la energía como

$$
2 \sum_{l>0} \epsilon_{l}\left|O_{0, l}\right|^{2}=\sum_{l}\left\langle\mathbf{B}^{*}, \tilde{\mathbf{B}}^{*} \mid \mathbf{X}^{l}, \mathbf{Y}^{l}\right\rangle\left\langle\mathbf{X}^{l},-\mathbf{Y}^{l} \mid \mathbf{O},-\tilde{\mathbf{O}}\right\rangle
$$

Usando la relación de completez

$$
\sum_{ \pm l}\left|\mathbf{X}^{l}, \mathbf{Y}^{l}\right\rangle\left\langle\mathbf{X}^{l},-\mathbf{Y}^{l}\right|=1
$$

encontramos que la regla de suma, para un operador de un electrón se puede escribir como

$$
\begin{aligned}
\sum_{l>0} F_{0 l} & =\left\langle\mathbf{B}^{*}, \tilde{\mathbf{B}}^{*} \mid \mathbf{O},-\tilde{\mathbf{O}}\right\rangle \\
& =\sum_{h, p}\left[\left\langle p\left|\left[h_{0}, \mathbf{O}\right]\right| h\right\rangle^{*}\langle p|\mathbf{O}| h\rangle-\left\langle h\left|\left[h_{0}, \mathbf{O}\right]\right| p\right\rangle^{*}\langle h|\mathbf{O}| p\rangle\right]
\end{aligned}
$$

Esta ecuación es la forma general de la regla de suma para un operador de un electrón $\mathbf{O}$ expresado en términos de el cuadrado de las transiciones de momento y sus energías de excitación dentro de la aproximación RPA.

Para continuar con la demostración de la BSR apliquemos la ec. (4.46) al caso donde el operador toma los casos especiales:

- Primero consideremos el operador dipolar $(\mathbf{O}=\mathbf{r})$. El elemento de matriz de el lado derecho de la ec. (4.46) es justamente las transiciones de momento dipolar, y el conmutador $\mathbf{B}$ es entonces $-i \mathbf{p}$. Como estamos considerando las soluciones exactas a la aproximación RPA, existe un conjunto completo de estados partícula-hoyo que satisfacen la relación de completez

$$
\sum_{h}|h\rangle\left\langle h\left|+\sum_{p}\right| p\right\rangle\langle p|=1
$$

y así

$$
\sum_{l>0} f_{0 l}=i \sum_{h}\langle h|[\mathbf{p}, \mathbf{r}]| h\rangle
$$

puesto que $[\mathbf{p}, \mathbf{r}]=-3 i$ la regla de suma de TRK se recupera inmediatamente. 
- Ahora consideremos el caso en el que $\mathbf{O}=e^{-i \mathbf{q} \cdot \mathbf{r}}$, i.e. un operador de onda plana, o el operador que aparece en la BSR. Usando un procedimiento similar al utilizado en el ejemplo anterior y notando que

$$
\left[\mathbf{p}, e^{-i \mathbf{q} \cdot \mathbf{r}}\right]=-\mathbf{q} e^{-i \mathbf{q} \cdot \mathbf{r}}
$$

el conmutador en la ec. (4.46) es

$$
\mathbf{B}=e^{-i \mathbf{q} \cdot \mathbf{r}}\left(-\frac{q^{2}}{2}+\mathbf{q} \cdot \mathbf{p}\right)
$$

con lo cual al usar el hecho de que los estados particula-hoyo son completos, encontramos que la ec. (4.46) se reduce al número de electrones $N$, i.e. la regla de suma BSR se satisface.

Así, la regla de suma BSR se satisface exactamente en la aproximación RPA, si una base completa es usada, para todas las transferencias de momento. Por lo tanto, como en el caso de la regla de suma TRK, cuando se consideran propiedades de transición opticas $(q=0)$, esperamos que la regla de suma BSR sea útil al evaluar conjuntos de bases cuando se calculen las distribuciones de los GOS, por ejemplo, en el uso del poder de frenamiento. Por otro lado, debemos notar que la calidad del conjunto base es dependiente de $\mathbf{q}$ [97] y que por lo tanto, debemos tener cuidado al evaluar la BSR para varios valores de $\mathbf{q}$. Esta parte se encuentra actualemnte en proceso de investigación. 


\section{Capítulo 5}

\section{Regla de suma de Bethe con un campo electromagnético externo}

\section{$5.1 \quad$ Resumen}

En éste capítulo, analizamos la regla de suma de Bethe (BSR) para un sistema electrónico inmerso en un campo electromagnético para un Hamiltoniano no relativista. Esto es con el fín de ver, desde primeros principios, si la BSR se satisface, y por lo tanto poder formular un criterio para la determinación de la completez de bases utilizadas en este tipo de problemas.

\subsection{Introducción}

Como hemos visto ya en los capítulos anteriores, las Tensiones de Oscilador Generalizado (GOS) son de gran importancia en la descripción de la estructura electrónica de átomos y moléculas, en particular son usadas como indicadores de la calidad de los conjuntos de bases para el cálculo de propiedades atómicas y moleculares [97].

Recientemente el estudio de átomos y moléculas en ambientes extremos se ha incrementado en física y química [103, 104, 105], por ejemplo, con el mejoramiento de las capacidades magnéticas de varios laboratorios especializados con flujos de campos magnéticos de hasta varios cientos de Teslas y con campos astrofísicos de varios ordenes de magnitud mayores, en donde la aceleración de protones en ráfagas solares o vientos interestelares colisionan con átomos y moléculas, la pregunta sobre el entendimiento y predicción de propiedades de átomos y moleculas en campos 
intensos es de vital interés tanto teórica como computacionalmente.

Hemos visto en los capítulos anteriores que la energía e ímpetu transferido a un blanco átomico o molecular con $N$ electrones por un proyectil que incide sobre éstos está descrita completamente por las GOS [73]

$$
F_{n 0}(\mathbf{q})=\frac{2 m E_{n 0}}{\hbar^{2} q^{2}}\left|\left\langle n\left|\sum_{j=1}^{N} e^{-i \mathbf{q} \cdot \mathbf{r}_{j}}\right| 0\right\rangle\right|^{2}
$$

donde el blanco es excitado desde el estado inicial $|0\rangle$ a el estado final $|n\rangle ; E_{n 0}=$ $E_{n}-E_{0}$ es la energía transferida y $\hbar \mathbf{q}$ es el ímpetu transferido durante la colisión. La suma es sobre todos los electrones del blanco con las coordenadas $\mathbf{r}_{j}$. Cuando se suma sobre todos los estados excitados discretos y contínuos, obtenemos la Regla de suma de Bethe

$$
\sum_{n>0} F_{n 0}(\mathbf{q})=N
$$

lo cual requiere que los GOS para el sistema sumados nos dén el número de electrones del sistema para todos los valores del ímpetu transferido q.

Cuando no hay ímpetu transferido, por ejemplo para transiciones dipolares ópticas, el límite para $q \rightarrow 0$ de la Regla de suma de Bethe, conocida como la Regla de Thomas-Reiche-Kuhn (TRK) [95, 96] es

$$
\sum_{n>0} f_{n 0}=N
$$

Aquí, las $\left\{f_{n 0}\right\}$ son las tensiones de oscilador dipolar. Físicamente la regla de suma nos dice que, para una base completa la suma de las tensiones de oscilador dan el número de electrones, o en otras palabras, que la energía transferida al sistema sumada sobre todos los modos internos de excitación debe ser la misma que la energía transferida a $\mathrm{N}$ electrones libres [73].

Para determinar si la Regla de Bethe se satisface cuando un átomo o molécula en cuestión esta sujeta a un campo electromagnético estático externo, necesitamos determinar el Hamiltoniano para dicho sistema. 


\subsection{Hamiltoniano para un campo electromagné- tico}

Puesto que los efectos relativistas no son importantes para campos por debajo de los $10^{8}$ teslas [105] nuestro tratamiento se basará en un Hamiltoniano no-relativista. Siguiendo el procedimiento estandard [102], consideremos la fuerza de Lorentz actuando sobre una partícula cargada bajo la acción de un campo electromagnético. En este caso la fuerza puede ser escrita como

$$
\mathbf{F}=q\left[\mathbf{E}+\frac{1}{c}(\mathbf{v} \times \mathbf{B})\right]
$$

Expresando los campos eléctrico, E, y magnético, B, en términos del potencial escalar $\phi$ y el potencial vectorial $\mathbf{A}$,

$$
\begin{aligned}
& \mathbf{E}=-\nabla \phi-\frac{1}{c} \frac{\partial \mathbf{A}}{\partial t}, \\
& \mathbf{B}=\nabla \times \mathbf{A} .
\end{aligned}
$$

Estas ecuaciones no especifican a $\phi$ y $\mathbf{A}$ univocamente. Las ecuaciones de Maxwell toman su forma más simple cuando el potencial escalar $\phi$ y el potencial vectorial A se relacionan uno a otro a través de la condición de Lorentz

$$
\nabla \cdot \mathbf{A}+\frac{1}{c} \frac{\partial \phi}{\partial t}=0
$$

Sustituyendo la ecuación (5.5) en la ec. (5.4) obtenemos que

$$
\mathbf{F}=q\left\{-\nabla \phi-\frac{1}{c}\left[\frac{\partial \mathbf{A}}{\partial t}-\mathbf{v} \times(\nabla \times \mathbf{A})\right]\right\} .
$$

Por otro lado, puesto que

$$
\frac{d \mathbf{A}}{d t}=\frac{\partial \mathbf{A}}{\partial t}+(\mathbf{v} \cdot \nabla) \mathbf{A}
$$

y

$$
\mathbf{v} \times(\nabla \times \mathbf{A})=\nabla(\mathbf{v} \cdot \mathbf{A})-(\mathbf{v} \cdot \nabla) \mathbf{A}
$$

la ec. (5.7) puede re-escribirse como

$$
\mathbf{F}=q\left[-\nabla\left(\phi-\frac{\mathbf{v} \cdot \mathbf{A}}{c}\right)-\frac{1}{c} \frac{d \mathbf{A}}{d t}\right]
$$


De esta ecuación se puede ver que escogiendo el potencial generalizado $U$ como

$$
U=q\left(\phi-\frac{\mathbf{v} \cdot \mathbf{A}}{c}\right)
$$

(puesto que $\phi$ y A no dependen de la velocidad de la partícula), el lagrangiano del sistema es

$$
L=\frac{m \dot{r}^{2}}{2}-q \phi+\frac{q}{c} \mathbf{v} \cdot \mathbf{A} .
$$

Ahora, ya que tenemos el Lagrangiano que describe la interacción de la partícula cargada con un campo electromagnético externo, necesitamos encontrar el Hamiltoniano, para esto necesitamos el ímpetu generalizado, i.e.

$$
p_{i}=\frac{\partial L}{\partial v_{i}}=m v_{i}+\frac{q}{c} A_{i}
$$

donde $i=x, y, z$ o vectorialmente

$$
\mathbf{p}=m \dot{\mathbf{r}}+\frac{q}{c} \mathbf{A}
$$

con lo que ahora nos resta formar el Hamiltoniano, el cual está definido por

$$
\begin{aligned}
H & =\mathbf{p} \cdot \dot{\mathbf{r}}-L \\
& =\frac{1}{m} \mathbf{p} \cdot\left(\mathbf{p}-\frac{q}{c} \mathbf{A}\right)-\frac{1}{2 m}\left(\mathbf{p}-\frac{q}{c} \mathbf{A}\right)^{2}+q \phi-\frac{q}{m}\left(\mathbf{p}-\frac{q}{c} \mathbf{A}\right) \cdot \frac{\mathbf{A}}{c} \\
& =\frac{\left(\mathbf{p}-\frac{q}{c} \mathbf{A}\right)^{2}}{2 m}+q \phi .
\end{aligned}
$$

Pero como estamos considerando interacción entre los campos electromagnéticos y los electrones de la molécula o átomo, entonces la interacción sera con los electrones que forman la estructura electrónica $(q=-e)$ y además los electrones están bajo la influencia de un potencial electrónico de repulsión entre ellos y la atracción del núcleo. Así, designando a éste por $V$ tenemos que el Hamiltoniano que describirá nuestra interacción es

$$
H=\sum_{i=1}^{N_{2}} \frac{\left(\mathbf{p}_{i}+\frac{e}{c} \mathbf{A}_{i}\right)^{2}}{2 m}+V-e \phi .
$$

La ecuación de Schrödinger para el sistema en presencia de campos electromagnéticos es entonces

$$
H|n\rangle=E_{n}|n\rangle
$$


donde $|n\rangle$ es el conjunto completo de soluciones que satisfacen la relación de completez

$$
\sum_{n}|n\rangle\langle n|=1 .
$$

Con esto ya estamos en posición de calcular la regla de suma de Bethe para el sistema en presencia de campos electromagnéticos externos estáticos.

\subsection{Regla de suma de Bethe}

Siguiendo la deducción estandard [72] sustituimos la ec. (5.17) dentro de la definición de las tensiones de Oscilador Generalizado [ec. (5.1)] y haciendo uso de la relación de completez encontramos que la regla de suma de Bethe se puede escribir como

$$
\sum_{n>0} F_{0 n}(\mathbf{q})=\frac{2 m}{\hbar^{2} q^{2}}\left\langle 0\left|\sum_{j} e^{-i \mathbf{q} \cdot \mathbf{r}_{j}}\left[H, \sum_{k} e^{i \mathbf{q} \cdot \mathbf{r}_{k}}\right]\right| 0\right\rangle .
$$

De esta expresión, sólo nos resta evaluar el conmutador. Como ni el potencial electrónico $V$ ni el potencial escalar eléctrico $\phi$ son funciones de la velocidad o ímpetu, uno obtiene que

$$
\begin{aligned}
{\left[H, \sum_{k} e^{i \mathbf{q} \cdot \mathbf{r}_{k}}\right] } & =\sum_{k} \frac{1}{2 m}\left[\left(\mathbf{p}_{k}+\frac{e}{c} \mathbf{A}_{k}\right)^{2}, e^{i \mathbf{q} \cdot \mathbf{r}_{k}}\right] \\
& =\sum_{k} \frac{e^{i \mathbf{q} \cdot \mathbf{r}_{k}}}{2 m}\left\{\hbar^{2} q^{2}+2 \hbar \mathbf{q} \cdot\left(\mathbf{p}_{k}+\frac{e}{c} \mathbf{A}_{\mathbf{k}}\right)\right\},
\end{aligned}
$$

por lo que la regla de suma de Bethe es

$$
\begin{aligned}
\sum_{n>0} F_{0} n(\mathbf{q})= & N_{2}+\frac{2}{\hbar q^{2}} \mathbf{q} \cdot\left\langle 0\left|\sum_{l}\left(\mathbf{p}_{l}+\frac{e}{c} \mathbf{A}_{l}\right)\right| 0\right\rangle+ \\
& \frac{1}{\hbar^{2} q^{2}} \sum_{j \neq k}\left\langle 0\left|e^{i \mathbf{q} \cdot\left(\mathbf{r}_{k}-\mathbf{r}_{j}\right)}\left\{\hbar^{2} q^{2}+2 \hbar \mathbf{q} \cdot\left(\mathbf{p}_{k}+\frac{e}{c} \mathbf{A}_{k}\right)\right\}\right| 0\right\rangle,
\end{aligned}
$$

donde los dos primeros términos surgen del caso cuando $j=k$. Por otra parte se puede demostrar fácilmente que

$$
\begin{aligned}
{\left[H, \mathbf{r}_{l}\right] } & =\frac{1}{2 m} \sum_{k}\left[\left(\mathbf{p}_{\mathbf{k}}+\frac{e}{c} \mathbf{A}_{k}\right)^{2}, \mathbf{r}_{l}\right] \\
& =-\frac{3 i \hbar}{m}\left(\mathbf{p}_{\mathbf{1}}+\frac{e}{c} \mathbf{A}_{l}\right) .
\end{aligned}
$$


Así, el segundo término en la ec. (5.21) es proporcional a $\left\langle 0\left|\sum_{l}\left[H, \mathbf{r}_{l}\right]\right| 0\right\rangle$, el cual es claramente cero, debido a que $|0\rangle$ es la solución del estado base de la ecuación de Schrödinger, con lo que la ecuación (5.21) se reduce a

$$
\sum_{n>0} F_{0 n}(\mathbf{q})=N_{2}+\frac{1}{\hbar^{2} q^{2}} \sum_{j \neq k}\left\langle 0\left|e^{i \mathbf{q} \cdot\left(\mathbf{r}_{k}-\mathbf{r}_{j}\right)}\left\{\hbar^{2} q^{2}+2 \hbar \mathbf{q} \cdot\left(\mathbf{p}_{k}+\frac{e}{c} \mathbf{A}_{k}\right)\right\}\right| 0\right\rangle .
$$

Si escribimos la función de onda explícitamente como $\psi_{0}(\mathbf{r})$ y usamos el hecho de que $\mathbf{p}_{k}=-i \hbar \nabla_{k}$, entonces el segundo término de la ec. (5.23) se puede escribir como

$$
\begin{gathered}
\sum_{j \neq k}\left\langle 0\left|e^{i \mathbf{q} \cdot\left(\mathbf{r}_{k}-\mathbf{r}_{j}\right)}\left\{\hbar^{2} q^{2}+2 \hbar \mathbf{q} \cdot\left(\mathbf{p}_{k}+\frac{e}{c} \mathbf{A}_{k}\right)\right\}\right| 0\right\rangle= \\
i \hbar \mathbf{q} \cdot \sum_{j \neq k} \int d \tau e^{i \mathbf{q} \cdot\left(\mathbf{r}_{k}-\mathbf{r}_{j}\right)}\left[\left(\nabla_{k} \psi_{0}^{*}(\mathbf{r})\right) \psi_{0}(\mathbf{r})-\psi_{0}^{*}(\mathbf{r}) \nabla_{k} \psi_{0}(\mathbf{r})+\frac{2 e \mathbf{A}_{k} \psi_{0}^{*}(\mathbf{r}) \psi_{0}(\mathbf{r})}{i \hbar c}\right] .
\end{gathered}
$$

Para el Hamiltoniano del tipo que estamos considerando aquí [ec. (5.16)], la densidad de corriente para el k-ésimo electrón está dada por [106]

$$
\mathbf{J}_{k}^{0}=\frac{e \hbar}{2 i m}\left[-\left(\nabla_{k} \psi_{0}^{*}(\mathbf{r})\right) \psi_{0}(\mathbf{r})+\psi_{0}^{*}(\mathbf{r}) \nabla_{k} \psi_{0}(\mathbf{r})-\frac{2 e \mathbf{A}_{k} \psi_{0}^{*}(\mathbf{r}) \psi_{0}(\mathbf{r})}{i \hbar c}\right]
$$

el cuál es precisamente el término entre paréntesis y así, encontramos que

$$
\sum_{j \neq k}\left\langle 0\left|e^{i \mathbf{q} \cdot\left(\mathbf{r}_{k}-\mathbf{r}_{j}\right)}\left\{\hbar^{2} q^{2}+2 \hbar \mathbf{q} \cdot\left(\mathbf{p}_{k}+\frac{e}{c} \mathbf{A}_{k}\right)\right\}\right| 0\right\rangle=\frac{2 m}{e} \mathbf{q} \cdot \sum_{j \neq k} \int d \tau e^{i \mathbf{q} \cdot\left(\mathbf{r}_{k}-\mathbf{r}_{j}\right)} \mathbf{J}_{k}^{0} .
$$

El índice superior indica que nos estamos refiriendo al estado base. Por otro lado integrando por partes $\mathbf{J}_{k}^{0}$ encontramos que

$$
\int d \tau \mathbf{J}_{k}^{0}=\frac{e}{m}\left\langle 0\left|\mathbf{p}_{k}+\frac{e}{c} \mathbf{A}_{k}\right| 0\right\rangle
$$

Del argumento usado en conexión con el desarrollo de la ecuación (5.23), vemos que el término de la derecha de la ecuación (5.27) es cero, y como $\mathbf{J}_{k}^{0}$ es un vector, ésto implica que $\mathbf{J}_{k}^{0}=0$, con lo que la ecuación (5.23) se reduce a la ec. (5.2) que es la regla de suma de Bethe.

Esto demuestra que la regla de suma de Bethe y por lo tanto la regla de suma de Thomas-Reiche-Kuhn se satisfacen en el caso de tener las soluciones completas, para un campo electromagnético externo. Notemos que no hemos hecho ninguna 
suposición en este desarrollo, acerca de que las funciones de onda deban ser reales, por lo que estas reglas de suma se aplican a estados con funciones de onda complejas, así como a estados degenerados. Este resultado parece razonable, puesto que hemos partido de un Hamiltoniano no-relativista [ec. (5.16)], en el cual no se contempla creación ó aniquilación de partículas.

\subsection{Análisis perturbativo}

El desarrollo anterior reside principalmente en la suposición de la completez de las funciones de onda para el Hamiltoniano. Si deseamos usar la regla de suma de Bethe y la regla de suma de Thomas-Reiche-Kuhn como una medida de la precisión de la base mediante la comparación de éstas con el número de electrones en el sistema nosotros debemos enfrentarnos con funciones de onda aproximadas. Puesto que la regla de suma de Bethe exacta en presencia de un campo electromagnético (superíndice B) es igual a la regla de suma calculada sin campo electromagnético, i.e. cuando $B=0$ (superíndice (0)), y a su vez, igual al número de electrones, con lo que si expandemos la regla de suma de Bethe en términos del campo electromagnético, concluimos que las correcciones a orden superior en el campo aplicado deben ser cero y así

$$
\sum_{n} F_{0 n}^{B}(\mathbf{q})=\sum_{n} F_{0 n}^{(0)}(\mathbf{q})=N_{2}
$$

para todo $\mathbf{q}$.

Así, las desviaciones de las correcciones orden por orden nos pueden dar una visión de la completez de una base utilizada computacionalmente. Como un ejemplo, analicemos el caso de la corrección a primer orden.

Consideremos una expansión perturbativa de las Tensiones de Oscilador Generalizado y de ahí, la regla de suma de Bethe resultante. Aunque estamos interesados en campos intensos disponibles en los laboratorios, éstos son aún pequeños comparados con la unidad atómica de campo magnetico ${ }^{\text {i }}$, así la teoría de perturbaciones se puede aplicar sin restricciones. El resto del tratamiento se restringirá al caso de estados base no-degenerados y usaremos teoría de perturbaciones estandard de Rayleigh-Schrödinger.

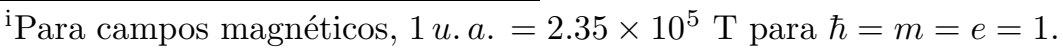


Consideremos a nuestro sistema en presencia de un campo magnético estático externo y consideremos a éste como una perturbación. En este caso la norma de Lorentz se reduce a

$$
\nabla \cdot \mathbf{A}=0
$$

que es la norma de Coulomb y tal que

$$
\mathbf{E}=-\nabla \phi(\mathbf{r}) \quad \text { para } \quad \mathbf{A} \neq \mathbf{A}(t)
$$

Como el campo magnético es constante, escogemos a el potencial vectorial magnético como

$$
\mathbf{A}_{l}=\frac{1}{2} \mathbf{B} \times \mathbf{r}_{l}
$$

el cual satisface la norma de Coulomb, ec. (5.29). Aquí, $\left\{\mathbf{r}_{l}\right\}$ son las coordenadas electrónicas relativas a un orígen de norma arbitrario.

Si ahora hacemos una expansión perturbativa de las Tensiones de Oscilador Generalizado en presencia del campo magnético estático aplicado, entonces obtenemos

$$
F_{0 n}^{B}(\mathbf{q})=F_{0 n}^{(0)}(\mathbf{q})+B F_{0 n}^{(1)}(\mathbf{q})+B^{2} F_{0 n}^{(2)}(\mathbf{q})+\cdots,
$$

donde el superíndice identifica las Tensiones de Oscilador Generalizado en presencia de el campo y el número en el paréntesis indica el orden de la perturbación. En este caso, cuando expandemos el Hamiltoniano encontramos que

$$
\sum_{l} \mathbf{A}_{l} \cdot \mathbf{p}_{l}=\frac{1}{2} \mathbf{B} \cdot \mathbf{L}
$$

donde el operador de momento angular total $\mathbf{L}=\sum_{l} \mathbf{L}_{l}$ es la suma del operador de momento angular de cada electrón, tal que el Hamiltoniano del sistema esta dado por

$$
\begin{aligned}
H= & {\left[\sum_{l} \frac{p_{l}^{2}}{2 m}+V(\mathbf{r})-e \phi(\mathbf{r})\right]+\left[\frac{e}{2 m} \sum_{l} \mathbf{B} \cdot \mathbf{L}_{l}\right]+} \\
& {\left[\frac{e^{2}}{8 m} \sum_{l} \mathbf{B} \cdot\left(r_{l}^{2}-\mathbf{r}_{l} \mathbf{r}_{l}\right) \cdot \mathbf{B}\right] } \\
= & H^{(0)}+H^{(1)}+H^{(2)}
\end{aligned}
$$

Usando teoría de perturbaciones a primer orden en la función de onda, lo que nos garantiza una corrección a la energía a segundo orden i.e.

$$
\psi_{n}=\psi_{n}^{(0)}+\psi_{n}^{(1)} \quad \text { y } \quad E_{n}=E_{n}^{(0)}+E_{n}^{(1)}+E_{n}^{(2)},
$$


donde

$$
\begin{aligned}
\psi_{n}^{(1)} & =\sum_{k} a_{k n} \psi_{k}^{(0)}, \\
a_{k n} & =\frac{H_{k n}^{(1)}}{E_{n}^{(0)}-E_{k}^{(0)}}, \\
E_{n}^{(1)} & =H_{n n}^{(1)}, \\
E_{n}^{(2)} & =H_{n n}^{(2)}+\sum_{k} \frac{H_{n k}^{(1)} H_{k n}^{(1)}}{E_{n}^{(0)}-E_{k}^{(0)}},
\end{aligned}
$$

con lo que uno puede escribir para cualquier operador de un electrón $O$ a primer orden

$$
\left|\left\langle\psi_{0}|O| \psi_{n}\right\rangle\right|^{2}=O_{0 n}^{(0)}+O_{0 n}^{(1)}
$$

y

$$
\begin{aligned}
O_{0 n}^{(1)}= & \left\langle\psi_{0}^{(0)}\left|O^{*}\right| \psi_{n}^{(0)}\right\rangle\left\langle\psi_{n}^{(1)}|O| \psi_{0}^{(0)}\right\rangle+\left\langle\psi_{0}^{(0)}\left|O^{*}\right| \psi_{n}^{(1)}\right\rangle\left\langle\psi_{n}^{(0)}|O| \psi_{0}^{(0)}\right\rangle \\
& +\left\langle\psi_{0}^{(0)}\left|O^{*}\right| \psi_{n}^{(0)}\right\rangle\left\langle\psi_{n}^{(0)}|O| \psi_{0}^{(1)}\right\rangle+\left\langle\psi_{0}^{(1)}\left|O^{*}\right| \psi_{n}^{(0)}\right\rangle\left\langle\psi_{n}^{(0)}|O| \psi_{0}^{(0)}\right\rangle .
\end{aligned}
$$

Con lo que el análogo para las Tensiones de Oscilador Generalizado es entonces

$$
\begin{aligned}
G_{0 n} & =E_{n 0}\left|\left\langle\psi_{0}|O| \psi_{n}\right\rangle\right|^{2} \\
& =G_{o n}^{(0)}+G_{0 n}^{(1)} .
\end{aligned}
$$

Aqui, el término $G_{o n}^{(0)}$ se define como

$$
G_{o n}^{(0)}=E_{n 0}^{(0)} O_{0 n}^{(0)}
$$

y

$$
G_{0 n}^{(1)}=E_{n 0}^{(0)} O_{0 n}^{(1)}+E_{n 0}^{(1)} O_{0 n}^{(0)} .
$$

Podemos obtener una expresión más explícita si utilizamos la definición de las energías perturbadas en términos de los elementos de matriz de la perturbación, ec. (5.37) y la ec. (5.33), con lo que la expresión ya simplificada nos da

$$
G_{0 n}=E_{n 0}^{(0)} O_{0 n}^{(0)}+
$$




$$
\begin{aligned}
& \frac{e}{2 m} \mathbf{B} \cdot \sum_{k}\left\{\frac{O_{0 n}^{*} \mathbf{L}_{n k} O_{k 0}}{E_{n k}^{(0)}}+\frac{O_{0 k}^{*} \mathbf{L}_{k n} O_{n 0}}{E_{n k}^{(0)}}+\right. \\
& \left.\frac{O_{0 n}^{*} O_{n k} \mathbf{L}_{k 0}}{E_{0 k}^{(0)}}+\frac{\mathbf{L}_{0 k} O_{k n}^{*} O_{n 0}}{E_{0 k}^{(0)}}\right\} E_{n 0}^{(0)},
\end{aligned}
$$

donde $\mathbf{L}_{n k}=\left\langle\psi_{n}^{(0)}|\mathbf{L}| \psi_{k}^{(0)}\right\rangle$ y $O_{k n}^{*}=\left\langle\psi_{k}^{(0)}\left|O^{\dagger}\right| \psi_{n}^{(0)}\right\rangle$. La ecuación (5.39) es así la expresión para las Tensiones de Oscilador Generalizado para un operador arbitrario de un electrón, desarrollado a primer orden para el caso donde hay presente un campo electromagnético estático.

El análogo a las regla de suma de Bethe se obtiene sumando la ec. (5.39) sobre todos los estados excitados $n$. Así notando que los índices $k, n$ son índices mudos sobre la sumatoria, la suma de los dos primeros términos entre llaves se puede combinar para dar

$$
\sum_{k, n}\left\{\frac{O_{0 n}^{*} \mathbf{L}_{n k} O_{k 0}}{E_{n k}^{(0)}}+\frac{O_{0 k}^{*} \mathbf{L}_{k n} O_{n 0}}{E_{n k}^{(0)}}\right\} E_{n 0}^{(0)}=\sum_{k, n} O_{0 n}^{*} \mathbf{L}_{n k} O_{k 0} .
$$

Esta suma no está restringida debido a que los elementos diagonales de $\mathbf{L}$ son cero para un estado base no-degenerado. El tercer y cuarto término son un poco más complicados de manejar, pero usando la completez de las soluciones a orden cero (soluciones no perturbadas) uno obtiene que

$$
\begin{aligned}
& \sum_{k, n} \quad\left\{\frac{O_{0 n}^{*} O_{n k} \mathbf{L}_{k 0}}{E_{0 k}^{(0)}}+\frac{\mathbf{L}_{0 k} O_{k n}^{*} O_{n 0}}{E_{0 k}^{(0)}}\right\} E_{n 0}^{(0)}= \\
& =\sum_{k} \frac{1}{E_{0 k}^{(0)}}\left\{\mathbf{L}_{0 k}\left(O^{\dagger}\left[H^{(0)}, O\right]\right)_{k 0}-\left(\left[H^{(0)}, O^{\dagger}\right] O\right)_{0 k} \mathbf{L}_{k 0}\right\} .
\end{aligned}
$$

Lo anterior se satisface para cualquier operador de un electrón. Para simplificar aún más, y además concentrarnos en la regla de suma de Bethe, puesto que nuestro principal interes está en las Tensiones de Oscilador Generalizado, escogemos a $O=$ $\sum_{l} e^{-i \mathbf{q} \cdot \mathbf{r}_{l}}$, que usando los resultados anteriores y definiendo a

$$
M_{0 n}=\left\langle\psi_{0}^{(0)}\left|\sum_{l} e^{-i \mathbf{q} \cdot \mathbf{r}_{l}}\right| \psi_{n}^{(0)}\right\rangle
$$

$\mathrm{y}$

$$
P_{k 0}=\left(\sum_{l} e^{i \mathbf{q} \cdot \mathbf{r}_{l}}\left[H^{(0)}, \sum_{m} e^{-i \mathbf{q} \cdot \mathbf{r}_{m}}\right]\right)_{k 0}
$$


la regla de suma de Bethe se reduce a primer orden en la perturbación a

$$
\begin{aligned}
& \sum_{n} \frac{2 E_{n 0}}{q^{2}}\left|\left\langle 0\left|\sum_{l} e^{-i \mathbf{q} \cdot \mathbf{r}_{l}}\right| n\right\rangle\right|^{2}=\sum_{n} \frac{2 E_{n 0}^{(0)}}{q^{2}} M_{0 n} M_{0 n}^{*}+ \\
& +\quad \frac{e}{m q^{2}} \mathbf{B} \cdot\left\{\sum_{k, n} M_{0 n}^{*} \mathbf{L}_{n k} M_{0 k}+\sum_{k} \frac{1}{E_{0 k}^{(0)}}\left\{\mathbf{L}_{0 k} P_{k 0}+P_{0 k}^{\dagger} \mathbf{L}_{k 0}\right\}\right\} .
\end{aligned}
$$

El primer término (el término a orden cero) es justamente el término estandard, o el de campo libre [ec. (5.2)] y así éste es igual al número de electrones en el sistema.

El segundo término se puede simplificar usando la relación de completez para las eigenfunciones no-perturbadas y la relación

$$
\left[\mathbf{p}_{j}, e^{-i \mathbf{q} \cdot \mathbf{r}_{k}}\right]=-\hbar \mathbf{q} e^{-i \mathbf{q} \cdot \mathbf{r}_{k}} \delta_{j k},
$$

se puede demostrar que para este operador en particular, en la ec. (5.40), el siguiente término se reduce a

$$
\begin{aligned}
\sum_{k, n} M_{0 n}^{*} \mathbf{L}_{n k} M_{0 k}= & -\hbar\left\langle\psi_{0}^{(0)}\left|\sum_{j, k} e^{i \mathbf{q} \cdot\left(\mathbf{r}_{j}-\mathbf{r}_{k}\right)} \mathbf{r}_{k} \times \mathbf{q}\right| \psi_{0}^{(0)}\right\rangle \\
& +\left\langle\psi_{0}^{(0)}\left|\sum_{j, k, l} e^{i \mathbf{q} \cdot\left(\mathbf{r}_{j}-\mathbf{r}_{l}\right)} \mathbf{r}_{k} \times \mathbf{p}_{k}\right| \psi_{0}^{(0)}\right\rangle,
\end{aligned}
$$

donde el primer término es el valor de expectación del momento angular transferido en presencia del campo. Usando las relaciones siguientes

$$
\begin{gathered}
{\left[H^{(0)}, e^{\left. \pm i \mathbf{q} \cdot \mathbf{r}_{l}\right]}=\frac{\hbar^{2}}{2 m} e^{ \pm i \mathbf{q} \cdot \mathbf{r}_{l}}\left(q^{2} \mp 2 i \mathbf{q} \cdot \nabla_{l}\right),\right.} \\
{\left[H^{(0)}, \mathbf{r}_{l}\right]=-\frac{i \hbar}{m} \mathbf{p}_{l},} \\
{\left[e^{i \mathbf{q} \cdot \mathbf{r}_{p}}, \mathbf{r}_{j} \times \mathbf{p}_{j}\right]=-\hbar e^{i \mathbf{q} \cdot \mathbf{r}_{p}} \mathbf{r}_{j} \times \mathbf{q} \delta_{j p},}
\end{gathered}
$$

y

$$
\left[\mathbf{R} \cdot \mathbf{r}_{l}, \mathbf{L}_{j}\right]=i \hbar \mathbf{r}_{l} \times \mathbf{R} \delta_{l j},
$$

para un operador vectorial arbitrario $\mathbf{R}$ tal que $\left[\mathbf{R}, \mathbf{L}_{l}\right]=0$, que son faciles de demostrar, con ésto, se obtiene en la ec. (5.44) que

$$
\begin{aligned}
\sum_{k} \frac{1}{E_{0 k}^{(0)}}\left\{\mathbf{L}_{0 k} P_{k 0}+P_{0 k}^{\dagger} \mathbf{L}_{k 0}\right\}= & \hbar\left\langle\psi_{0}^{(0)}\left|\sum_{j, k} e^{i \mathbf{q} \cdot\left(\mathbf{r}_{j}-\mathbf{r}_{k}\right)} \mathbf{r}_{k} \times \mathbf{q}\right| \psi_{0}^{(0)}\right\rangle \\
& -\left\langle\psi_{o}^{(0)}\left|\sum_{j, k, l} e^{i \mathbf{q} \cdot\left(\mathbf{r}_{j}-\mathbf{r}_{l}\right)} \mathbf{r}_{k} \times \mathbf{p}_{k}\right| \psi_{0}^{(0)}\right.
\end{aligned}
$$


Como se puede ver, este es el negativo de la ec. (5.46). Así, la contribución total a primer orden a la regla de suma de Bethe, la suma de las ecuaciones (5.46) y (5.49) es cero, en acuerdo con la demostración general dada por al ec. (5.28).

Sin embargo, en cálculos realistas, aún las funciones de onda a orden cero son aproximadas pues se tiene un conjunto de base finito, así la cancelación exacta a la que conduce la ec. (5.28) no puede obtenerse. De ésto vemos que podemos usar como condición de calidad y consistencia de la base mediante la relación de desviación

$$
\sum_{k, n} M_{0 n}^{*} \mathbf{L}_{n k} M_{0 k}+\sum_{k} \frac{1}{E_{0 k}^{(0)}}\left\{\mathbf{L}_{0 k} P_{k 0}+P_{0 k}^{\dagger} \mathbf{L}_{k 0}\right\}=0,
$$

como una medida de la completez de la base. El primer término puede ser expresado como el producto vectorial de $\mathbf{q}$ y momento dipolar de un estado base [vease la ec. (5.46)], mientras que el segundo término es el límite a energía cero de una función de respuesta lineal [107]. Así, tenemos dos criterios para juzgar la calidad de un conjunto base en presencia de un campo electromagnético externo:

- La regla de suma de Bethe en el caso de campo libre, se puede utilizar para probar la completez de la base usada en el cálculo de propiedades atómicas y moleculares en presencia de campos electromagnéticos.

- La corrección a primer orden en la perturbación en la presencia de un campo electromagnético es una medida de la completez de la base. 


\section{Capítulo 6}

\section{Conclusiones generales}

Dada la importancia de los efectos moleculares en la sección de frenamiento electrónico, principalmente debido a los enlaces moleculares y la necesidad de aplicación de un modelo que cubra un rango de velocidades más amplio para altas velocidades no relativistas como el modelo de Bethe, se ha propuesto la utilización de la teoría cinética y la aproximación de plasma local para la determinación de las energias de excitación. Para ésto, es necesario contar con un buén conjunto de orbitales moleculares que describan de manera razonable el enlace químico. Como lo demuestra esta tesis, los orbitales FSGO representan una buena opción en dicho análisis, dado que nos permiten obtener la contribución a la sección de frenamiento electrónico como contribuciones de los carozos, enlaces y pares solitarios. Otra característica de los orbitales FSGO, es que nos describen el proceso de pérdida de energía sin la necesidad de recurrir a ningún parámetro externo, por lo que nuestro modelo es autoconsistente.

Por otro lado, como el lector habrá notado, la comparación con resultados experimentales, ha aclarado la importancia de la contribución de la estructura electrónica del proyectil en la sección de frenamiento electrónico, principalmente en base a las excitaciones e ionizaciones que ocurren durante la colisión y que directamente involucran a los electrones del proyectil. En base a ésto se ha desarrollado un modelo teórico puramente cuántico basado en la primera aproximación de Born y en el que se encuentra que para velocidades bajas, dentro de la validez del modelo, se predice una contribución del 10 al $20 \%$ a la sección de frenamiento electrónico para el proyectil, éste es un resultado nuevo e interesante. Debemos resaltar aquí, que debido a que no se considera intercambio de carga, de entrada se separa la 
contribución a la pérdida de energía en la contribución del proyectil y del blanco, debido a las excitaciones que toman lugar en el sistema. Para el desarrollo del modelo se ha seguido prácticamente el mismo desarrollo propuesto originalmente por H. A. Bethe [7] agregándole una descripción autoconsistente de Thomas-Fermi para cálcular el número de electrones del proyectil como función de la velocidad mediante la implementación del criterio adiabático de Bohr. Otra característica del modelo es que bajo ciertas condiciones de aplicabilidad éste se reduce a resultados bién conocidos en la literatura, dándonos confianza de su validez. Entre estos resultados se resalta la importancia del papel que juegan las energías medias de excitación para el proyectil y blanco iónicos no necesariamente neutros.

Sin embargo, debemos tener cuidado al hacer conclusiones finales respecto al modelo aquí propuesto, puesto que éste es valido para velocidades no relativistas, además de no considerar en forma detallada los mecanismos de captura e intercambio electrónico entre el proyectil y el blanco, debido principalmente al proceso dinámico en el que ésto toma lugar, además de la complejidad de un problema de $\mathrm{N}$-cuerpos. Aunque estos procesos son importantes y deben ser considerados en un tratamiento aún más general de la sección de frenamiento electrónico, creemos que las predicciones y resultados de nuestro método son muy útiles para el estudio de la pérdida de energía de iones con estructura electrónica.

Por otro lado, dada la importancia de la descripción del espectro de excitacion en el blanco y proyectil, es imperante conocer la estructura electrónica del sistema para obtener una buena descripción de las Tensiones de Oscilador Generalizado, pues éstos nos describen de manera general el proceso de transferencia de energía al sistema. Con ésto en mente, se demuestra que en el modelo RPA (o mejor conocido como teoría de Hartree-Fock dependiente del tiempo) la regla de suma de Bethe se satisface para una base completa. Esto nos sugiere que la descripción de la pérdida de energía en la aproximación RPA será adecuada. Hay que tener en cuenta que debido a que computacionalmente el tamaño de las bases utilizadas en química cuántica tienen una limitación (base incompleta), la regla de suma de Bethe solo se satisfacerá para la región de bajas transferencias de ímpetu q. Sin embargo, es esta región la que más contribuye a la sección de frenamiento [97].

En base a este trabajo surgió la necesidad de mostrar que esta propiedad importante se satisface también en presencia de campos electromagnéticos. Como se 
demuestra en esta tesis el resultado es, que en efecto, la regla de Bethe se satisface en presencia de campos electromagnéticos, dándonos la seguridad de que la descripción electrónica de moléculas en la presencia de campos eléctricos y magnéticos es adecuada para describir las Tensiones de Oscilador Generalizados.

Como vemos, el problema de la pérdida de energía está aún abierto a la investigación, tanto desde el punto de vista teórico como experimental dada la cantidad e importancia de los procesos que involucra su tratamiento, siendo uno de estos el estudio del efecto de estado de fase del blanco, puesto que la estructura electrónica del blanco cambia dependiendo de su estado de agregación, y así, la pérdida de energía es fuertemente dependiente de ésto, principalmente a velocidades intermedias en la curva de frenamiento. Esta es una de nuestras líneas de investigación actual. 


\section{Apéndice A}

\section{Teoría de Bethe estandard}

La teoría de Bethe para el frenamiento electrónico esta basada en la primera aproximación de Born [7, 67]. La idea fundamental detrás del tratamiento de Bethe es el uso de la transferencia de ímpetu del proyectil a los electrones del blanco como el factor dinámico en el proceso de pérdida de energía. Primeramente nos enfocaremos en esbozar la derivación de la fórmula de frenamiento siguiendo muy de cerca el tratamiento convencional y posteriormente deduciremos sus extensiones y modificaciones.

Consideremos un proceso en el cual un ion que se mueve con veocidad $v$ (en el sistema de laboratorio), masa $M_{1}$ y carga nuclear $Z_{1}$ e colisiona con un blanco estacionario de masa $M_{2}$ con $N_{2}$ electrones ligados en un estado inicial denotado por el ket $\left|m_{o}\right\rangle$. El proyectil es dispersado dentro del elemento de ángulo sólido $d \Omega$ a lo largo de la dirección con ángulos polares $(\theta, \psi)$ medidos desde el sistema de laboratorio. La sección diferencial de dispersión resulta

$$
\frac{d \sigma_{n 0}}{d \Omega}=\frac{(2 \pi)^{4} M_{p}^{2} k}{\hbar^{4} k_{0}}\left|\left\langle k m\left|H_{i n t}\right| k_{0} m_{0}\right\rangle\right|^{2}
$$

con $|m k\rangle$ el estado final del proyectil y blanco al que se originó la transición con $\hbar k$ el ímpetu final del proyectil al ser dispersado dentro del ángulo $d \Omega$ y donde el Hamiltoniano de interacción $H_{\text {int }}$ está dado por

$$
H_{\text {int }}=\sum_{j=1} \frac{Z_{1} Z_{2} e^{2}}{R}-Z_{1} e^{2} \sum_{j=1}^{N_{2}} \frac{1}{\left|\mathbf{R}-\mathbf{r}_{j}\right|},
$$

donde el primer término es la interacción Coulombiana entre el proyectil y los núcleos del blanco y el segundo término es la interacción entre los electrones del 
blanco y el proyectil. Esta expresión se obtiene como consecuencia de la regla de Oro de Fermi puesto que describe la probabilidad de transición del estado inicial al estado final por unidad de tiempo. Aquí los estados $|m k\rangle$ son productos de funciones de onda del proyectil $|k\rangle$ y de los estados electrónicos del blanco $|m\rangle$, i.e.

$$
|m k\rangle=\frac{1}{(2 \pi)^{3 / 2}} e^{i \mathbf{k} \cdot \mathbf{R}}|m\rangle
$$

Ahora, del elemento de matriz de la ecuación (A.1) se ve que el núcleo no contribuye puesto que las funciones de onda representan estados electrónicos y no nucleares. Así, usando transformada de Fourier en el Hamiltoniano de interacción el elemento de matriz se puede reescribir como

$$
\left\langle m k\left|H_{i n t}\right| m_{0} k_{0}\right\rangle=-\frac{4 \pi Z_{1} e^{2}}{(2 \pi)^{3} q^{2}}\left\langle m\left|\sum_{i=1}^{N_{2}} e^{i \mathbf{q} \cdot \mathbf{r}_{i}}\right| m_{0}\right\rangle
$$

donde $\hbar \mathbf{q}=\hbar\left(\mathbf{k}-\mathbf{k}_{0}\right)$ es el ímpetu transferido durante la colisión.

Para obtener la sección de frenamiento electrónico $S_{e}$, sumamos sobre todos los estados excitados $m$ e integramos ésto sobre todos los ángulos de dispersión, tal que

$$
S_{e}=-\frac{1}{n_{2}} \frac{d E}{d x}=\sum_{m>m_{0}} \int\left(E_{m}-E_{m_{0}}\right) \frac{d \sigma_{m m_{0}}}{d \Omega} d \Omega
$$

donde $n_{2}$ es el número de átomos por unidad de volumen del blanco, $d E / d x$ es el poder de frenamiento y $E_{m}-E_{m_{0}}$ es la energía transferida por el proyectil, que por conservación de energía es la energía necesaria para una transición del estado inicial $\left|m_{0}\right\rangle$ al estado final $|m\rangle$.

Si los átomos del medio no tienen orientación preferencial, cualquier dependencia del elemento de matriz sobre la dirección de q desaparecerá después de sumar sobre aquellos valores de $m$ que pertencen a un valor de $E_{m}$ dado, i.e.

$$
d \Omega=\frac{2 \pi q d q}{k k_{0}}
$$

Así, la sección de frenamiento electrónico es

$$
S_{e}=\frac{8 \pi Z_{1}^{2} e^{4}}{\hbar^{2} v^{2}} \sum_{m>m_{0}} \int \frac{d q}{q^{3}}\left(E_{m}-E_{m_{0}}\right)\left|\left\langle m\left|\sum_{j=1}^{N_{2}} e^{-i \mathbf{q} \cdot \mathbf{r}_{j}}\right| m_{0}\right\rangle\right|^{2}
$$


Antes de continuar, resulta útil el introducir la definición de las Tensiones de Oscilador Generalizado (GOS) $F_{m m_{0}}$ para una transición del estado 0 al estado $n$

$$
F_{m m_{0}}(\mathbf{q})=\frac{2 m}{\hbar^{2} q^{2}}\left(E_{m}-E_{m_{0}}\right)\left|\left\langle m\left|\sum_{i=1}^{N_{2}} e^{i \mathbf{q} \cdot \mathbf{r}_{i}}\right| m_{0}\right\rangle\right|^{2}
$$

las cuales son una medida de la densidad de probabilidad de varias pérdidas de energía $E_{m}-E_{m_{0}}$ para un ímpetu transferido q. En el Apéndice $\mathrm{C}$ se discute brevemente por qué a las cantidades $F_{m m_{0}}$ se les conoce como GOS.

Con esta definición, la sección de frenamiento electrónico se puede escribir como

$$
S_{e}=\frac{8 \pi Z_{1}^{2} e^{4}}{\hbar^{2} v^{2}} \sum_{m>m_{0}} \int \frac{d q}{q} F_{m m_{0}}(\mathbf{q}),
$$

Los límites de integración en la ec. (A.9) están determinados por la cinemática del proceso de colisión. El mínimo valor de integración se calcula suponiendo que hay al menos una excitación en el blanco, así $q_{\min }=\left(E_{m}-E_{m_{0}}\right) / \hbar v$. El máximo ímpetu transferido se calcula a partir de la conservación del ímpetu y de la energía, que suponiendo que el proyectil se mueve con velocidades mayores que la de los electrones del blanco se obtiene, $q_{\max }=2 m_{e} v / \hbar$. Como se ve de estos límites de integración, el mínimo ímpetu trasferido depende del estado de excitación $n$. De acuerdo a Bethe, para que la aproximación de Born sea válida, uno se debe de restringir a la región de velocidades $v>>v_{e}$ donde $v_{e}$ es la velocidad de los electrones del blanco. Con esta suposición, la integral sobre $q$ se puede separar en dos contribuciones: bajas y altas transferencias de ímpetu, i.e.

$$
S_{e, b}=\frac{4 \pi Z_{1}^{2} e^{4}}{m v^{2}} \sum_{m>m_{0}} \int_{q_{m i n}}^{\epsilon / \hbar v} \frac{d q}{q} F_{m m_{0}}(\mathbf{q})
$$

y

$$
S_{e, a}=\frac{4 \pi Z_{1}^{2} e^{4}}{m v^{2}} \sum_{m>m_{0}} \int_{\epsilon / \hbar v}^{q_{\max }} \frac{d q}{q} F_{m m_{0}}(\mathbf{q}),
$$

donde $\epsilon$ es una energía intermedia característica del sistema. La segunda integral se puede llevar a cabo inmediatamente. Puesto que los límites no dependen del estado excitado $n$, podemos intercambiar la suma con la integral y haciendo uso de la regla de suma de Bethe (véase el Apéndice C)

$$
\sum_{m} F_{m m_{0}}(\mathbf{q})=N_{2}
$$


la cual nos garantiza la completez de las funciones de onda del sistema, obtenemos que:

$$
S_{e_{a}}=\frac{4 \pi Z_{1}^{2} e^{4}}{m v^{2}} N_{2} \ln \frac{2 m v^{2}}{\epsilon}
$$

Por otro lado, para calcular la primera integral notamos que para $v>>v_{e}$, los límites de integración son muy pequeños, i.e. bajas transferencias de ímpetu, la contribución principal provendrá de la aproximación dipolar de las Tensiones de Oscilador Generalizado

$$
f_{m m_{0}}=\lim _{q \rightarrow 0} F_{m m_{0}}(q) \quad,
$$

donde $f_{m m_{0}}$ son las Tensiones de Oscilador Dipolar (DOS) (véase el Apendice C) las cuales son independientes de q. Así,

$$
S_{e_{b}}=\frac{4 \pi Z_{1}^{2} e^{4}}{m v^{2}} \ln \frac{\epsilon}{\left(E_{m}-E_{m_{0}}\right)}
$$

y definiendo la energía media de excitación $I_{2}$ del blanco como

$$
N_{2} \ln I_{2}=\sum_{m} f_{m m_{0}} \ln \left(E_{m}-E_{m_{0}}\right) \quad,
$$

con lo que $S_{e_{b}}$ se reduce a

$$
S_{e_{b}}=\frac{4 \pi Z_{1}^{2} e^{4}}{m v^{2}} \ln \frac{\epsilon}{I_{2}}
$$

donde se ha hecho uso de nuevo de la regla de suma de Bethe para el caso dipolar que se conoce como la regla de suma de Thomas-Reiche-Kuhn (TRK).

Finalmente se obtiene la sección de frenamiento electrónico como

$$
S_{e}(v)=\frac{4 \pi Z_{1}^{2} e^{4}}{m v^{2}} \ln \frac{2 m v^{2}}{I_{2}}
$$

Esta expresión es válida para altas velocidades no relativistas bajo la consideración de que el proyectil no tiene estructura electrónica. 


\section{Apéndice B}

\section{Simplificación algebraica de $S_{e}(v)$}

Para obtener la ecuación (3.23) partimos de hecho que necesitamos el cuadrado del elemento de matriz (3.21) dado por

$$
\begin{aligned}
\left\langle n m k\left|\hat{H}_{i n t}\right| n_{0} m_{0} k_{0}\right\rangle= & \frac{2 e^{2}}{(2 \pi)^{2} q^{2}}\left[Z_{12} T_{m m_{0}}(\mathbf{q}) \delta_{n n_{0}}\right. \\
& \left.+Z_{2}{ }_{1} T_{n n_{0}}(\mathbf{q}) \delta_{m m_{0}}-{ }_{1} T_{n n_{0}}(\mathbf{q})_{2} T_{m m_{0}}(\mathbf{q})\right]
\end{aligned}
$$

el cual aparece en la sección de frenamiento electrónico $S_{e}(v)$, la cual de acuerdo a la ec. (3.19) es

$$
S_{e}(v)=\frac{(2 \pi)^{4} M_{1}^{2}}{\hbar^{4}} \sum_{n, m} \int\left(E_{n n_{0}}+E_{m m_{0}}\right)\left|\left\langle n m k\left|\hat{H}_{i n t}\right| n_{0} m_{0} k_{0}\right\rangle\right|^{2} \frac{k}{k_{0}} d \Omega
$$

así, sustituyendo la ec. (B.1) en la ecuación anterior, obtenemos

$$
\begin{aligned}
S_{e}(v)= & \frac{4 e^{4} M_{1}^{2}}{\hbar^{4}} \sum_{n, m} \int\left(E_{n n_{0}}+E_{m m_{0}}\right) \mid Z_{12} T_{m m_{0}}(\mathbf{q}) \delta_{n n_{0}} \\
& +Z_{12} T_{n n_{0}}(\mathbf{q}) \delta_{m m_{0}}-\left.{ }_{1} T_{n n_{0}}(\mathbf{q})_{2} T_{m m_{0}}(\mathbf{q})\right|^{2} \frac{k}{k_{0}} d \Omega
\end{aligned}
$$

y que utilizando un procedimiento similar al usado para deducir la ec. (A.7) i.e. , si los átomos del medio no tienen una orientación preferencial, cualquier dependencia del elemento de matriz sobre la dirección de q desaparecerá despues de sumar sobre aquellos valores de $m$ y $n$ que pertenecen a un determinado valor de $E_{n n_{0}}+E_{m m_{0}}$ dado, por lo que

$$
d \Omega=\frac{2 \pi q d q}{k k_{0}}
$$


y usando el hecho de que $\hbar k_{0}=M_{1} v$ obtenemos que

$$
\begin{aligned}
S_{e}(v)= & \frac{8 \pi e^{4}}{\hbar^{2} v^{2}} \sum_{n, m} \int\left(E_{n n_{0}}+E_{m m_{0}}\right) \mid Z_{12} T_{m m_{0}}(\mathbf{q}) \delta_{n n_{0}} \\
& +Z_{2}{ }_{1} T_{n n_{0}}(\mathbf{q}) \delta_{m m_{0}}-\left.{ }_{1} T_{n n_{0}}(\mathbf{q})_{2} T_{m m_{0}}(\mathbf{q})\right|^{2} \frac{d q}{q^{3}}
\end{aligned}
$$

Ahora, notemos que al reescribir la ecuación anterior en la siguiente forma

$$
\begin{aligned}
S_{e}(v)= & \frac{8 \pi e^{4}}{\hbar^{2} v^{2}} \sum_{n, m} \int\left\{E_{n n_{0}} \mid Z_{12} T_{m m_{0}}(\mathbf{q}) \delta_{n n_{0}}+Z_{21} T_{n n_{0}}(\mathbf{q}) \delta_{m m_{0}}\right. \\
& -\left.{ }_{1} T_{n n_{0}}(\mathbf{q}){ }_{2} T_{m m_{0}}(\mathbf{q})\right|^{2}+E_{m m_{0}} \mid Z_{12} T_{m m_{0}}(\mathbf{q}) \delta_{n n_{0}}+Z_{21} T_{n n_{0}}(\mathbf{q}) \delta_{m n}(\mathrm{~B} .6) \\
& \left.-\left.{ }_{1} T_{n n_{0}}(\mathbf{q})_{2} T_{m m_{0}}(\mathbf{q})\right|^{2}\right\} \frac{d q}{q^{3}}
\end{aligned}
$$

el primer término desaparece cuando $n=n_{0}$ puesto que $E_{n n_{0}}=0$ i.e. , sólo contribuyen aquellos para los cuales $n \neq n_{0}$; de la misma manera el segundo término solo contruye para $m \neq m_{0}$. Así en el primer término desaparece el primer sumando debido a la delta de Kronecker. Lo mismo sucede para el segundo sumando en el término con $E_{m m_{0}}$. T omando ésto en cuenta tenemos que

$$
\begin{aligned}
S_{e}(v)= & \frac{8 \pi e^{4}}{\hbar^{2} v^{2}} \sum_{n, m} \int\left\{E_{n n_{0}}\left|{ }_{1} T_{n n_{0}}(\mathbf{q})\right|^{2}\left|Z_{2} \delta_{m m_{0}}-{ }_{2} T_{m m_{0}}(\mathbf{q})\right|^{2}\right. \\
& \left.+E_{m m_{0}}\left|{ }_{2} T_{m m_{0}}(\mathbf{q})\right|^{2}\left|Z_{1} \delta_{n n_{0}}-{ }_{1} T_{n n_{0}}(\mathbf{q})\right|^{2}\right\} \frac{d q}{q^{3}} .
\end{aligned}
$$

Introduciendo la definición de las Tensiones de Oscilador generalizado para el proyectil y blanco i.e. , usando

$$
\begin{aligned}
{ }_{1} F_{n n_{0}}(\mathbf{q}) & =\left.\left.\frac{2 m_{e}}{\hbar^{2} q^{2}} E_{n n_{0}}\right|_{1} T_{n n_{0}}(\mathbf{q})\right|^{2} \\
{ }_{2} F_{m m_{0}}(\mathbf{q}) & =\left.\left.\frac{2 m_{e}}{\hbar^{2} q^{2}} E_{m m_{0}}\right|_{2} T_{m m_{0}}(\mathbf{q})\right|^{2}
\end{aligned}
$$

vemos que la ec. (B.7) se puede escribir como

$$
\begin{aligned}
S_{e}(v)=\frac{4 \pi e^{4}}{m_{e} v^{2}} \sum_{n, m} \int & \left\{{ }_{1} F_{n n_{0}}(\mathbf{q})\left|Z_{2} \delta_{m m_{0}}-{ }_{2} T_{m m_{0}}(\mathbf{q})\right|^{2}\right. \\
+ & \left.{ }_{2} F_{m m_{0}}(\mathbf{q})\left|Z_{1} \delta_{n n_{0}}-{ }_{1} T_{n n_{0}}(\mathbf{q})\right|^{2}\right\} \frac{d q}{q} .
\end{aligned}
$$


que es la ecuación (3.23). 


\section{Apéndice $\mathrm{C}$}

\section{Tensiones de Oscilador Generalizado}

Queremos probar la regla de suma de Bethe [ec. 5.2] para las tensiones de oscilador generalizado, i.e. queremos demostrar

$$
\sum_{m} F_{m m_{0}}(\mathbf{q})=N
$$

donde $N$ es el número de electrones del sistema y

$$
F_{m m_{0}}(\mathbf{q})=\frac{2 m_{e}}{\hbar^{2} q^{2}}\left(E_{m}-E_{m_{0}}\right)\left|\left\langle m\left|\sum_{j=1}^{N} e^{-i \mathbf{q} \cdot \mathbf{r}_{j}}\right| m_{0}\right\rangle\right|^{2} .
$$

Usando el hecho de que el conjunto de eigenfunciones del Hamiltoniano forman un conjunto completo, i.e.

$$
\sum_{m}|m\rangle\langle m|=1
$$

obtenemos

$$
\sum_{m} F_{m m_{0}}(\mathbf{q})=\frac{2 m_{e}}{\hbar^{2} q^{2}}\left\langle m_{0}\left|\sum_{j}^{N} e^{-i \mathbf{q} \cdot \mathbf{r}_{j}}\left[H, \sum_{k}^{N} e^{i \mathbf{q} \cdot \mathbf{r}_{k}}\right]\right| m_{0}\right\rangle .
$$

Introduciendo la expresión explícita para el Hamiltoniano, podemos evaluar facilmente el conmutador $\left[H, \sum_{j}^{N} e^{i \mathbf{q} \cdot \mathbf{r}_{j}}\right]$. Como

$$
H=\sum_{i}^{N} \frac{p^{2}}{2 m_{e}}+\psi\left(\mathbf{r}_{1}, \cdots, \mathbf{r}_{N}\right)
$$

donde la primera parte es el operador de energía cinética y la segunda corresponde a la interacción Coulombiana entre los electrones y el núcleo y la interacción electrón- 
electrón. De ésto obtenemos que

$$
\left[H, \sum_{j}^{N} e^{i \mathbf{q} \cdot \mathbf{r}_{j}}\right]=\frac{1}{2 m_{e}} \sum_{j}^{N}\left[\mathbf{p}_{j}^{2}, e^{i \mathbf{q} \cdot \mathbf{r}_{j}}\right]
$$

y utilizando la regla de conmutación

$$
[A B, C]=[A, C] B+A[B, C]
$$

y el hecho de que

$$
\left[\mathbf{p}_{j}, e^{i \mathbf{q} \cdot \mathbf{r}_{j}}\right]=\hbar \mathbf{q} e^{-i \mathbf{q} \cdot \mathbf{r}_{j}}
$$

con lo que

$$
\left[H, \sum_{j}^{N} e^{i \mathbf{q} \cdot \mathbf{r}_{j}}\right]=\frac{1}{2 m_{e}} \sum_{j}^{N} e^{i \mathbf{q} \cdot \mathbf{r}_{j}}\left(\hbar^{2} q^{2}+2 \hbar \mathbf{q} \cdot \mathbf{p}_{j}\right)
$$

tal que la regla de suma [ec. (C.4)] se puede escribir como

$$
\begin{aligned}
\sum_{m} F_{m m_{0}}(\mathbf{q})= & \sum_{j=1}^{N}\left\langle m_{0} \mid m_{0}\right\rangle+\frac{2}{\hbar q^{2}} \mathbf{q} \cdot\left\langle m_{0}\left|\sum_{j}^{N} \mathbf{p}_{j}\right| m_{0}\right\rangle+ \\
& \frac{1}{\hbar^{2} q^{2}} \sum_{n \neq k}\left\langle m_{0}\left|e^{-i \mathbf{q} \cdot\left(\mathbf{r}_{k}-\mathbf{r}_{j}\right)}\left\{\hbar^{2} q^{2}+2 \hbar \mathbf{q} \cdot \mathbf{p}_{k}\right\}\right| m_{0}\right\rangle
\end{aligned}
$$

donde los dos primeros términos surgen de considerar el caso $j=k$. El primer término es el número de electrones $N$ debido a la normalización de las funciones de onda. Por otra parte, para calcular el segundo término, podemos relacionar el operador de momento con el conmutador entre el Hamiltoniano del sistema y el operador de posición como

$$
\begin{aligned}
{\left[H, \mathbf{r}_{l}\right] } & =\frac{1}{2 m_{e}} \sum_{k}\left[\mathbf{p}_{k}^{2}, \mathbf{r}_{l}\right] \\
& =-\frac{3 i \hbar}{m_{e}} \mathbf{p}_{l}
\end{aligned}
$$

Así, el segundo término en la ec. (C.10) es proporcional a $\left\langle m_{0}\left|\left[H, \sum_{l} \mathbf{r}_{l}\right]\right| m_{0}\right\rangle$, el cual es claramente cero, debido a que $\left|m_{0}\right\rangle$ es solución del estado base de la ecuación de Schödinger, con lo que la ec. (C.10) se reduce a

$$
\sum_{m} F_{m m_{0}}(\mathbf{q})=N+\frac{1}{\hbar^{2} q^{2}} \sum_{n \neq k}\left\langle m_{0}\left|e^{-i \mathbf{q} \cdot\left(\mathbf{r}_{k}-\mathbf{r}_{j}\right)}\left\{\hbar^{2} q^{2}+2 \hbar \mathbf{q} \cdot \mathbf{p}_{k}\right\}\right| m_{0}\right\rangle
$$


Si escribimos la función de onda explicitamente como $\phi_{m_{0}}(\mathbf{r})$ y usamos el hecho de que $\mathbf{p}_{l}=-i \hbar \nabla_{l}$, entonces el segundo término de la ecuación anterior se puede escribir como

$$
\begin{gathered}
\sum_{j \neq k}\left\langle m_{0}\left|e^{i \mathbf{q} \cdot\left(\mathbf{r}_{k}-\mathbf{r}_{j}\right)}\left\{\hbar^{2} q^{2}+2 \hbar \mathbf{q} \cdot \mathbf{p}_{k}\right\}\right| m_{0}\right\rangle= \\
i \hbar \mathbf{q} \cdot \sum_{j \neq k} \int d \tau e^{i \mathbf{q} \cdot\left(\mathbf{r}_{k}-\mathbf{r}_{j}\right)}\left[\left(\nabla_{k} \phi_{m_{0}}^{*}(\mathbf{r})\right) \phi_{m_{0}}(\mathbf{r})-\phi_{m_{0}}^{*}(\mathbf{r}) \nabla_{k} \phi_{m_{0}}(\mathbf{r})\right] .
\end{gathered}
$$

Para el tipo de Hamiltoniano no-relativista que estamos considerando aquí, la densidad de corriente para el k-ésimo electrón esta dada por

$$
\mathbf{J}_{k}^{0}=\frac{e \hbar}{2 i m_{e}}\left[-\left(\nabla_{k} \phi_{m_{0}}^{*}(\mathbf{r})\right) \phi_{m_{0}}(\mathbf{r})+\phi_{m_{0}}^{*}(\mathbf{r}) \nabla_{k} \phi_{m_{0}}(\mathbf{r})\right],
$$

el cual es precisamente el término entre paréntesis y así encontramos que

$$
\sum_{j \neq k}\left\langle m_{0}\left|e^{i \mathbf{q} \cdot\left(\mathbf{r}_{k}-\mathbf{r}_{j}\right)}\left\{\hbar^{2} q^{2}+2 \hbar \mathbf{q} \cdot \mathbf{p}_{k}\right\}\right| m_{0}\right\rangle=\frac{2 m_{e}}{e} \mathbf{q} \cdot \sum_{j \neq k} \int d \tau e^{i \mathbf{q} \cdot\left(\mathbf{r}_{k}-\mathbf{r}_{j}\right)} \mathbf{J}_{k}^{0} .
$$

Por otro lado, integrando por partes $\mathbf{J}_{k}^{0}$ encontramos que

$$
\int d \tau \mathbf{J}_{k}^{0}=\frac{e}{m_{e}}\left\langle m_{0}\left|\mathbf{p}_{k}\right| m_{0}\right\rangle
$$

y en conexión con el desarrollo de la ecuación (C.12) vemos que el término de la derecha de la ec. (C.16) es cero, y como $\mathbf{J}_{k}^{0}$ es un vector, implica que cada componente debe ser cero, con lo que la ec. (C.12) se reduce a

$$
\sum_{m} F_{m m_{0}}(\mathbf{q})=N
$$

que es la regal de suma de Bethe.

Notemos que esta demostración no supone que las eigenfunciones deban ser reales, por lo que la regla de suma se aplica a estados con eigenfunciones complejas, así como para estados degenerados.

En el caso de pequeñas transferencias de momento, las Tensiones de Oscilador Generalizado se reducen a

$$
f_{m m_{0}}=\lim _{q \rightarrow 0} F_{m m_{0}}(\mathbf{q})=\frac{2 m_{e}}{\hbar^{2} q^{2}}\left(E_{m}-E_{m_{0}}\right)\left|\mathbf{q} \cdot\left\langle m\left|\sum_{j}^{N} \mathbf{r}_{j}\right| m_{0}\right\rangle\right|^{2}
$$


donde hemos hechos uso de la expansión de la función exponencial para pequeños valores del argumento y debido a la ortonormalidad de las eigenfunciones solo retenos el primer orden de la expansión. De ésto parecería que el valor de la regla de suma de Bethe para pequeños valores de q depende de la dirección de este último, sin embargo notemos que el espacio es isotrópico, lo cual nos permite usar el hecho de que para cualquier vector unitario $\hat{\mathbf{q}}$

$$
\left|\left\langle m\left|\sum_{j}^{N} \hat{\mathbf{q}} \cdot \mathbf{r}_{j}\right| m_{0}\right\rangle\right|^{2}=\frac{1}{3}\left|\left\langle m\left|\sum_{j}^{N} \mathbf{r}_{j}\right| m_{0}\right\rangle\right|^{2}
$$

con lo que las Tensiones de Oscilador Generalizado para $q \rightarrow 0$ se reduce a

$$
f_{m m_{0}}=\frac{2 m_{e}}{3 \hbar^{2}}\left(E_{m}-E_{m_{0}}\right)\left|\left\langle m\left|\sum_{j}^{N} \mathbf{r}_{j}\right| m_{0}\right\rangle\right|^{2}
$$

que debido a la dependencia con $\mathbf{r}$ se conocen como las Tensiones de Oscilador Dipolar (DOS) y como vemos son independientes del momento transferido q.

El nombre de Tensiones de Oscilador Dipolar para las cantidades $f_{m m_{0}}$ se deriva de la expresión para la polarizabilidad de un átomo i.e. , un atomo sujeto a un campo eléctrico constante $E_{z}$ en la dirección del eje $z$ adquiere un momento dipolar inducido $\mu_{z}$ por lo que la polarizabilidad $\chi=\mu_{z} / E_{z}$ queda dada por la expresión

$$
\chi=2 e^{2} \sum_{n} \frac{\left|\left\langle n\left|\sum_{i=1}^{N} z_{i}\right| n_{0}\right\rangle\right|^{2}}{E_{n}-E_{n_{0}}}=\frac{\hbar^{2} e^{2}}{m_{e}} \sum_{n} \frac{f_{n n_{0}}}{E_{n}-E_{n_{0}}} .
$$




\section{Apéndice D}

\section{Regla de suma parcial}

Para derivar la ec. (3.27), la cual nos da la regla de suma parcial para la estructura electrónica del proyectil y del blanco, procedemos expandiendo dicha ecuación. Así,

$$
\begin{aligned}
& \sum_{n}\left|Z_{i} \delta_{n n_{0}}-{ }_{i} M_{n n_{0}}(\mathbf{q})\right|^{2}=\sum_{n}\left\{Z_{i} \delta_{n n_{0}}-Z_{i}{ }_{i} M_{n n_{0}}(\mathbf{q}) \delta_{n n_{0}}-Z_{i}{ }_{i} M_{n n_{0}}^{*}(\mathbf{q}) \delta_{n n_{0}}\right. \\
& \left.+{ }_{i} M_{n n_{0}}(\mathbf{q}){ }_{i} M_{n n_{0}}^{*}(\mathbf{q})\right\} \\
& =Z_{i}-Z_{i{ }_{i}} M_{n_{0} n_{0}}(\mathbf{q})-Z_{i}{ }_{i} M_{n_{0} n_{0}}^{*}(\mathbf{q}) \\
& +\sum_{n}{ }_{i} M_{n n_{0}}(\mathbf{q}){ }_{i} M_{n n_{0}}^{*}(\mathbf{q})
\end{aligned}
$$

donde $1=1,2$ (proyectil o blanco). Pero como

$$
\sum_{n}{ }_{i} M_{n n_{0}}(\mathbf{q}){ }_{i} M_{n n_{0}}^{*}(\mathbf{q})=\sum_{n}\left\langle n_{0}\left|\sum_{j=1}^{N} e^{-i \mathbf{q} \cdot \mathbf{r}_{j}}\right| n\right\rangle\left\langle n\left|\sum_{k=1}^{N} e^{i \mathbf{q} \cdot \mathbf{r}_{k}}\right| n_{0}\right\rangle
$$

podemos simplificar utilizando la relación de completez de la base, i.e.

$$
\sum_{n}|n\rangle\langle n|=1
$$

con lo que obtenemos

$$
\sum_{n}{ }_{i} M_{n n_{0}}(\mathbf{q}){ }_{i} M_{n n_{0}}^{*}(\mathbf{q})=\left\langle n_{0}\left|\sum_{j, k=1}^{N} e^{i \mathbf{q} \cdot\left(\mathbf{r}_{k}-\mathbf{r}_{j}\right.}\right| n_{0} \cdot\right\rangle
$$

Ahora, consideremos una función de onda de tipo Hartree para el estado base $\left|n_{0}\right\rangle$, i.e.

$$
\left|n_{0}\right\rangle=\phi_{1} \phi_{2} \cdots \phi_{N}
$$


con ésto, encontramos que el elemento de matriz (D.4) se reduce a

$$
\left\langle n_{0}\left|\sum_{j, k=1}^{N} e^{i \mathbf{q} \cdot\left(\mathbf{r}_{k}-\mathbf{r}_{j}\right.}\right| n_{0}\right\rangle=\sum_{k=1}^{N}\left\langle\phi_{k}\left|e^{i \mathbf{q} \cdot \mathbf{r}_{k}}\right| \phi_{k}\right\rangle \sum_{j=1}^{N}\left\langle\phi_{j}\left|e^{-i \mathbf{q} \cdot \mathbf{r}_{j}}\right| \phi_{j}\right\rangle
$$

Por otro lado, notemos que utilizando la función de onda (D.5) es fácil demostrar el siguiente resultado

$$
\begin{aligned}
{ }_{i} M_{n_{0} n_{0}}(\mathbf{q}) & =\left\langle n_{0}\left|\sum_{j=1}^{N} e^{-i \mathbf{q} \cdot \mathbf{r}_{j}}\right| n_{0}\right\rangle \\
& =\sum_{j=1}^{N}\left\langle\phi_{j}\left|e^{-i \mathbf{q} \cdot \mathbf{r}_{j}}\right| \phi_{j}\right\rangle
\end{aligned}
$$

con lo cual vemos que la ec. (D.4) se reduce a

$$
\sum_{n}{ }_{i} M_{n n_{0}}(\mathbf{q}){ }_{i} M_{n n_{0}}^{*}(\mathbf{q})={ }_{i} M_{n_{0} n_{0}}(\mathbf{q}){ }_{i} M_{n_{0} n_{0}}^{*}(\mathbf{q})
$$

Con lo que finalmente obtenemos el resultado deseado

$$
\begin{aligned}
\sum_{n}\left|Z_{i} \delta_{n n_{0}}-{ }_{i} M_{n n_{0}}(\mathbf{q})\right|^{2}= & Z_{i}-Z_{i{ }_{i}} M_{n_{0} n_{0}}(\mathbf{q})-Z_{i{ }_{i}} M_{n_{0} n_{0}}^{*}(\mathbf{q}) \\
& +{ }_{i} M_{n_{0} n_{0}}(\mathbf{q}){ }_{i} M_{n_{0} n_{0}}^{*}(\mathbf{q}) \\
= & \left|Z_{i}-{ }_{i} M_{n_{0} n_{0}}(\mathbf{q})\right|^{2}
\end{aligned}
$$

Q. E. D. 


\section{Apéndice E}

\section{Integración de $S_{e}$ para q pequeñas}

Derivamos la ecuación (3.30) mediante la suposición de que $\epsilon$ está definida tal que permita el intercambio de la integración con la sumatoria en la ec. (3.23) viz., para asegurar que

$$
\begin{aligned}
& \sum_{n, m}\left\{\int_{q_{\min }}^{q_{\max }}\left|Z_{2} \delta_{m_{0} m}-{ }_{2} T_{m_{0} m}(\mathbf{q})\right|^{2}{ }_{1} F_{n_{0} n}(\mathbf{q}) \frac{d \mathbf{q}}{q^{2}}\right. \\
& \left.+\int_{q_{\min }}^{q_{\max }}\left|Z_{1} \delta_{n_{0} n}-{ }_{1} T_{n_{0} n}(\mathbf{q})\right|^{2}{ }_{2} F_{m_{0} m}(\mathbf{q}) \frac{d \mathbf{q}}{q^{2}}\right\} \\
= & \left\{\int_{\epsilon / \hbar v}^{q_{\max }} \sum_{n, m}\left|Z_{2} \delta_{m_{0} m}-{ }_{2} T_{m_{0} m}(\mathbf{q})\right|^{2}{ }_{1} F_{n_{0} n}(\mathbf{q}) \frac{d \mathbf{q}}{q^{2}}\right. \\
& \left.+\int_{\epsilon / \hbar v}^{q_{\max }} \sum_{n, m}\left|Z_{1} \delta_{n_{0} n}-{ }_{1} T_{n_{0} n}(\mathbf{q})\right|^{2}{ }_{2} F_{m_{0} m}(\mathbf{q}) \frac{d \mathbf{q}}{q^{2}}\right\} .
\end{aligned}
$$

Esto puede re-escribirse como

$$
\begin{aligned}
& \sum_{n, m}\left\{\int_{\epsilon / \hbar v}^{\left(w_{n_{0} n}+w_{m_{0} m}\right) / v}\left|Z_{2} \delta_{m_{0} m}-{ }_{2} T_{m_{0} m}(\mathbf{q})\right|^{2}{ }_{1} F_{n_{0} n}(\mathbf{q}) \frac{d \mathbf{q}}{q^{2}}\right. \\
& \left.+\int_{\epsilon / \hbar v}^{\left(w_{n_{0} n}+w_{m_{0} m}\right) / v}\left|Z_{1} \delta_{n_{0} n}-{ }_{1} T_{n_{0} n}(\mathbf{q})\right|^{2}{ }_{2} F_{m_{0} m}(\mathbf{q}) \frac{d \mathbf{q}}{q^{2}}\right\}=0 .
\end{aligned}
$$

Ambos límites de integración son pequeños para velocidades más grandes que la velocidad orbital de los electrones del proyectil y blanco. Por lo tanto, usando la suposición de Bethe $\left(v>v_{e}\right)$ expandemos la ec. (E.1) para pequeñas transferencias 
de ímpetu. Así, en la aproximación dipolar obtenemos que

$$
\begin{aligned}
& \sum_{n, m}\left\{\int_{\left(w_{n_{0} n}+w_{m_{0} m}\right) / v}^{\epsilon / \hbar v}\left(Z_{1}-N_{1}\right)^{2} \delta_{n_{0} n}{ }_{2} f_{m_{0} m} \frac{d \mathbf{q}}{q^{2}}\right. \\
& \left.+\int_{\left(w_{n_{0} n}+w_{m_{0} m}\right) / v}^{\epsilon / \hbar v}\left(Z_{2}-N_{2}\right)^{2} \delta_{m_{0} m}{ }_{1} f_{n_{0} n} \frac{d \mathbf{q}}{q^{2}}\right\}=0,
\end{aligned}
$$

donde

$$
\begin{aligned}
{ }_{1} f_{n_{0} n} & =\frac{2 m_{e} w_{n_{0} n}}{\hbar}\left|\left\langle n\left|\sum_{i}^{N_{1}} \hat{\mathbf{q}} \cdot \mathbf{x}_{i}\right| n_{0}\right\rangle\right|^{2} \\
{ }_{2} f_{m_{0} m} & =\frac{2 m_{e} w_{m_{0} m}}{\hbar}\left|\left\langle m\left|\sum_{i}^{N_{2}} \hat{\mathbf{q}} \cdot \mathbf{y}_{i}\right| m_{0}\right\rangle\right|^{2}
\end{aligned}
$$

son las tensiones de oscilador dipolar y $\mathbf{q}$ es un vector unitario paralelo a q. Ahora, la integración sobre q se puede llevar a cabo inmediatamente debido a la independencia de q en las tensiones de oscilador dipolar, obteniéndose

$$
\sum_{m}\left(Z_{1}-N_{1}\right)_{2}^{2} f_{m_{0} m} \ln \left(\frac{\epsilon}{\hbar w_{m_{0} m}}\right)+\sum_{n}\left(Z_{2}-N_{2}\right)^{2}{ }_{1} f_{n_{0} n} \ln \left(\frac{\epsilon}{\hbar w_{n_{0} n}}\right)=0 .
$$

Usando la definición standard de la energía media de excitación [72]

$$
\ln _{i} I_{0}=\frac{1}{N_{e, i}} \sum_{s} f_{s_{0} s} \ln \left(\hbar w_{s_{0} s}\right) \quad i=1,2
$$

donde la suma es sobre los estados excitados, y sustituyendo ésto en la ec. (E.3) obtenemos

$$
N_{2}\left(Z_{1}-N_{1}\right)^{2} \ln \left(\frac{\epsilon}{{ }_{2} I_{0}}\right)+N_{1}\left(Z_{2}-N_{2}\right)^{2} \ln \left(\frac{\epsilon}{{ }_{1} I_{0}}\right)=0
$$

Definiendo

$$
\alpha=\frac{\left(Z_{2}-N_{2}\right)^{2} N_{1}}{\left(Z_{1}-N_{1}\right)^{2} N_{2}}
$$

y resolviendo para $\epsilon$ resulta que

$$
\epsilon={ }_{2} I_{0}^{1 / 1+\alpha}{ }_{1} I_{0}^{\alpha / 1+\alpha} .
$$




\section{Bibliografía}

[1] J.J. Thomson, Phil. Mag. 23 (1912) 449

[2] E. Rutherford, Phil. Mag. 21 (1911) 669.

[3] H. Geiger y E. Marsden, Proc. Roy. Soc. A82, (1909) 495.

[4] H. Geiger y E. Marsden, Phil. Mag. 25 (1913) 604.

[5] N. Bohr, Philos. Mag. 25, (1913) 10.

[6] N. Bohr, Mat. Fys. Medd. Dan. Vid. Selsk. 18, No. 8 (1948).

[7] H. A. Bethe, Ann. Phys. (Leipzig) 5, (1930) 325.

[8] E. Fermi, Phys. Rev. 57 (1940) 485.

[9] L. Landau, J. Phys. USSR 8, (1944) 201.

[10] D. I. Thwaites, Nucl. Instr. and Meth. B69 (1992) 53 y referencias ahí contenidas.

[11] D. I. Thwaites, Radiat. Res. 95 (1983) 495.

[12] D. I. Thwaites, Nucl. Instr. and Meth. B27 (1987) 293.

[13] J. R. Sabin y J. Oddershede, Nucl. Instr. and Meth. B64 (1992) 678.

[14] W. A. Grant y J. Williams, Sci. Prog. Oxf. 63 (1976) 27.

[15] P. Sigmund, Phys. Rev. A26 (1982) 2497.

[16] J. Oddershede y J. R. Sabin, Phys. Rev. A35 (1987) 3283. 
[17] R. Cabrera-Trujillo, S. A: Cruz y J. Soullard, Nucl. Instr. and Meth. B93 (1994) 166.

[18] R. Cabrera-Trujillo, S. A. Cruz, Jens Oddershede y John R. Sabin, Phys. Rev. A55(4) (1997) 2864.

[19] R. Cabrera-Trujillo, J. R. Sabin y Jens Oddershede, (por enviarse a Int. J. of Quantum Chemestry).

[20] R. Cabrera-Trujillo, John. R. Sabin, Yngve Øhrn y J. Oddershede, Phys. Rev. A57, (1998) 3115.

[21] véase por ejemplo, R. F. Willson, K. R. Lang y D. E. Gary, Astrophysical Journal, 418(1) (1993), 490.

[22] W. H. Bragg y R. Kleeman, Phil. Mag. 10 (1918) 305.

[23] D. Powers, Acc. Chem. Res. 13 (1980) 433; Nucl. Instr. and Meth. B40/41 (1989) 324.

[24] R. Kreutz, W. Neuwirth y W. Pietsch, Phys. Rev. A22 (1980) 324.

[25] G. Reiter, E. Pfaff y G. Clausnitzer, Nucl. Instr. and Meth B51 (1990) 320.

[26] P. Bauer, W. Rössler y P. Mertens, Nucl. Ianstr. and Meth. B69 (1992) 46.

[27] véase, por ejemplo, L. H. Toburen, in Atomic and Molecular Data for Radiotherapy and Radiation Research, (International Atomic Energy Agency, Vienna, 1995), Chap. 2.

[28] E. Bonderup, "Penetration of charged particles through matter". Universidad de Aarhus, Dinamarca, 2a edición, 1981.

[29] H.A. Kramers, Physica 8, (1947) 401

[30] J. Lindhard y M. Scharff, K. Dan. Vidensk. Selsk. Mat-Fys. Medd. 27 no. 15 (1953)

[31] J. R. Sabin y J. Oddershede, Phys. Rev. A26 (1982) 3209. 
[32] J. Oddershede y J. R. Sabin, At. Data. Nucl. Data Tables 31 (1984) 275.

[33] J. L. Dehmer, M. Inokuti y R. P. Saxon, Phys. Rev. A12 (1975) 102; M. Inokuti, T. Baer y J. L. Dehmer, ibid. A17 (1978) 1229; M. Inokuti, J. L. Dehmer, T. Baer y J. D. Hanson, ibid. A23 (1981) 95.

[34] J. R. Sabin y J. Oddershede, Nucl. Instr. and Meth. B27 (1987) 280.

[35] D. E. Meltzer, J. R. Sabin y S. B. Trickey, Phys. Rev. A41 (1990) 220.

[36] E. Clementi y C. Roetti, At. Data Nucl. Data Tables 14 (1974) 177.

[37] A. A. Frost, Theor. Chim. Acta 18 (1970) 156, y referencias incluidas.

[38] S. Y. Chu y A. A. Frost, J. Chem. Phys. 54 (1971) 760.

[39] A. A. Frost, J. Phys. Chem. 72 (1968) 1289.

[40] A. A. Frost y R. A. Rouse, J. Am. Chem. Soc. 90 (1968) 1965.

[41] P. H. Blustin y J. W. Linnet, J. Chem. Soc. Faraday II 70 (1974) 274.

[42] S. Y. Chu y A. A. Frost, J. Chem. Phys. 54 (1971) 764.

[43] A. A. Frost, J. Chem. Phys. 47 (1967) 3714.

[44] J. Oddershede y J. R. Sabin, Nucl. Instr. and Meth. B42 (1989) 7.

[45] C. J. Tung y J. W. Baum, Radiat. Res. 119 (1989) 413.

[46] B. L. Jhanwar, W. J. Meath y J. C. F. MacDonald, Radiat. Res. 106 (1986) 288.

[47] G. D. Zeiss, W. J. Meath, J. C. F. MacDonald y D. J. Dawson, Radiat. Res. 70 (1977) 284.

[48] J. Geertsen, J. Oddershede y J. R. Sabin, Phys. Rev. A34 (1986) 1104.

[49] G. Reiter, H. Baumgart, N. Kniest, E. Pfaff y G. Clausnietser, Nucl. Instr. and Meth. B27 (1987) 287. 
[50] H. K. Reynolds, D. N. F. Dunbar, W. A. Wenzel y W. Whaling, Phys. Rev. 92 (1953) 742.

[51] J. T. Park y E. J. Zimmerman, Phys. Rev. 131 (1963) 1611.

[52] H. Bichsel y L. E. Porter, Phys. Rev. A25 (1982) 2499.

[53] R. A. Langley, Phys. Rev. B12 (1975) 3575.

[54] F. Bloch, Ann. Phys. (Leipzig) 16, 285 (1933).

[55] W. H. Barkas, Nuclear Research Emulsions-I. Techniques and Theory, Academic Press, New York, (1963).

[56] Y. K. Kim and K. Cheng, Phys. Rev. A22, (1980) 61.

[57] T. Kaneko, Phys. Rev. A 43, (1991) 4780.

[58] D. R. Bates and W. Griffing, Proc. Phys. Soc. London Sec. A 66, (1953) 961; 67, (1954) 663; 68, (1955) 90.

[59] G. H. Gillespie and M. Inokuti, Phys. Rev. A 22, (1980) 2430.

[60] W. Brandt and M. Kitagawa, Phys. Rev. B 25, (1982) 5631.

[61] G. Schiwietz and P. L. Grande, Nucl. Instrum. and Methods B 69, (1992) 10.

[62] G. Schiwietz and P. L. Grande, Nucl. Instrum. and Methods B 90, (1994) 10.

[63] A. C. Diz, Y. Örn, and J. R. Sabin, Nucl. Instrum. and Methods B 96, (1995) 633.

[64] A. Arnau, M. Penalba, and P. M. Echenique, Nucl. Instrum. and Methods B 69, (1992) 102 .

[65] A. Arnau and P. M. Echenique, Nucl. Instrum. and Methods B 42, (1989) 165.

[66] M. Moneta and J. Czerbniak, Nucl. Instrum. and Methods B 90, (1994) 67.

[67] M. Born, Z. Physik 38, (1926) 803. Este método parece haber sido empleado primero por Lord Rayleigh, Phil. Mag. 12, (1881) 81. 
[68] N. Bohr, Phys. Rev. 58, 654 (1940); 59, (1941) 270.

[69] U. Fano and A. R. P. Rau, Atomic Collisions and Spectra (Academic, New York, 1986).

[70] R. W. James, The Optical Principles of the Diffraction of X-rays, edited by L. Bragg (G. Bell and Sons Ltd., London, 1967), p. 109.

[71] H. H. Mikkelsen, J. Oddershede, J. R. Sabin, and E. Bonderup, Nucl. Instrum. and Methods B 100, (1995) 451.

[72] H. A. Bethe and R. Jackiw, Intermediate Quantum Mechanics (Benjamin / Cummings, Reading 1986).

[73] M. Inokuti, Rev. Mod. Phys. 43, (1971) 297.

[74] D. R. Bates, in Atomic and Molecular Processes, edited by D. R. Bates (Academic, New York, 1962), Chap. 14.

[75] N. F. Mott and H. S. W. Massey, The Theory of Atomic Collisions (Clarendon, Oxford, 1965), 3rd ed., p. 477.

[76] Y.S. Yarlagadda, J. E. Robinson, and W. Brandt, Phys. Rev. B 17, (1978) 3473.

[77] L. H. Thomas, Proc. Cambridge Philos. Soc. 23, (1927) 542; E. Fermi, Mem. Accad. Lincei 6, (1927) 602.

[78] T. Tietz, J. Chem. Phys. 25, (1956) 789.

[79] M. A. Kumakhov and F. F. Komarov, Energy Loss and Ion Ranges in Solids (Gordon and Breach, New York, 1981).

[80] M. Abramowitz and I. A. Stegun, Handbook of Mathematical Functions (Dover, New York, 1972).

[81] T. Tietz, J. Chem. Phys. 23, (1955) 1565; K. Umeda, J. Fac. Sci. Hokkaido Univ. Ser. IV, (1951) 1.

[82] J. F. Janni, At. Data Nucl. Data Tables 27, (1982) 341. 
[83] J. M. Anthony and W. A. Lanford, Phys. Rev. A 25, (1982) 1868.

[84] H. D. Betz, G. Hortig, E. Leischner, Ch. Schmelzer, B. Stadler and J. Weihrauch, Phys. Lett. 22, (1966) 643.

[85] P. Gombás, Die Statistische Theorie des Atom und ihre Anwendungen (Springer, Vienna, 1949); W. Lenz, Z. Phys. 77, 713 (1932) 713; H. Jensen, ibid. 77, (1932) 722 .

[86] J. H. Hubbell, W. J. Veigele, E. A. Briggs, R. T. Brown, D. T. Cromer and R. J. Howerton, J. Phys. Chem. Ref. Data 4, (1975) 471.

[87] L. C. Northcliffe and R. F. Schilling, At. Data Nucl. Data Tables 7, (1970) 233.

[88] D. C. Santry and R. D. Werner, Nucl. Instrum. and Methods B 1, (1984) 13.

[89] D. C. Santry and R. D. Werner, Nucl. Instrum. and Methods 178, (1980) 523.

[90] H. H. Andersen, J. F. Bak, H. Knudsen and B. R. Nielsen, Phys. Rev. A 16, (1977) 1929.

[91] H. H. Lin, L. W. Li, and E. Norbeck, Nucl. Instrum. and Methods B 17, (1986) 91.

[92] W. Booth and I. S. Grant, Nucl. Phys. 63, (1965) 481.

[93] O. B. Firsov, Sov. Phys. Zh. Eksp. Teor. Fiz., 36 (1959) 1517 [JETP 36, (1959) 1076].

[94] S. A. Cruz, C. Cisneros, and I. Alvarez, Phys. Rev. A 17, (1978) 132.

[95] W. Kuhn, Z. Physik 33 (1925) 408.

[96] F. Reiche y F. Thomas, Z. Physik 34, (1925) 510.

[97] E. H. Mortensen, J. Oddershede y J. R. Sabin, Nucl. Instr. and Meth. B69, (1992) 24.

[98] A. D. McLachlan y M. A. Ball, Rev. Mod. Phys. 36, (1964) 844. 
[99] R. A. Harris, J. Chem. Phys. 50, (1969) 3947.

[100] P. Jørgensen y J. Linderberg. Int. J. Quantum Chem. 4, (1970) 587.

[101] P. Jørgensen y J. Oddershede, J. Chem. Phys. 78, (1983) 1898.

[102] P. W. Atkins y R. S. Friedman, Molecular quantum mechanics, (Tercera edición. 1997, Oxford University press).

[103] Proceedings de el 172 seminario Heraeus sobre atomos y moleculas en campos externos fuertes, Bad Honnef, Alemania, 1997. Plenum, por ser publicado.

[104] Proceedings de el Taller de trabajo sobre Propiedades de moleculas en campos magneticos fuertes, Gainesville, Florida, 1996. Int. J. Quantum Chem. 64(5), 1997.

[105] P. Schmelcher, L. Cederbaum y U. Kappes, Molecules in magnetic fields: Fundamental aspects, en Conceptual Trends in Quantum Chemistry, paginas 1-51, Kluwer Academic Publishers, Dordrecht, 1994.

[106] L. I. Schiff, Quantum Mechanics, McGraw-Hill, 3era edición, 1968, pp. 521.

[107] J. Oddershede, Adv. Chem. Phys. 69, (1987) 201. 


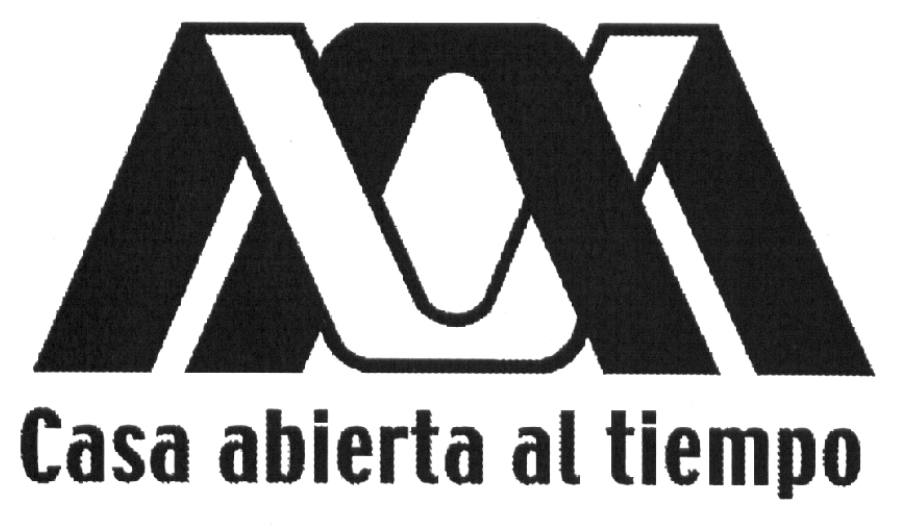

\section{Tratamiento teórico de efectos moleculares en el poder de frenamiento de iones en materia condensada}

Tesis que presenta:

M. en C. Remigio Cabrera Trujillo

para obtener el grado de Doctor en Ciencias

Departamento de Física,

División de Ciencias Básicas,

Universidad Autónoma Metropolitana-Iztapalapa,

México

Abril, 1998 